JOURNAL OF THE

AMERICAN MATHEMATICAL SOCIETY

Volume 22, Number 2, April 2009, Pages 467-519

S 0894-0347(08)00617-6

Article electronically published on September 17, 2008

\title{
CORPS DE NOMBRES PEU RAMIFIÉS ET FORMES AUTOMORPHES AUTODUALES
}

\author{
G. CHENEVIER ET L. CLOZEL
}

\section{INTRODUCTION}

Soient $S$ un ensemble fini de nombres premiers, $\mathbb{Q}_{S} \subset \overline{\mathbb{Q}}$ l'extension algébrique maximale de $\mathbb{Q}$ non ramifiée hors de $S$ (et l'infini) et $\mathrm{G}_{S}=\operatorname{Gal}\left(\mathbb{Q}_{S} / \mathbb{Q}\right)$. Un résultat bien connu de Minkowski affirme que si $S=\emptyset$, alors $\mathrm{G}_{S}=\{1\}$. En revanche, si $S$ est non vide, la structure de ces groupes $\mathrm{G}_{S}$ est très mal connue, et ce malgré leur omniprésence en géométrie arithmétique. Par exemple, un résultat d'Hermite assure que $G_{S}$ n'a qu'un nombre fini de sous-groupes fermés d'indice donné, mais on ne sait pour aucun $S \neq \emptyset$ si $\mathrm{G}_{S}$ est topologiquement engendré par un nombre fini d'éléments 11 Un autre problème du folklore consiste à déterminer les sousgroupes de décomposition de $\mathrm{G}_{S}$. Malgré le peu d'indices dont nous disposons pour appréhender cette question, il semble communément espéré que ces groupes sont aussi gros que la restriction imposée sur la ramification le permet : les résultats de cet article vont dans cette direction.

Plus précisément, supposons $S \neq \emptyset$ et fixons $p \in S$ un nombre premier. La donnée d'un plongement $\mathbb{Q}_{S} \longrightarrow \overline{\mathbb{Q}}_{p}$ definit un morphisme continu

$$
\operatorname{Gal}\left(\overline{\mathbb{Q}}_{p} / \mathbb{Q}_{p}\right) \longrightarrow \mathrm{G}_{S}
$$

dont la classe de conjugaison est indépendante du plongement choisi. Nous nous intéressons dans cet article à la question, soulevée notamment par R. Greenberg, de l'injectivité de ce morphisme ou, ce qui revient au même, à la densité de $\mathbb{Q}_{S}$ dans $\overline{\mathbb{Q}}_{p}$.

Ce problème a été récemment reconsidéré dans $\mathrm{Ch}$, auquel nous renvoyons le lecteur pour une discussion plus complète. Par exemple, il est démontré loc. cit. que (0.1) est injective dès que $S$ contient un nombre premier $\ell \neq p$ tel que $\ell \equiv 3 \bmod 4$ et que $-\ell$ est un carré modulo $p$. Après quelques réductions élémentaires, la preuve donnée loc. cit. consiste à démontrer l'existence de suffisamment de représentations automorphes (sur certains groupes unitaires) ayant des composantes locales inertielles partout prescrites, et de leur appliquer les travaux de Harris-Taylor HT] concernant les représentations galoisiennes associées (étendant des résultats antérieurs de Clozel et Kottwitz). Les corps de nombres obtenus sont alors ultimement extraits de l'action galoisienne sur la cohomologie $\ell$-adique de certains quotients

Received by the editors 28 juin 2007.

2000 Mathematics Subject Classification. Primary 11F70,11F72, 11F80.

Le deuxième auteur est un membre de l'Institut Universitaire de France.

${ }^{1}$ Ainsi que Serre nous l'a indiqué, cette question a été posée pour la première fois par Shafarevich au congrès international des mathématiciens de Stockholm (1962). Elle est brièvement discutée dans [NSW] chap. X, §2].

(C)2008 American Mathematical Society Reverts to public domain 28 years from publication 
arithmétiques « explicites 》 des espaces symétriques attachés aux groupes unitaires réels $\mathrm{U}(n, 1)(\mathbb{R})$, et ce pour tous les $n \geq 1$.

Notre objectif principal dans ce texte est de supprimer ces hypothèses parasites sur $\ell$, i.e. de démontrer le résultat suivant (Théorème 5.1).

Théorème A. Si $|S| \geq 2$, alors (0.1) est injective.

En particulier, pour tout entier $m \geq 1$, il existe un corps de nombres de degré multiple de $m$ et non ramifié hors de $S$.

Ce résultat avait été conjecturé dans [Ch], et ramené à des propriétés encore largement conjecturales de certaines formes modulaires de Siegel. La méthode employée ici est similaire, à ceci près que nous nous passons des groupes symplectiques et raisonnons directement sur le groupe linéaire $\mathrm{GL}_{2 n}$. En contrepartie, comme nous le verrons, les propriétés d'autodualité requises rendent les questions d'existence de représentations automorphes avec propriétés locales partout prescrites nettement plus subtiles et ardues.

Plus précisément, fixons $n \geq 1$ arbitraire, choisissons un $\ell \in S-\{p\}$ et fixons une composante de Bernstein $\mathfrak{c}_{p}$ du groupe $\mathrm{GL}_{2 n}\left(\mathbb{Q}_{p}\right)$. Nous voulons démontrer l'existence d'une représentation automorphe cuspidale $\Pi$ de $\mathrm{GL}_{2 n}(\mathbb{A})$ ayant les propriétés suivantes 2

(P1) $\Pi$ est autoduale, $\Pi_{\infty}$ est algébrique régulière, et $\Pi_{\ell}$ est essentiellement de carré intégrable (de sorte que $\Pi$ rentre dans le cadre des travaux de Harris et Taylor).

(P2) $\Pi$ est non ramifiée hors de $\{\infty, \ell, p\}$ et $\Pi_{p}$ est dans la composante $\mathfrak{c}_{p}$ fixée. Le choix de la représentation de carré intégrable $\Pi_{\ell}$ n'a pas d'importance pour notre application, et il nous sera en fait commode d'imposer que

(P3) $\Pi_{\ell}$ est la représentation de Steinberg.

Bien sûr, la composante $\mathfrak{c}_{p}$ ne peut être quelconque puisque $\Pi_{p}$ est nécessairement autoduale. Ce n'est en fait pas la seule obstruction, nous y reviendrons un peu plus loin. En ce qui concerne notre application au théorème plus haut, il nous suffira de considérer les composantes $\mathfrak{c}_{p}(\omega)$ induites, à partir du sous-groupe de Levi $\mathrm{GL}_{n}\left(\mathbb{Q}_{p}\right) \times \mathrm{GL}_{n}\left(\mathbb{Q}_{p}\right)$, d'une supercuspidale de la forme $\omega \times \check{\omega}$, où de plus $\omega$ n'a aucun twist non ramifié isomorphe à sa contragrédiente $\breve{\omega}$; mais nos résultats sont en fait de portée plus vaste. L'essentiel de nos efforts sera dirigé vers la preuve $\mathrm{du}$ résultat suivant (Théorème 3.2).

Théorème B. Pour tout $\omega$ comme ci-dessus et $\mathfrak{c}_{p}=\mathfrak{c}_{p}(\omega)$, il existe une représentation automorphe cuspidale $\Pi$ de $\mathrm{GL}_{2 n}(\mathbb{A})$ satisfaisant les propriétés (P1), (P2) et (P3).

Notre méthode pour construire la représentation $\Pi$ repose sur la formule des traces d'Arthur-Selberg pour le groupe $\mathrm{GL}_{2 n}$ tordu par l'automorphism 3

$$
\theta(g)={ }^{t} g^{-1} \text {. }
$$

La formule des traces est une identité de distributions $I_{\text {spec }}(\cdot)=I_{\text {geom }}(\cdot)$ dont l'utilisation pour ce type de problèmes est bien connue : appliquée à des fonctions tests

$$
f=\bigotimes_{v}^{\prime} f_{v}
$$

\footnotetext{
${ }^{2}$ Dans cet article, $\mathbb{A}=\mathbb{A}_{\mathbb{Q}}$ désignera les adèles de $\mathbb{Q}$.

${ }^{3}$ Pour des raisons techniques nous serons en fait amenés à considérer des variantes bénignes de cet automorphisme, nous négligeons cet aspect dans cette introduction.
} 
ne traçant que dans les données spectrales qui nous intéressent (ou presque), il s'agit de montrer la non nullité de son côté géométrique $I_{\text {geom }}(f)$.

Hors de $\infty, \ell, p, f_{v}$ sera pour nous simplement la fonction caractéristique de $\mathrm{GL}_{2 n}\left(\mathbb{Z}_{v}\right)$. En les places $\infty, \ell$ et $p$, les fonctions tests nécessaires seront des pseudocoefficients ou des fonctions de Bernstein bien choisies dont nous devrons maîtriser les intégrales orbitales $\theta$-tordues, et une partie du travail sera de les définir et d'établir certaines de leurs propriétés. Notons que bien que $\mathrm{GL}_{2 n}(\mathbb{R})$ n'ait pas de série discrète dès que $n>1$, il admet des séries $\theta$-discrètes, et ce sont des représentations de ce type qui nous intéressent à l'infini (en revanche, les composantes $\mathfrak{c}_{p}(\omega)$ décrites plus haut ne sont pas essentiellement discrètes, même parmi les composantes autoduales). Pour ces fonctions tests, une version simplifiée de la formule des traces due à Arthur [A2] s'applique, dont le côté géométrique se réduit aux termes portés par les élements $\theta$-semisimples et $\mathbb{Q}$-elliptiques.

Compte tenu de la rigidité de notre problème, l'unique liberté dont nous disposons est de faire varier $f_{\infty}$ parmi les pseudo-coefficients de séries $\theta$-discrètes cohomologiques. Ces représentations sont en fait naturellement paramétrées par le poids extrémal $\lambda$ d'une représentation irréductible $V_{\lambda}$ du groupe compact $\mathrm{SO}_{2 n+1}(\mathbb{R})$. Nous démontrerons alors ultimement que lorsque $\lambda$ tend vers l'infini en s'éloignant des murs, le côté géométrique de la formule des traces devient asymptotiquement équivalent à un terme unique que nous appelons le terme principal. Ce terme est (à un scalaire $>0$ près) l'intégrale orbitale tordue $\mathrm{TO}_{\gamma_{0}}(f)$ de $f$ en un certain élément $\theta$-semisimple $\mathbb{Q}$-elliptique

$$
\gamma_{0} \in \mathrm{GL}_{2 n}(\mathbb{Q})
$$

dont le centralisateur tordu est le groupe symplectique $\mathrm{Sp}_{2 n}$ sur $\mathbb{Q}$. Ainsi,

$$
I_{\text {spec }}(f)=I_{\text {geom }}(f) \underset{\lambda \rightarrow \infty}{\sim} \mathrm{TO}_{\gamma_{0}}(f)=C \cdot \operatorname{dim}\left(V_{\lambda}\right),
$$

où $C$ est une constante explicite non nulle ne dépendant que de $f^{\infty}$, et $f_{\infty}=f_{\infty, \lambda}$. Compte tenu de notre choix de $f^{\infty}$, le Théorème $\mathrm{B}$ est bien sûr conséquence de la formule (0.2) $4^{4}$

Pour démontrer ces résultats, nous devons surmonter un certain nombre de difficultés dont le traitement est réparti de la manière suivante. Le premier chapitre contient des préliminaires sur les caractères des représentations des groupes compacts connexes ainsi qu'une illustration de notre méthode dans un contexte non tordu. Les Chapitres 2, 3 et 4 sont consacrés au groupe $\mathrm{GL}_{2 n}$ tordu par l'automorphisme $\theta$ et à la preuve du Théorème B. Enfin, dans un dernier et court Chapitre 5 , nous montrons comment le Théorème $\mathrm{B}$ entraîne le Théorème $\mathrm{A}$; le lecteur peut commencer par celui-là en guise de motivation. Décrivons maintenant linéairement et plus en détail le contenu des Chapitres 1 à 4 .

Une idée sous-jacente à la méthode exposée ci-dessus (formule (0.2) ) est que la formule des traces se simplifie asymptotiquement « en faisant tendre le poids vers l'infini ». Cette idée se trouve déjà dans un argument de Serre [S2] dans le contexte du groupe $\mathrm{GL}_{2}$ non tordu. Dans le premier chapitre, nous l'étendons à son cadre général naturel. En guise d'application, nous établissons le résultat suivant

\footnotetext{
${ }^{4}$ En fait, une manière d'interpréter 0.2 est de la voir comme une formule de type RiemannRoch donnant asymptotiquement la dimension d'un certain espace de formes automorphes de poids variables et de niveau fixé (et engendrant des $\Pi$ satisfaisant (P1), (P2) et (P3)).
} 
(Théorème 1.3). Soit $G$ un $\mathbb{Q}$-groupe réductif connexe tel que $G(\mathbb{R})$ admet des séries discrètes, et dont le centre a la même composante déployée sur $\mathbb{R}$ et $\mathbb{Q}$.

Théorème C. Soient $\pi$ une représentation supercuspidale de $G\left(\mathbb{Q}_{p}\right)$ et $K$ un sousgroupe compact ouvert de $G\left(\mathbb{A}_{f}^{\{\infty, p\}}\right)$. Il existe une représentation automorphe cuspidale $\Pi$ de $G$ telle que $\Pi^{\{\infty, p\}}$ admet des $K$-invariants non nuls, $\Pi_{p}$ est isomorphe à une torsion non-ramifiée de $\pi$, et $\Pi_{\infty}$ est dans la série discrète.

La méthode employée est similaire à celle décrite plus haut à ceci près qu'elle utilise la formule des traces dans un cas non tordu, ce qui introduit un certain nombre de simplifications. Par exemple, les propriétés nécessaires des fonctions tests $f_{\infty}$ sont déjà connues. Nous avons besoin toutefois de démontrer le résultat suivant sur les caractères des groupes de Lie compacts connexes (Proposition 1.9). Soient $H$ un tel groupe compact et $T$ un tore maximal de $H$, notons $V_{\lambda}$ la representation irréductible de $H$ de poids extrémal $\lambda \in X^{*}(T)$. Si $\gamma \in H$ est non central, alors

$$
\operatorname{trace}\left(\gamma, V_{\lambda}\right) / \operatorname{dim}\left(V_{\lambda}\right) \longrightarrow 0
$$

lorsque $\lambda$ tend vers l'infini dans une direction convenable de $X^{*}(T) \otimes \mathbb{R}$.

Le Théorème $\mathrm{C}$ admet diverses variantes : nous pourrions par exemple demander de prescrire de plus les composantes de Bernstein $\mathfrak{c}_{v}$ de $\Pi_{v}$ en un ensemble fini fixé de places $v$ différentes de $\{p, \infty\}$, ce qui imposerait cependant en général quelques restrictions sur ces composantes $\mathfrak{c}_{v} 5$ Nous n'irons pas dans cette direction, notamment car nous le ferons plus loin dans le cadre de $\mathrm{GL}_{2 n}$ tordu. Le résultat ci-dessus et ses variantes n'entraînent pas le Théorème $\mathrm{B}$ car $\mathrm{GL}_{2 n}$ n'a pas les propriétés requises pour $n>1$. Cependant, nous pourrions l'appliquer au groupe orthogonal déployé $G=\mathrm{SO}_{2 n+1}^{*}$, de sorte que l'existence des représentations que nous recherchons découlerait en fait de la conjecture de transfert de $\mathrm{SO}_{2 n+1}^{*}$ vers $\mathrm{GL}_{2 n}$. Malheureusement, les cas actuellement connus de ce transfert nécessitent tous notamment une hypothèse de généricité de la représentation à transférer, et nous ne voyons pas comment assurer que nous construisons de telles représentations par la formule des traces. C'est pourquoi nous raisonnons par la suite directement sur le groupe $\mathrm{GL}_{2 n}$ tordu 6 par $\theta$.

Le Chapitre 2 contient le travail nécessaire à la place archimédienne. On y définit et étudie en détail les propriétés des fonctions $f_{\infty}$ dont nous avons besoin pour démontrer le Théorème $\mathrm{B}$ et dont nous avons déjà parlé plus haut. Si $\lambda$ est un poids extrémal d'une représentation irréductible $V_{\lambda}$ de $\mathrm{SO}_{2 n+1}(\mathbb{R})$, il lui correspond d'après Langlands une unique représentation $\theta$-discrète cohomologique $\pi_{\lambda}$ de $\mathrm{GL}_{2 n}(\mathbb{R})$ (et réciproquement). D'après une version du théorème de Paley-Wiener due à Mezo $[\mathrm{M}]$, cette représentation admet un pseudo-coefficient $f_{\infty, \lambda}$ dont la trace sur $\mathrm{GL}_{2 n}(\mathbb{R}) \theta$ isole $\pi_{\lambda}$ dans le spectre tempéré autodual de $\mathrm{GL}_{2 n}(\mathbb{R})$. Le résultat principal de ce chapitre est le suivant (Théorème 2.12).

Théorème D. Soit $\gamma \in \mathrm{GL}_{2 n}(\mathbb{R})$ un élément $\theta$-semisimple. Si $\gamma$ n'est pas $\theta$ elliptique, alors $\mathrm{TO}_{\gamma}\left(f_{\infty, \lambda}\right)=0$. Sinon, pour un choix convenable de mesure positive

\footnotetext{
${ }^{5}$ Un problème est que nous ne savons pas en général montrer que si une représentation automorphe cuspidale $\Pi$ de $G$ est telle que $\Pi_{\infty}$ est essentiellement discrète de paramètre suffisamment régulier, alors $\Pi$ est tempérée à toutes les places.

${ }^{6}$ Une autre méthode aurait consisté à construire des $\Pi$ comme plus haut qui sont génériques en produisant directement des séries de Poincaré (cf. [Sh2, §5]). Cependant, il semble alors plus délicat de prescrire $\Pi$ en toutes les places (plutôt que toutes sauf une), et nous n'avons pas poursuivi cette voie. En contrepartie, nous n'utilisons pas les résultats difficiles de transfert suscités.
} 
invariante sur la classe de $\theta$-conjugaison de $\gamma$, on a

$$
\operatorname{TO}_{\gamma}\left(f_{\infty, \lambda}\right)=e(\gamma, \lambda) \operatorname{trace}\left(\mathcal{N} \gamma, V_{\lambda}\right) \text {, }
$$

où $e(\gamma, \lambda)$ est un signe ne dépendant que de $\gamma$ et $\lambda$, et $\mathcal{N} \gamma \in \mathrm{SO}_{2 n+1}(\mathbb{R})$ est la "norme » de $\gamma$. En particulier, ces intégrales orbitales sont stables.

Pour l'étude de l'application norme dans ce contexte, nous renvoyons à un article de Waldspurger [W1. Les résultats de ce chapitre sont en fait plus complets. En utilisant des résultats de Bouaziz Bou, nous commençons par vérifier que le caractère tordu de $\pi_{\lambda}$ sur les éléments elliptiques fortement $\theta$-réguliers de $\mathrm{GL}_{2 n}(\mathbb{R})$ coïncide avec le caractère de $V_{\lambda}$ via l'application norme (pour une normalisation convenable de l'opérateur d'entrelacement, cf. Théorème 2.6). En particulier il est stable, ce qui est l'analogue d'un résultat de Waldspurger dans le cas p-adique W2. Ceci implique le Théorème D pour les intégrales orbitales tordues stables. Un argument simple ramène la forme précise du théorème au cas où $\pi_{\lambda}$ est à cohomologie non triviale pour le système de coefficients constant, cas où il a été démontré par Labesse [La1] 7

Le Chapitre 3 donne la preuve esquissée plus haut de la construction de la représentation $\Pi$. Dans un paragraphe, \$3.4 nous définissons et étudions la fonction $f_{\ell}$ dont nous avons besoin. C'est un pseudo-coefficient tordu de la représentation de Steinberg de $\mathrm{GL}_{2 n}\left(\mathbb{Q}_{\ell}\right)$ dont il nous faut calculer les intégrales orbitales tordues. Il n'est pas plus long ici de mener cette étude dans le cadre d'une groupe réductif connexe $G$ général, et d'un automorphisme $\theta$ d'ordre fini quelconque, et c'est le choix que nous adoptons. Nous imitons pour cela une méthode de Kottwitz K1 (voir aussi [BLS, §9])) consistant à réaliser géométriquement la fonction $f_{\ell}$ comme une fonction d'Euler-Poincaré pour l'automorphisme $\theta$ de $G$, et reposant ultimement sur les propriétés de l'immeuble de Bruhat-Tits de $G\left(\mathbb{Q}_{\ell}\right)$, des travaux de Serre sur les mesures d'Euler-Poincaré, et des résultats de Casselman et Borel-Wallach sur la cohomologie lisse de $G\left(\mathbb{Q}_{\ell}\right)$. À l'aide des résultats des Chapitres 1 et 2, nous démontrons la formule (0.2) conditionnellement au résultat suivant (Théorème 3.3) qui fera l'objet du Chapitre 4, et qui permet de montrer la non-annulation (cruciale) de la constante $C$.

Théorème E. Il existe une fonction lisse à support compact $f_{p}$ sur $\mathrm{GL}_{2 n}\left(\mathbb{Q}_{p}\right)$ dont les traces tordues sont nulles hors de la composante de Bernstein $\mathfrak{c}_{p}(\omega)$ et telle que

$$
\mathrm{TO}_{\gamma_{0}}\left(f_{p}\right) \neq 0 \text {. }
$$

Le rôle particulier joué par $\gamma_{0}$ résulte de ce que c'est l'unique classe de conjugaison $\mathbb{Q}$-elliptique $\theta$-semisimple de $\mathrm{GL}_{2 n}(\mathbb{Q})$ dont la norme est centrale (i.e. triviale) dans $\mathrm{SO}_{2 n+1}$. En particulier, cette classe cö̈ncide avec sa classe de conjugaison stable. En la place $\ell$, nous démontrons aussi un résultat analogue au Théorème E pour la fonction d'Euler-Poincaré $f_{\ell}$ (Proposition 3.8).

Le Chapitre 4 est voué à la preuve du Théorème E. Il s'agit du coeur technique de cet article. Avant d'en dire plus sur sa démonstration, il convient d'en discuter les tenants et les aboutissants. Oublions temporairement que $\mathfrak{c}_{p}$ a la forme nécessaire à notre application et supposons que c'est une composante autoduale quelconque. Comme nous l'avions sous-entendu plus haut, il y a une obstruction à ce que l'on puisse construire $\Pi$ avec $\Pi_{p}$ appartenant à $\mathfrak{c}_{p}$. En effet, comme on le verra au \2.1, il est nécessaire à cause du choix de $\Pi_{\infty}$ que le $L$-paramètre de $\Pi_{p}$ soit symplectique.

${ }^{7}$ Labesse nous a assuré qu'une rédaction ultérieure préciserait cette démonstration. 
Cela peut se déduire simplement des théorèmes de Harris-Taylor et Taylor-Yoshida. De même, le formalisme du groupe de Langlands suggère qu'une obstruction à inclure un ensemble fini quelconque de supercuspidales comme composantes locales d'une représentation automorphe cuspidale autoduale de $\mathrm{GL}_{2 n}(\mathbb{A})$ est qu'elles soient soit toutes symplectiques, soit toutes orthogonales (ceci avait déjà été observé par Prasad et Ramakrishnan dans [PR, §3], auquel les résultats de cet article apportent certaines réponses). D'une manière ou d'une autre, ce type d'hypothèse devra donc apparaître dans notre construction de $\Pi$. Du point de vue de notre méthode, cette obstruction se traduit exactement par la nullité ou non des intégrales orbitales tordues en l'élément $\gamma_{0}$ des fonctions de Bernstein tordues $f_{p}$ de $\mathfrak{c}_{p}$. Dans le cas où $\mathfrak{c}_{p}$ est la composante d'une supercuspidale $\pi$ autoduale, ceci est en parfait accord avec un résultat de Shahidi Sh2, Prop. 5.1] : il montre que cette intégrale orbitale est non nulle pour un coefficient de $\pi$ bien choisi si et seulement si le $L$-paramètre de $\Pi$ est symplectique 8 Ainsi, nous avons montré que l'analogue du Théorème $B$ vaut si l'on prend pour $\mathfrak{c}_{p}$ une telle composante avec $\pi$ symplectique. Le cas des composantes $\mathfrak{c}_{p}(\omega)$ nécessaires à notre application est étudié en détail dans ce chapitre et semble nouveau. Notons que les $L$-paramètres des représentations autoduales de $\mathfrak{c}_{p}(\omega)$ étant à la fois symplectiques et orthogonaux, nous nous attendons en fait à ce qu'il n'y ait pas d'obstruction dans ce cas.

Bien que le Théorème $\mathrm{E}$ soit de nature locale, notre démonstration utilise des arguments globaux. De façon naturelle d'après la théorie d'Arthur A4, l'alternative symplectique/orthogonale pour les représentations est étroitement liée à la stabilisation de la formule des traces, la partie «stable» provenant de $\mathrm{SO}(2 n+1)$ étant donnée par les représentations symplectiques. Pour certaines fonctions $f_{p}$ particulières déterminées par $\mathfrak{c}_{p}(\omega)$ (les «pseudo-coefficients positifs »), nous voulons démontrer la non-annulation de $\mathrm{TO}_{\gamma_{0}}$. Grâce à l'étude du caractère tordu des représentations de $\mathfrak{c}_{p}(\omega)$, on vérifie que les intégrales orbitales stables de $f_{p}$ ne sont pas identiquement nulles. Une version simplifiée et stabilisée de la formule des traces d'Arthur produit des $\Pi$ vérifiant les conditions précédentes mais présentant peut-être de la ramification parasite. Un argument nouveau de positivité (\$4.13), utilisant un lemme simple sur les caractères des groupes compacts (Prop. 4.15), nous permet alors de montrer la non-nullité du terme principal de $I_{\text {geom }}(f)$ et donc de $\mathrm{TO}_{\gamma_{0}}\left(f_{p}\right)$. Il est pour ceci crucial de normaliser partout l'opérateur d'entrelacement, associé à l'automorphisme $\theta$, de façon à fixer le vecteur de Whittaker. Les sorites nécessaires sont regroupés au paragraphe $\$ 4.7$.

Cet argument de positivité semble nouveau et susceptible d'autres applications ; nous en esquissons quelques-unes dans un dernier paragraphe (\$4.18) sur les propriétés locales-globales des représentations automorphes cuspidales autoduales de GL $(2 n)$. Nous montrons tout d'abord que les représentations automorphes étudiées par Clozel et Harris-Taylor sont toujours symplectiques (Théorème 4.20) :

Théorème $\mathbf{F}$ (Conditionnel). Soient $F$ un corps totalement réel et $\pi$ une représentation automorphe cuspidale de $\mathrm{GL}_{2 n}\left(\mathbb{A}_{F}\right)$. On suppose que $\pi$ est autoduale, essentiellement de carré intégrable en au moins une place finie, et cohomologique à toutes les places archimédiennes. Alors pour toute place $v$ de $F$, le L-paramètre de $\pi_{v}$ préserve une forme bilinéaire symplectique non dégénérée.

\footnotetext{
${ }^{8}$ Ainsi formulé, et ainsi qu'il est expliqué loc. cit., le résultat de Shahidi est conditionel au résultat suivant démontré ultérieurement par Henniart He2 Thm. 1.3] : $L\left(\pi, \Lambda^{2}, s\right)=$ $L\left(\Lambda^{2} \operatorname{rec}(\pi), s\right)$ où $\operatorname{rec}(\pi): \mathrm{W}_{\mathbb{Q}_{p}} \longrightarrow \mathrm{GL}_{2 n}(\mathbb{C})$ est le $L$-paramètre de $\pi$.
} 
En particulier, les représentations galoisiennes $\ell$-adiques associées sont aussi symplectiques. La démonstration du Théorème $F$ ne sera pas tout à fait complète car nous devrons admettre une propriété des intégrales orbitales des pseudo-coefficients du Théorème $D$, sur laquelle nous espérons revenir ultérieurement. Enfin, nous donnons des caractérisations des composantes locales essentiellement discrètes des représentations automorphes ci-dessus (Théorème 4.22), éclairant notamment certains aspects du spectre tempéré de GL $(2 n)$ tordu, sur un corps $p$-adique. Par exemple, nous obtenons le résultat suivant, précisant ceux de Shahidi discutés plus haut. Ici, $K$ est une extension finie de $\mathbb{Q}_{p}$.

Théorème G. Soit $\pi$ une représentation supercuspidale autoduale de $\mathrm{GL}_{2 n}(K)$ dont le L-paramètre est orthogonal. Alors les intégrales orbitales stables des pseudocoefficients tordus de $\pi$ sont toutes nulles.

Nous terminons en énonçant une conjecture sur la distribution de Plancherel sur le spectre tempéré autodual de $\mathrm{GL}_{2 n}(K)$ : elle est concentrée sur la variété des représentations symplectiques et c'est une mesure sur cette dernière (Conjecture 4.24).

Pour finir, notons qu'il est sans doute possible de raffiner notre méthode pour démontrer des versions plus fortes du Théorème B (par exemple : remplacer la composante $\mathfrak{c}_{p}(\omega)$ par une composante autoduale « symplectique » quelconque, demander que $\Pi_{\ell}$ soit non ramifiée, etc.). Cependant, même en admettant ces résultats, ainsi que des généralisations convenables des travaux de Harris-Taylor, nous ne voyons pas comment améliorer le Théorème A (i.e. autoriser $S=\{p\}$, cf. Ch, $\S 4.2])$.

Nos démonstrations reposent évidemment sur l'aride formule des traces tordue d'Arthur. De plus, comme on l'a dit, la forme précise des théorèmes est étroitement liée aux travaux annoncés par lui sur la fonctorialité entre groupes classiques et GL $(n)$. Certains de nos résultats, sans aucun doute, feront partie de l'exposé final de sa théorie.

Nous remercions Jean-Pierre Serre pour ses remarques, ainsi que le rapporteur pour sa lecture attentive qui nous a permis d'améliorer le texte.

\section{Existence de REPRÉSEntations En NIVEAU Minimal : CAS OÙ la CARACTÉristique D'Euler-PoinCARÉ EST NON Nulle}

1.1. Énoncé du résultat. Dans ce chapitre, et à titre de galop d'essai, nous démontrons le résultat naturel d'existence de représentations automorphes pour un groupe réductif $G$ sur $\mathbb{Q}$, vérifiant des conditions prescrites en deux places $\{\infty, p\}$, lorsque le groupe adjoint a une mesure d'Euler-Poincaré non nulle au sens de Serre [S1]. Soit donc $G$ un groupe réductif connexe défini sur $\mathbb{Q}$. Notons $Z$ le centre de $G, S$ le sous-tore maximal de $Z$ déployé sur $\mathbb{Q}$ ("composante déployée de $Z^{\prime \prime)}$.

Hypothèse 1.2. Les composantes déployées de $Z$ sur $\mathbb{Q}$ et $\mathbb{R}$ coïncident.

On a ainsi une suite exacte

$$
1 \longrightarrow S \longrightarrow Z \longrightarrow C \longrightarrow 1
$$

de $\mathbb{Q}$-groupes diagonalisables, la composante neutre de $C$ étant anisotrope sur $\mathbb{Q}$ et $\mathbb{R}$. On note

$$
A=S(\mathbb{R})^{+}
$$


(on désigne par ${ }^{+}$les composantes neutres topologiques).

On suppose enfin que la mesure d'Euler-Poincaré sur les quotients arithmétiques de $G(\mathbb{R}) / Z(\mathbb{R})$ - ou, ce qui revient au même, de $G(\mathbb{R}) / S(\mathbb{R})$ - est non nulle ; ceci revient à dire que $G(\mathbb{R}) / S(\mathbb{R})$ a une série discrète, ou qu'il admet une forme intérieure compacte.

Fixons un nombre premier $p$. Soit $D$ le plus grand tore déployé quotient de $G$ sur $\mathbb{Q}_{p}$ (noter que $D$ dépend de $p$ ). Soit $\pi_{p}$ une représentation supercuspidale de $G\left(\mathbb{Q}_{p}\right)$. L'orbite inertielle de $\pi_{p}$ est l'ensemble des représentations $\left\{\pi_{p} \otimes \chi\right\}$ où $\chi$ parcourt les caractères non-ramifiés de $D\left(\mathbb{Q}_{p}\right)$.

On fixe une mesure (finie) $G(\mathbb{A})$-invariante sur $A G(\mathbb{Q}) \backslash G(\mathbb{A})$ et on considère l'espace

$$
\mathcal{A}_{G}=L^{2}(A G(\mathbb{Q}) \backslash G(\mathbb{A}))
$$

muni de la représentation naturelle de $G(\mathbb{A})$. Une représentation cuspidale de $G(\mathbb{A})$ sera, par définition, une représentation irréductible apparaissant dans le sous-espace des fonctions cuspidales (au sens usuel) de $\mathcal{A}_{G}$. Si $K \subset G\left(\mathbb{A}_{f}^{p}\right)$ est un sous-groupe compact ouvert, elle est de niveau $K$ si son espace des $K$-invariants est non nul.

Théorème 1.3. Soit $\pi_{p}$ une représentation cuspidale de $G\left(\mathbb{Q}_{p}\right)$, et $K \subset G\left(\mathbb{A}_{f}^{p}\right)$. Il existe une représentation cuspidale $\Pi=\bigotimes_{v} \Pi_{v}$ de $G(\mathbb{A})$, de niveau $K$, telle que

(i) $\Pi_{\infty}$ est une représentation de la série discrète de $G(\mathbb{R}) / A$,

(ii) $\Pi_{p}$ appartient à l'orbite inertielle de $\pi_{p}$.

Remarque 1.4. La démonstration se simplifie quand $G$ est semisimple. Nous n'avons pas voulu faire cette hypothèse car les groupes apparaissant dans les applications naturelles des formes automorphes à l'arithmétique (cf. [HT] ) sont rarement semisimples. Ces questions de passage d'un groupe à un groupe isogène (au centre près) recèlent des phénomènes non-triviaux.

1.5. Fonctions locales : Cas réel. Nous décrivons des fonctions particulières sur $G(\mathbb{R})$ et $G\left(\mathbb{Q}_{p}\right)$ adaptées à notre problème.

Considérons d'abord la place archimédienne. Puisque $G(\mathbb{R}) / A$ a une série discrète, $G$ a une forme intérieure réelle $G^{*}$ anisotrope modulo le centre. On dira qu'une représentation de $G(\mathbb{R})$ ou $G^{*}(\mathbb{R})$ est dans la série discrète si elle est unitaire et de carré intégrable modulo le centre. D'après Langlands et Shelstad [She, il y a une bijection entre représentations unitaires irréductibles de $G^{*}(\mathbb{R})$ (dont on notera $\widehat{G^{*}(\mathbb{R})}$ l'ensemble) et $L$-paquets de séries discrètes de $G(\mathbb{R})$. Pour $\delta \in \widehat{G^{*}(\mathbb{R})}$, soit $\Pi(\delta)$ le $L$-paquet associé. Le caractère central $\omega$ de toute représentation $\pi \in \Pi(\delta)$ coïncide avec celui de $\delta$. Nous nous intéressons aux représentations telles que $\left.\omega\right|_{A}=$ 1.

Notons $\bar{G}$ le groupe $G(\mathbb{R}) / A$. D'après Clozel-Delorme CloD et Labesse La2, il existe, pour tout $\pi \in \Pi(\delta)$, une fonction $f_{\pi} \in C_{c}^{\infty}(\bar{G})$ (d'ailleurs $K_{\infty}$-finie pour un sous-groupe compact maximal $K_{\infty}$ de $\left.G(\mathbb{R})\right)$ telle que

$$
\left\langle\operatorname{trace} \pi, f_{\pi}\right\rangle=1 \text {, }
$$

la trace de $f_{\pi}$ dans toute autre représentation tempérée irréductible de $\bar{G}$ étant nulle. Il en résulte que

$$
f_{\pi}(z g)=\omega(z)^{-1} f_{\pi}(g)
$$

si $g \in \bar{G}$ et $z$ appartient au centre $\bar{Z}$ de $\bar{G}$. La formule (1.4) suppose choisie une mesure de Haar $d \bar{g}$ sur $\bar{G}$. 
Soit $h$ une fonction $C^{\infty}$ à support compact sur $A ; G(\mathbb{R})$ est isomorphe à $A \times \bar{G}$ et les fonctions $h \otimes f_{\pi}$ sont donc des fonctions sur $G(\mathbb{R})$; si $\bar{f}_{\delta}^{*}$ est un coefficient de $\delta\left(\operatorname{avec} \operatorname{trace} \delta\left(\bar{f}_{\delta}^{*}\right) \neq 0\right)$, alors

$$
h \otimes \bar{f}_{\delta}^{*}=: f_{\delta}^{*}
$$

est une fonction sur $G^{*}(\mathbb{R})$. Les fonctions $f_{\delta}=\sum_{\pi \in \Pi} h \otimes f_{\pi}$ et $f_{\delta}^{*}$, sur $G(\mathbb{R})$ et $G^{*}(\mathbb{R})$, sont associées au sens de Shelstad et de l'appendice de [CloD]. Il en résulte que l'on a les propriétés suivantes. Soit $\gamma \in G(\mathbb{R})$ un élément semisimple, $I \subset G$ son centralisateur; pour $f \in \mathcal{C}_{c}^{\infty}(G(\mathbb{R}))$ considérons

$$
\mathrm{O}_{\gamma}(f)=\int_{I(\mathbb{R}) \backslash G(\mathbb{R})} f\left(g^{-1} \gamma g\right) \frac{d g}{d i}
$$

où $d g, d i$ sont des mesures de Haar. Alors :

Lemme 1.6. (i) Si $\gamma$ n'est pas $\mathbb{R}$-elliptique, $O_{\gamma}\left(f_{\delta}\right)=0$.

(ii) Si $\gamma$ est $\mathbb{R}$-elliptique, associé à un élément $\gamma^{*}$ de $G^{*}(\mathbb{R})$,

$$
O_{\gamma}\left(f_{\delta}\right)=e(\gamma) O_{\gamma^{*}}\left(f_{\delta}^{*}\right)
$$

pour une normalisation convenable des mesures sur les centralisateurs $I(\mathbb{R})$ et $I^{*}(\mathbb{R})$, et où $e(\gamma)= \pm 1$ ne dépend que de $\gamma$.

(iii) En particulier, pour $\gamma \mathbb{R}$-elliptique,

$$
O_{\gamma}\left(f_{\delta}\right)=e(\gamma) h(\alpha) \theta_{\delta}\left(\bar{\gamma}^{*-1}\right)
$$

où $\gamma=\alpha \bar{\gamma}$ selon l'isomorphisme choisi entre $G(\mathbb{R})$ et $A \times \bar{G}$, et où $\bar{\gamma}^{*}$ est l'image de $\gamma^{*}$ dans $G^{*}(\mathbb{R}) / A$, $\theta_{\delta}$ étant le caractère de la représentation $\delta$.

Preuve. La partie (i) est bien connue et résulte des propriétés des fonctions $f_{\pi}$. La partie (ii) est due à Shelstad [She. (Nous ne décrirons pas les normalisations des mesures, pour nous inessentielles.) Le signe $e(\gamma)$ est décrit par Shelstad. Si $\gamma$ est central, $e(\gamma)=1$. Enfin (iii) résulte simplement des relations d'orthogonalité de Schur sur $\bar{G}$.

Noter que quand $\delta$ varie, le support des fonctions $f_{\pi}$ (et donc $f_{\delta}$ ) peut être choisi contenu dans un compact fixe. Ceci résulte de CloD], ou d'ailleurs de l'argument de Labesse [La2]. Nous devrons enfin contrôler la variation avec $\delta$ de $\theta_{\delta}(\bar{\gamma})$. Noter que l'on peut évidemment faire l'hypothèse suivante :

Hypothèse 1.7. $S$ ne contient aucun sous-tore $S^{\prime} \neq 1$ qui soit facteur direct dans $G$.

Il en fait équivalent de demander qu'un sous-tore (automatiquement rationnel) $S^{\prime} \subset S$ soit facteur direct dans $G$ sur $\mathbb{R}$ et sur $\mathbb{Q}$. En effet, le cocentre $G / G_{\text {der }}$ étant $\mathbb{Q}$-isogène à $Z$, ses composantes déployées sur $\mathbb{R}$ et sur $\mathbb{Q}$ coïncident, de sorte que tout caractère réel $G \rightarrow \mathbb{G}_{m}$ est défini sur $\mathbb{Q}$. Enfin, comme le centre et le cocentre de deux formes intérieures sont canoniquement isomorphes, l'hypothèse 1.7 est encore équivalente à demander que $G^{*}$ n'ait aucun tore central déployé qui soit facteur direct sur $\mathbb{R}$.

Lemme 1.8. Sous l'hypothèse $\left[1.7, G^{*}(\mathbb{R})\right.$ est connexe, et $G^{*}(\mathbb{R}) / A$ est donc compact connexe. 
Preuve. Il existe un sous-groupe algébrique $H \subset G^{*}$ défini sur $\mathbb{R}$ tel que $H(\mathbb{R})$ soit un sous-groupe compact connexe maximal de $G^{*}(\mathbb{R})$. Les algèbres de Lie de $S$ et de $H$ sont alors en somme directe dans $\operatorname{Lie}\left(G^{*}\right)$, de sorte que le $\mathbb{R}$-morphisme naturel $S \times H \longrightarrow G^{*}$ est surjectif (sur les $\mathbb{C}$-points), de noyau $\mu:=S \cap H$ fini, i.e.

$$
1 \longrightarrow \mu \longrightarrow(S \times H) \longrightarrow G^{*} \longrightarrow 1 .
$$

En fait, la suite ci-dessus reste exacte après passage aux $\mathbb{R}$-points, i.e. $G^{*}(\mathbb{R})=$ $H(\mathbb{R}) \cdot S(\mathbb{R})$. En effet, $G^{*} / S$ est réductif connexe et par construction $\left(G^{*} / S\right)(\mathbb{R})=$ $G^{*}(\mathbb{R}) / S(\mathbb{R})$ est compact : ce dernier est donc aussi connexe. Cela conclut car $H(\mathbb{R})$ est connexe et $\operatorname{Lie}(H(\mathbb{R})) \rightarrow \operatorname{Lie}\left(\left(G^{*} / S\right)(\mathbb{R})\right)$ est un isomorphisme.

Le tore $S$ étant déployé, $S(\mathbb{R})=A \times\{ \pm 1\}^{\operatorname{dim}(S)}$ et $\mu \subset\{ \pm 1\}^{\operatorname{dim}(S)}$. L'exactitude de (1.5) sur les $\mathbb{R}$-points assure que

$$
G^{*}(\mathbb{R}) \simeq A \times\{ \pm 1\}^{\operatorname{dim}(S)} / \mu \times H(\mathbb{R}) .
$$

Mais si $\mu \subsetneq\{ \pm 1\}^{\operatorname{dim}(S)}$, alors $S$ admet un sous-tore strict (necéssairement facteur direct sur $\mathbb{R}$ ) contenant $\mu$, de sorte que la suite exacte (1.5) contredit l'hypothèse 1.7. ce qui conclut.

Soit alors $\bar{T}$ un tore maximal de $\bar{G}^{*}=G^{*}(\mathbb{R}) / A$. On peut paramétrer les représentations $\delta$ par leur plus haut poids $\lambda \in X=X^{*}(\bar{T})$. Si $\bar{\gamma}$ appartient au centre $\bar{Z}$ de $\bar{G}^{*}$, on a

$$
\theta_{\delta}(\bar{\gamma})=\operatorname{deg}(\delta) \omega(\bar{\gamma}) .
$$

Notons $\delta(\lambda)$ la représentation associée à $\lambda \in X$. Alors $\operatorname{deg} \delta(\lambda)$ est donné par le polynôme de Weyl,

$$
P(\lambda)=\prod_{\alpha} \frac{\langle\alpha, \lambda+\rho\rangle}{\langle\alpha, \rho\rangle},
$$

les produits portant sur un ensemble de racines positives dont $\rho$ est la demi-somme.

La proposition qui suit sera appliquée à $\bar{G}^{*}$ mais est vraie pour tout groupe de Lie compact connexe. Jusqu'à la fin du $\$ 1.5, G$ désignera un tel groupe, $T$ un tore maximal de $G$ et $X$ le groupe des caractères de $T$. On fixe un ensemble de racines positives pour $(G, T)$. Pour $\lambda \in X$ dominant, soit $\theta_{\lambda}$ le caractère de la représentation de plus haut poids $\lambda$ de $G$.

Proposition 1.9. Soit $\gamma \in T \subset G$. Pour $\lambda$ dominant,

$$
\theta_{\lambda}(\gamma)=\sum_{i} E_{i}(\gamma, \lambda) P_{i}(\lambda) .
$$

La somme est finie; les $E_{i}(\gamma, \lambda)$ sont des fractions rationnelles (dont les degrés dépendent de $\lambda$ ) en les $\gamma^{\chi}$, où $\chi$ parcourt une base de $X$, dont les dénominateurs sont non nuls en $\gamma$ et indépendants de $\lambda ; E_{i}(\gamma, \lambda)$ est uniformément bornée quand $\lambda$ varie. De plus, $P_{i}(\lambda)$ est un polynôme de degré strictement inférieur à celui de $P(\lambda)$ si $\gamma$ n'est pas central.

Preuve. Si $\gamma$ est régulier ou central, la proposition résulte de la formule du caractère ou du degré de Weyl. Si le centralisateur de $\gamma$ est un sous-groupe de Levi, elle se déduit de la formule de Kostant. En général, nous imitons l'une des démonstrations de celle-ci. 
Soit $G_{\text {der }}$ le groupe dérivé de $G$. On vérifie aussitôt qu'il existe un revêtement connexe fini $\widetilde{G}$ de $G$ dont le groupe dérivé est simplement connexe, et que la proposition pour $G$ résult 9 de celle pour $\widetilde{G}$. On suppose donc $G_{\text {der }}$ simplement connexe ; le centralisateur de tout élément $\gamma$ est alors connexe.

Fixons un tel élément $\gamma$. Notons $M$ le centralisateur de $\gamma$ dans $G$, soit $R(G, T)$ et $R(M, T)$ les ensembles de racines associés, $R_{+} \subset R$ les racines positives, $\Delta \subset R_{+}$ les bases, pour $G$ et $M, \rho, \rho_{M}$ les demi-sommes de racines associées. On suppose que $R_{+}(M, T)=R_{+}(G, T) \cap R(M, T)$; quitte à prendre un nouveau revêtement on peut supposer que $\rho$ et $\rho_{M}$ appartiennent à $X$. Soit $W$ le groupe de Weyl de $(G, T)$, $W_{M}$ celui de $(M, T)$. Soit

$$
W^{M}=\left\{w \in W: w^{-1} \alpha \in R_{+}(G, T) \forall \alpha \in \Delta_{M}\right\} .
$$

Comme dans le cas parabolique :

Lemme 1.10. Si $w \in W, w$ s'écrit de manière unique $w=w_{s} w_{u}$ avec $w_{s} \in W_{M}$ et $w_{u} \in W^{M}$.

Preuve. Soient en effet $C_{G}^{+}, C_{M}^{+}$les intérieurs des chambres de Weyl positives, dans $X \otimes \mathbb{R}$, relatives à $G$ et $M$. Fixons $H \in C_{G}^{+}$. Alors $w \in W$ appartient à $W^{M}$ si et seulement si

$$
\langle\alpha, w(H)\rangle>0 \quad\left(\alpha \in \Delta_{M}\right),
$$

c'est-à-dire si $w(H) \in C_{M}^{+}$.

Soit $w \in W$; il existe alors $w_{s} \in W_{M}$ tel que $w_{s}^{-1} w(H) \in C_{M}^{+}$. Donc $w_{s}^{-1} w \in$ $W^{M}$, d'où la décomposition cherchée. Si $w=w_{s} w_{u}=w_{s}^{\prime} w_{u}^{\prime}, w_{u}(H)$ et $w_{u}^{\prime}(H)$ sont dans $C_{M}^{+}$et $w_{s} C_{M}^{+}$rencontre $w_{s}^{\prime} C_{M}^{+}$, donc $w_{s}=w_{s}^{\prime}$.

Lemme 1.11. Si $\lambda \in X$ est dominant pour $G$ et $w_{u} \in W^{M}$, alors $w_{u}(\lambda+\rho)-\rho_{M}$ est dominant pour $M$.

Preuve. Il faut en effet vérifier que

$$
2 \frac{\left\langle w_{u}(\lambda+\rho), \alpha\right\rangle}{\langle\alpha, \alpha\rangle}-2 \frac{\left\langle\rho_{M}, \alpha\right\rangle}{\langle\alpha, \alpha\rangle} \geq 0 \quad\left(\alpha \in \Delta_{M}\right) .
$$

Le premier terme est entier, positif car $w_{u}^{-1} \alpha \in R^{+}(G, T)$, strictement positif car $\lambda+\rho$ est régulier. Le second est égal à 1 .

Démontrons alors la proposition. Ecrivons d'abord, formellement (= dans le groupe de Grothendieck de $T$ ) :

où

$$
\theta_{\lambda}=\frac{N}{D}
$$

$$
\begin{aligned}
& N=\sum_{w \in W} \varepsilon(w) e^{w(\lambda+\rho)}, \\
& D=\sum_{w \in W} \varepsilon(w) e^{w \rho}
\end{aligned}
$$

${ }^{9}$ Remarquer notamment que l'image inverse du centre de $G$ est le centre de $\widetilde{G}$. 
$\varepsilon$ étant le signe sur $W$. Alors

$$
\begin{aligned}
N & =\sum_{w_{u} \in W^{M}} \varepsilon\left(w_{u}\right) \sum_{w_{s} \in W_{M}} \varepsilon\left(w_{s}\right) e^{w_{s} w_{u}(\lambda+\rho)} \\
& =\sum_{w_{u} \in W^{M}} \varepsilon\left(w_{u}\right) \sum_{w_{s} \in W_{M}} \varepsilon\left(w_{s}\right) e^{w_{s}\left(\lambda_{u}+\rho_{M}\right)}
\end{aligned}
$$

où l'on a écrit $w_{u}(\lambda+\rho)=\lambda_{u}+\rho_{M}$ d'après le Lemme 1.11 avec $\lambda_{u}$ dominant pour $M$. Chacun des termes de la somme ci-dessus indexé par $w_{u}$ est donc un numérateur de Weyl pour $M$. Par ailleurs, si l'on pose $S(M)=R^{+}(G, T) \backslash R^{+}(M, T)$, alors

$$
\begin{aligned}
D & =e^{\rho} \prod_{\alpha \in R^{+}(G, T)}\left(1-e^{-\alpha}\right) \\
& =D_{M} e^{\rho-\rho_{M}} \prod_{\alpha \in S(M)}\left(1-e^{-\alpha}\right) .
\end{aligned}
$$

Ainsi

$$
\frac{N}{D}=e^{\rho_{M}-\rho} \prod_{\alpha \in S(M)}\left(1-e^{-\alpha}\right)^{-1}\left(\sum_{w_{u}} \varepsilon\left(w_{u}\right) \theta_{M, \lambda_{u}}\right)
$$

où $\theta_{M, \lambda_{u}}$ est le caractère de $M$ associé à $\lambda_{u}$. Cette expression donne la valeur de $\theta_{\lambda}$ pour un élément $G$-régulier $t \in T$. Si $t \longrightarrow \gamma$, les termes $\left(1-t^{-\alpha}\right)$ pour $\alpha \notin R(M, T)$ restent non nuls; $\theta_{M, \lambda_{u}}$ a pour limite $\omega_{\lambda_{u}}(\gamma) P_{M}\left(\lambda_{u}\right)$, où $\omega_{\lambda_{u}}(\gamma)=\gamma^{w_{u}(\lambda+\rho)-\rho_{M}}$ et $P_{M}$ est le polynôme de Weyl pour $M$. Ainsi

$$
\theta_{\lambda}(\gamma)=\frac{\gamma^{\rho_{M}-\rho}}{\Pi_{\alpha \in S(M)}\left(1-\gamma^{-\alpha}\right)} \sum_{w_{u} \in W^{M}} \varepsilon\left(w_{u}\right) \gamma^{w_{u}(\lambda+\rho)-\rho_{M}} P_{M}\left(\lambda_{u}\right),
$$

ce qui démontre la proposition. L'assertion sur le degré des $P_{i}$ résulte des formules du degré de Weyl pour $G$ et $M$ et de ce que $S(M) \neq \emptyset$ si et seulement si $\gamma$ n'est pas central.

Corollaire 1.12. Si $\gamma \in G$ n'est pas central, alors $\frac{\theta_{\lambda}(\gamma)}{P(\lambda)}$ tends vers 0 lorsque $\lambda \in X^{*}(T) \otimes \mathbb{R}$ tend vers l'infini en s'éloignant des murs des chambres de Weyl.10

Preuve. On peut supposer $\gamma \in T$. Dans les notations précédentes, nous avons explicitement

$$
\frac{P_{M}\left(\lambda_{u}\right)}{P(\lambda)}=\frac{\prod_{\alpha \in R^{+}(G, T)}\langle\rho, \alpha\rangle}{\prod_{\alpha \in R^{+}(M, T)}\left\langle\rho_{M}, \alpha\right\rangle}\left(\prod_{\alpha \in R^{+}(G, T) \backslash w_{u}^{-1} R^{+}(M, T)}\langle\lambda+\rho, \alpha\rangle\right)^{-1} .
$$

\footnotetext{
${ }^{10}$ Nous entendons par là que pour tout $\alpha \in R^{+}(G, T),\langle\lambda, \alpha\rangle$ tend vers l'infini.
} 
1.13. Fonctions locales en $p$. L'ensemble des caractères non ramifiés $\chi$ de $D\left(\mathbb{Q}_{p}\right)$ forme un tore complexe; soit $F_{p}(\chi)$ une fonction polynomiale sur celui-ci et qui de plus est invariante par le sous-groupe (fini) des $\chi$ tels que $\pi_{p} \otimes \chi \simeq \pi_{p}$. Par la théorie de Bernstein, on sait alors qu'il existe une fonction $f_{p}$ sur $G\left(\mathbb{Q}_{p}\right)$ ne traçant de manière non nulle que dans l'orbite inertielle de $\pi_{p}$, et telle que

$$
\left\langle\operatorname{trace}\left(\pi_{p} \otimes \chi\right), f_{p}\right\rangle=F_{p}(\chi) .
$$

La formule de Plancherel montre que $f_{p}(1)=1$ pour un choix convenable de $F_{p}$.

Soit par ailleurs $S\left(\mathbb{Q}_{p}\right) \subset G\left(\mathbb{Q}_{p}\right)$ (\$1.1) et soit $\varepsilon$ un élément de $\{ \pm 1\}^{\operatorname{dim} S}$, plongé dans $S\left(\mathbb{Q}_{p}\right)$. Puisque $\chi(\varepsilon)=1$, la formule de Plancherel implique que

$$
f_{p}(\varepsilon)=\omega_{p}(\varepsilon)^{-1}
$$

où $\omega_{p}$ est le caractère central de $\pi_{p}$.

1.14. Démonstration du Théorème 1.3. Notons $R$ la représentation de $G(\mathbb{A})$ dans la partie cuspidale de $\mathcal{A}_{G}$. Soit $f$ la fonction sur $G(\mathbb{A})$ donnée par

$$
f=f_{\infty} \otimes f_{p} \otimes f^{p, \infty}
$$

où $f_{\infty}=f_{\delta}$ et $f_{p}$ viennent d'être définies, et $f^{p, \infty}$ est la fonction caractéristique de $K$. Puisque $f_{p}$ est cuspidale, et que les intégrales orbitales de $f_{\infty}$ en les éléments non elliptiques s'annulent, la formule des traces simplifiée de Deligne et Kazhdan (cf. Henniart [He1]) s'applique :

$$
\operatorname{trace} R(f)=\sum_{\gamma} v(\gamma) O_{\gamma}\left(f_{\infty}\right) O_{\gamma}\left(f_{p}\right) O_{\gamma}\left(f^{p, \infty}\right)
$$

où $\gamma$ parcourt les classes de conjugaison elliptiques de $G(\mathbb{Q}$ ) (en fait $\mathbb{R}$-elliptiques vu les propriétés de $f_{\infty}$ ) et $v(\gamma)>0$ est un volume. Puisque les supports de nos fonctions sont contenus dans un compact fixe, cette somme est finie, uniformément quand $f_{\infty}$ varie (on pourrait aussi, bien sûr, déduire cette identité des résultats d'Arthur A2]).

Pour $f_{\infty}=f_{\delta}$ et $\gamma$ central, $O_{\gamma}\left(f_{\infty}\right)=f_{\infty}(\gamma)$ est essentiellement égale au polynôme de Weyl en le paramètre $\lambda$ de $\delta$. Considérons une suite de $\lambda \in X^{*}(\bar{T})$ tendant vers l'infini dans une direction régulière. Si $\gamma$ n'est pas central, $O_{\gamma}\left(f_{\delta}\right)$ est négligeable par rapport à $P(\lambda)$ d'après le Lemme 1.6 et le Corollaire 1.12. On en déduit que

$$
P(\lambda)^{-1} \operatorname{trace} R(f)=v \sum_{\gamma \in Z(\mathbb{Q})} h(\alpha) \omega_{\lambda}^{-1}(\bar{\gamma}) f_{p}(\gamma) f^{p, \infty}(\gamma)+o(1),
$$

où l'on a utilisé les notations du Lemme 1.6] : $\gamma=\alpha \bar{\gamma}, \alpha \in A, \bar{\gamma} \in G(\mathbb{R}) / A$. La fonction $h \in \mathrm{C}_{c}^{\infty}(A)$ est pour l'instant arbitraire.

Utilisons la suite exacte (1.3), qui reste exacte après passage aux points rationnels. Si $F$ est la fonction de $\gamma$ figurant dans la somme (1.7), on considère donc

$$
\sum_{c \in C(\mathbb{Q})} \sum_{s \in S(\mathbb{Q})} F(s \gamma)
$$

où l'on a choisi un représentant $\gamma \in Z(\mathbb{Q})$ de $c$. Écrivons $s=s^{+} \varepsilon$, où $\varepsilon \in \mathcal{E}:=$ $\{ \pm 1\}^{\operatorname{dim}(S)} \subset S(\mathbb{Q})$ et $s^{+} \in S(\mathbb{Q}) \cap A$. Si $\gamma=\alpha \bar{\gamma}$,

$$
f_{\infty}(s \gamma)=h\left(s^{+} \alpha\right) \omega_{\lambda}^{-1}(\overline{\varepsilon \gamma}) .
$$


La somme étant finie, on peut supposer que les seuls termes présents vérifient $s^{+} \alpha=1$ en prenant le support de $h$ suffisamment proche de 1 ; quitte à changer le représentant $\gamma$ on peut donc supposer $\alpha=1$ et donc $s^{+}=1$ pour tous les termes de (1.8), qui s'écrit alors

$$
\sum_{c \in C(\mathbb{Q})} \sum_{\varepsilon \in \mathcal{E}} \omega_{\lambda}^{-1}(\varepsilon \bar{\gamma}) f_{p}(\varepsilon \gamma) f^{p, \infty}(\varepsilon \gamma)
$$

Si $c=1$, le terme correspondant est

$$
\sum_{\varepsilon} \omega_{\lambda}^{-1}(\varepsilon) f_{p}(\varepsilon) f^{p, \infty}(\varepsilon)
$$

Puisque $f_{p}(\varepsilon)=\omega_{p}^{-1}(\varepsilon)$, il est égal à

$$
\sum_{\varepsilon} f^{p, \infty}(\varepsilon) \geq f^{p, \infty}(1)>0
$$

si $\left.\omega_{\lambda}\right|_{\mathcal{E}}=\left.\omega_{p}^{-1}\right|_{\mathcal{E}}$. Fixons $\lambda_{0}$ vérifiant cette condition. Soit $\lambda=\lambda_{0}+\mu$, avec $\mu \in X$ trivial sur $\mathcal{E} \subset \bar{G}^{*}$. Soit $\bar{Z}$ le centre de $\bar{G}^{*} ;$ donc $\bar{Z}=Z(\mathbb{R}) / A$. La suite exacte

$$
1 \longrightarrow S(\mathbb{R}) \longrightarrow Z(\mathbb{R}) \longrightarrow C(\mathbb{R}) \longrightarrow 1
$$

implique que $\bar{Z} / \mathcal{E}=C(\mathbb{R})$. Pour de tels $\lambda$, (1.9) se réécrit

$$
\sum_{c \in C(\mathbb{Q})} a(c) \mu^{-1}(c)
$$

où l'on a identifié dans l'écriture $\mu(c)$ l'élément $c \in C(\mathbb{Q})$ à l'image de $\bar{\gamma} \in \bar{Z}$ dans $C(\mathbb{R})$. De plus $a(1) \neq 0$ et $a(c)$ est à support finie. Puisque, tout en faisant tendre $\lambda$ vers l'infini dans les directions semisimples de $\bar{G}^{*}$, on peut prendre pour $\mu_{\mid C(\mathbb{R})}$ des caractères arbitraires, la théorie de Fourier sur ce tore compact implique que (1.10) prend des valeurs non nulles.

D'après (1.7), on en déduit que pour $\lambda$ assez grand et soumis aux conditions spécifiées ci-dessus,

$$
P(\lambda)^{-1} \operatorname{trace} R(f) \neq 0 \text {. }
$$

Mais

$$
\operatorname{trace} R(f)=\sum_{\Pi \text { cuspidale }} \operatorname{trace} \Pi_{\infty}\left(f_{\infty}\right) \operatorname{trace} \Pi_{p}\left(f_{p}\right) \operatorname{dim}\left(\pi^{p, \infty}\right)^{K_{p, \infty}},
$$

avec trace $\Pi_{\infty}\left(f_{\infty}\right)=\left(\int_{A} h(a) d a\right)$ trace $\bar{\Pi}_{\infty}\left(f_{\delta}\right)$, où $\bar{\Pi}_{\infty}$ est la restriction de $\Pi_{\infty}$ à $\bar{G}$. Si le paramètre $\lambda$ est assez régulier, toute représentation de $\bar{G}$ vérifiant trace $\bar{\Pi}_{\infty}\left(f_{\delta}\right) \neq 0$ appartient au $L$-paquet de séries discrètes $\left.\Pi(\delta)(\underline{V}]\right)$. Ceci termine la démonstration.

Remarque 1.15. Comme on l'a indiqué, le recours à la formule des traces simplifiée n'est pas nécessaire. En particulier, on aurait pu considérer une représentation $\pi_{p}$ appartenant à la série discrète, pour laquelle on peut construire une fonction $f_{p}$ jouissant de propriétés analogues. Mais il n'est pas vrai alors, en général, que la trace de $f_{p}$ n'est non nulle que pour les représentations obtenues par torsion de $\pi_{p}$ (prendre $G=G L_{2}, \pi_{p}=$ représentation de Steinberg). Si les représentations $\pi_{p} \otimes \chi_{p}$ sont les seules représentations unitaires ayant cette propriété, la démonstration reste valide. 
2. LE CAS DE GL $(2 n)$ :

\section{REPRÉSENTATIONS ET FONCTIONS À LA PLACE ARCHIMÉDIENNE}

Comme on l'a dit dans l'introduction le reste de l'article est consacré au groupe $\operatorname{GL}(2 n) \operatorname{sur} \mathbb{Q}$. Dans ce chapitre nous voulons imiter dans ce cas les résultats réels du $₫ 1.5$. Puisque $\mathrm{GL}(2 n, \mathbb{R})$, pour $n>1$, n'a pas de série discrète, les méthodes du Chapitre 1 ne s'appliquent pas. On doit donc considérer l'automorphisme extérieur (essentiellement : $g \mapsto{ }^{t} g^{-1}$ ) de GL(2n); tous les objets (représentations, intégrales orbitales, formule des traces, ...) seront les objets tordus pour cet automorphisme.

2.1. Représentations $\theta$-stables. Soit $J$ la matrice antidiagonale

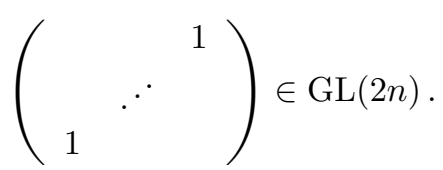

On note $\mathrm{G}$ le groupe $\mathrm{GL}(2 n)$; on le munit de l'automorphisme $g \mapsto \theta(g)=$ $J^{t} g^{-1} J$. Noter que $\theta$ préserve le sous-groupe de Borel usuel de $\mathrm{G}$; il ne préserve pas un épinglage (cf. [KS]) (on revient là-dessus au Chapitre 4).

Nous suivons Waldspurger [W1, ce qui nous permettra d'utiliser ses résultats sans changement.

Soit $\pi$ une représentation (admissible) irréductible de $\mathrm{G}(\mathbb{R})$. Alors $\pi$ est $\theta$ invariante $\left(\pi \cong \pi^{\theta}\right.$ où $\left.\pi^{\theta}=\pi \circ \theta\right)$ si et seulement si $\pi$ est isomorphe à sa duale $\tilde{\pi}$. Notons $\widehat{\mathrm{G}}=\mathrm{GL}(2 n, \mathbb{C})$ le groupe dual. La classification de Langlands associe à $\pi$ une représentation semisimple

$$
r(\pi): \mathrm{W}_{\mathbb{R}} \longrightarrow \widehat{\mathrm{G}} .
$$

D'après des faits bien connus, $\pi \cong \widetilde{\pi}$ si, et seulement si, $r(\pi)$ est isomorphe à $\widetilde{r(\pi)}$.

Soit $p_{1}, \ldots, p_{n} \in \frac{1}{2}+\mathbb{Z}$. Si $p \in \frac{1}{2} \mathbb{Z}$ on lui associe un caractère $\chi$ de $\mathbb{C}^{\times}$, noté d'après Langlands $z \mapsto z^{p}(\bar{z})^{-p}$. C'est un caractère unitaire. Soit $r(\chi)$ la représentation de $\mathrm{W}_{\mathbb{R}}$ induite de $\chi$ : Langlands lui associe une représentation de la série discrète (unitaire) de $\operatorname{GL}(2, \mathbb{R})$, que l'on notera $\delta(p)$. Si $p \in \frac{1}{2}+\mathbb{Z},\left.\chi\right|_{\mathbb{R}^{\times}}$est égal au signe. La formule bien connue

$$
\operatorname{det} \operatorname{ind}(\chi)=\left(\left.\chi\right|_{\mathbb{R} \times}\right) \varepsilon_{\mathbb{C} / \mathbb{R}}
$$

montre que le caractère central de $\delta(p)$ est trivial.

Si $r: \mathrm{W}_{\mathbb{R}} \longrightarrow \mathrm{GL}\left(2 n, \mathbb{C}\right.$ ) est la somme des $r\left(\chi_{i}\right)$ pour $\chi_{i}$ associé à $p_{i}$ (dans $\left.\frac{1}{2}+\mathbb{Z}\right)$, la représentation $\pi$ associée est l'induite unitaire

$$
\operatorname{ind}_{P}^{\mathrm{G}}\left(\delta_{1} \otimes \cdots \otimes \delta_{n}\right) \quad\left(\delta_{i}=\delta\left(p_{i}\right)\right)
$$

où $P$ est le parabolique de type $(2, \ldots, 2)$. Elle est tempérée. Puisque le caractère central de $\delta(p)$ est trivial, celle-ci est autoduale; il en est de même de $\pi$.

La représentation $\pi$ est algébrique au sens de [Clo2, [HT. Supposons de plus les $p_{i}$ distincts. Alors $\pi$ est cohomologique. Plus précisément, soit

$$
p(\pi)=\left(p_{1}, p_{2}, \ldots, p_{n},-p_{n},-p_{2}, \ldots,-p_{1}\right)
$$


où l'on a supposé $p_{1}>p_{2}>\cdots>p_{n}>0$, et

$$
\begin{aligned}
m(\pi) & =\left(p_{1}-\frac{2 n-1}{2}, p_{2}-\frac{2 n-3}{2}, \ldots, p_{n},-\frac{1}{2},-p_{n}+\frac{1}{2}, \ldots,-p_{1}+\frac{2 n-1}{2}\right) \\
& =\left(m_{1}, m_{2}, \ldots, m_{2 n}\right) .
\end{aligned}
$$

Alors $m(\pi)$ est le plus haut poids d'une représentation rationnelle $V$ de GL(2n), et

$$
H^{\bullet}\left(\mathfrak{g}, K_{\infty} ; \pi \otimes V\right) \neq 0
$$

(cf. [Clo2, Lemme 3.14]; noter que $V$ est autoduale). On remarquera que les calculs précédents montrent que toute représentation cohomologique et tempérée de $\mathrm{GL}(2 n)(\mathbb{R})$ est autoduale.

Pour expliquer les calculs qui suivent, décrivons la factorisation de $r=r(\pi)$ donnée par la fonctorialité d'Arthur. Comme on l'a remarqué, chaque représentation $r(\chi)$ est de déterminant trivial; donc

$$
r(\chi): \mathrm{W}_{\mathbb{R}} \longrightarrow \mathrm{SL}(2, \mathbb{C}) .
$$

Ainsi $r(\chi)$ laisse invariante une forme symplectique sur $\mathbb{C}^{2}$; on en déduit que dans une base convenable,

$$
r: \mathrm{W}_{\mathbb{R}} \longrightarrow \mathrm{Sp}(2 n, \mathbb{C}) \subset \mathrm{GL}(2 n, \mathbb{C}) .
$$

Le groupe complexe $\widehat{H}=\operatorname{Sp}(2 n, \mathbb{C})$ est le groupe dual du groupe spécial orthogonal déployé de type $B_{n}$, que l'on notera $H=\mathrm{SO}^{*}(2 n+1)$ (sur $\mathbb{R}$ ou $\mathbb{Q}$ ). Par conséquent $r$ définit un paramètre de Langlands (réel) pour $H$. On vérifie aussitôt que $r$ ne se factorise par aucun sous-groupe de Levi de $\widehat{H}$; d'après Langlands $r$ définit donc un $L$-paquet de séries discrètes de $H(\mathbb{R})$, ou d'ailleurs de toute forme intérieure de celui-ci ; en particulier $r$ définit une représentation de dimension finie $\pi_{H}$ de

$$
H_{c}(\mathbb{R})=\mathrm{SO}(2 n+1, \mathbb{R}) \text {. }
$$

Décrivons celle-ci. Le tore maximal $T_{H}$ de $H_{c}$ s'identifie naturellement à $\left(T_{a}\right)^{n}$ où $T_{a}$ est le tore réel anisotrope de dimension 1 ; la demi-somme des racines, pour un ordre convenable, est alors

$$
\rho_{H}=\left(\frac{2 n-1}{2}, \frac{2 n-3}{2}, \ldots, \frac{1}{2}\right) \in X^{*}\left(T_{H}\right) \otimes \frac{1}{2} \mathbb{Z}=\left(\frac{1}{2} \mathbb{Z}\right)^{n} .
$$

La représentation $\pi_{H}$ a pour plus haut poids $m_{H}=p_{H}-\rho_{H} \in X^{*}\left(T_{H}\right)$, où

$$
p_{H}=\left(p_{1}, \ldots, p_{n}\right) ;
$$

$p_{H}$ est le paramètre du caractère infinitésimal de $\pi_{H}$.

2.2. Application norme. Dans ce paragraphe nous décrivons rapidement suivant Waldspurger [W1, §III.2] (auquel nous renvoyons le lecteur pour les détails) l'application (ou plutôt la correspondance), généralement appelée norme, entre classes de conjugaison tordue dans $\operatorname{GL}(2 n, \mathbb{R})$ et classes de conjugaison dans $\mathrm{SO}^{*}(2 n+1, \mathbb{R})$.

Notons $\mathrm{G}^{+}$le produit semi-direct de $\{1, \theta\} \simeq \mathbb{Z} / 2 \mathbb{Z}$ par $\mathrm{G}, \theta$ opérant par $x \mapsto$ $J^{t} x^{-1} J$. On a $\mathrm{G}^{+}=\mathrm{G} \amalg \theta \mathrm{G}$. Si $g, h \in \mathrm{G}$ on dit que $g$ et $h$ sont $\theta$-conjugués si $g=x^{-1} h x^{\theta}$ pour un $x \in \mathrm{G}$; il revient au même de dire que $\theta g$, $\theta h$ sont conjugués par $\mathrm{G} \subset \mathrm{G}^{+}$. Ces notions ont un sens pour les points à valeurs dans un corps quelconque.

$\mathrm{G}^{+}$étant réductif (non connexe) il y a une notion naturelle d'élément semisimple dans $\mathrm{G}^{+} ; \widetilde{g}=\theta g(g \in \mathrm{G})$ est semisimple si et seulement si $\widetilde{g}^{2}=\left(g^{\theta}\right) g$ est semisimple. On dira que $g$ est $\theta$-semisimple. Si $g \in \theta \mathrm{G}$ est semisimple, son centralisateur $Z_{\mathrm{G}}(g)$ 
dans $\mathrm{G}$ est réductif. On dit que $g$ est fortement régulier si c'est un tore $11 \mathrm{Si} g, h$ sont fortement réguliers, on dit qu'ils sont stablement conjugués s'il existe $x \in \mathrm{G}(\mathbb{C})$ tel que $x g x^{-1}=h$. On fait des définitions analogues sur $\mathbb{Q}$ et sur un corps $p$-adique; cf. W1. Par transport de structure on a donc défini des éléments "stablement $\theta$-conjugués", " $\theta$-fortement réguliers" dans $\mathrm{G}(\mathbb{R})$.

On sait définir de même des éléments fortement réguliers, ainsi que la conjugaison stable, dans $H(\mathbb{R})$ (Kottwitz $[\mathrm{K} 2]$ ).

Soit $g \in \mathrm{G}(\mathbb{R})$ un élément fortement $\theta$-régulier, et soit $\Lambda(g)$ l'ensemble des valeurs propres (complexes) de $g^{\theta} \cdot g$ : celles-ci sont distinctes. Alors il existe $h \in H(\mathbb{R})$ tel que l'ensemble des valeurs propres $\Lambda(h)$ de $h$ soit égal à $-\Lambda(g) \cup\{1\}$. On dit que $h$ est une norme de $g$.

Notation 2.3. $h=\mathcal{N} g$.

Proposition 2.4 (Waldspurger). La correspondance $\mathcal{N}$ définit une bijection entre classes de $\theta$-conjugaison stable d'éléments fortement $\theta$-réguliers (dans $\mathrm{G}$ ) et classes de conjugaison stable d'éléments fortement réguliers (dans $H$ ).

On dit qu'un élément (fortement $\theta$-régulier) de $\mathrm{G}$ est elliptique si sa norme est une classe elliptique de $H(\mathbb{R})$. On peut alors la considérer comme une classe de conjugaison dans $H_{c}(\mathbb{R})$, conjugaison et conjugaison stable étant identiques dans un groupe compact.

2.5. Stabilité des caractères tordus $\Theta_{\pi, \theta}$. Soit $\pi$ une représentation algébrique, régulière, tempérée et (donc) autoduale $(\$ 2.1)$ de $\mathrm{G}(\mathbb{R})$. Le choix d'un opérateur d'entrelacement involutif $A: \mathfrak{H}_{\pi} \longrightarrow \mathfrak{H}_{\pi}\left(A^{2}=1\right)$ entrelaçant $\pi$ en $\pi \circ \theta$ permet d'étendre $\pi$ en une représentaton $\pi^{+}$de $\mathrm{G}^{+}(\mathbb{R})$. On note $\Theta_{\pi, \theta}$ le caractère de $\pi^{+}$ $\operatorname{sur} \theta \mathrm{G}(\mathbb{R})$ :

$$
\Theta_{\pi, \theta}(g)=\Theta_{\pi^{+}}(\theta g) \quad(g \in \mathrm{G}(\mathbb{R})) .
$$

A priori, $\Theta_{\pi, \theta}$ est une distribution en $g$, mais un théorème d'Harish-Chandra (cf. Bouaziz [Bou] ) montre que c'est une fonction analytique sur les éléments (fortement) $\theta$-réguliers.

Théorème 2.6. Pour un choix convenable de $A$, on a pour tout $g \in \mathrm{G}(\mathbb{R})$ de norme fortement régulière et elliptique :

$$
\Theta_{\pi, \theta}(g)=\Theta_{\pi_{H}}(\mathcal{N} g) .
$$

En particulier, $\Theta_{\pi, \theta}$ est invariant par conjugaison (tordue) stable sur les éléments de norme elliptique.

Le théorème résulte du travail de Bouaziz [Bou, qu'il nous suffit d'interpréter. Comme l'article de Bouaziz est écrit dans un langage très différent de la théorie usuelle des représentations admissibles (construction des représentations par la méthode de Duflo-Kirillov) nous serons succincts, renvoyant le lecteur à [Bou pour les détails.

Donnons un ensemble explicite de représentants pour les classes de conjugaison tordue d'éléments fortement réguliers. Dans $T_{H_{c}} \cong\left(T_{a}(\mathbb{R})\right)^{n}$, dont on représente les éléments par $\left(w_{1}, \ldots, w_{n}\right): w_{i} \in \mathbb{C},\left|w_{i}\right|=1$, un élément est fortement régulier si $w_{i} \neq w_{j}, w_{i}^{2} \neq 1$. Dans $\operatorname{GL}(2, \mathbb{R})$, soit $J_{2}$ la matrice $\left(\begin{array}{ll}0 & 1 \\ 1 & 0\end{array}\right)$ et considérons le

\footnotetext{
${ }^{11}$ Cette définition diffère en apparence de celle de Waldspurger [W1 §I.1], mais sa classification des centralisateurs $Z_{\mathrm{G}}(g)$ ([W1 fin du §I.3]) montre qu'elle est équivalente pour $\mathrm{GL}(2 n)$.
} 
sous-groupe $\mathrm{O}(2, \mathbb{R})=J_{2} \mathrm{SO}(2, \mathbb{R}) \cup \mathrm{SO}(2, \mathbb{R})$. Si $\theta$ est notre automorphisme usuel pour GL(2), on a alors :

$$
\begin{gathered}
g \in \mathrm{SO}(2, \mathbb{R}) \Longrightarrow\left(g^{\theta}\right) g=\left(J_{2} g J_{2}\right) g=1, \\
g=J_{2} h, h \in \mathrm{SO}(2, \mathbb{R}) \Longrightarrow g^{\theta} g=h^{2} .
\end{gathered}
$$

On en déduit que la norme est dans ce cas surjective de $J_{2} \mathrm{SO}(2, \mathbb{R})$ vers $T_{H_{c}} \cong$ $T_{a}(\mathbb{R})$; si $R_{\alpha}$ est la rotation d'angle $\alpha$, elle envoie $J_{2} R_{\alpha}$ vers $-e^{2 i \alpha}$.

Dans $\mathrm{GL}(2 n, \mathbb{R})$, on considère le tore $T_{G} \cong T_{a}(\mathbb{R})^{n}$ donné par les rotations d'angle $\left(\alpha_{1}, \ldots \alpha_{n}\right)$ dans la base $\mathcal{B}=\left(e_{1}, e_{2 n} ; e_{2}, e_{2 n-1} ; \ldots ; e_{n}, e_{n+1}\right)$. Si $R_{\alpha}(\alpha=$ $\left.\left(\alpha_{1}, \ldots, \alpha_{n}\right)\right)$ est une telle rotation, on a alors

$$
\mathcal{N}\left(J R_{\alpha}\right)=\left(-e^{2 i \alpha_{1}},-e^{2 i \alpha_{2}}, \ldots,-e^{2 i \alpha_{n}}\right)
$$

vu comme élément de $T_{H_{c}}$.

À la représentation $\pi$, la méthode des orbites associe une forme linéaire $f \in \mathfrak{g}^{*}$ où $\mathfrak{g}=\operatorname{Lie}(\mathrm{G} / \mathbb{R})=\mathrm{M}_{2 n}(\mathbb{R})$. Elle est définie ainsi. Soit $\mathfrak{h}$ la sous-algèbre de Cartan de $\mathfrak{g}$ isomorphe à $\mathbb{C}^{n}$, obtenue à l'aide d'un plongement d'algèbres $\mathbb{C} \subset M_{2}(\mathbb{R})$ et de la base $\mathcal{B}$ ci-dessus. Alors $f: \mathfrak{h} \longrightarrow \mathbb{R}$ est définie par

$$
\left(z_{1}, \ldots, z_{n}\right) \mapsto \sum_{i} p_{i}\left(z_{i}-\bar{z}_{i}\right) / \sqrt{-1}=\sum_{i} 2 p_{i} \operatorname{Im}\left(z_{i}\right) \quad\left(z_{i} \in \mathbb{C}\right) ;
$$

c'est donc (au facteur $\sqrt{-1}$ près) la différentielle de la représentation induisant $\pi$. On étend $f$, de façon naturelle, en une forme linéaire sur $\mathfrak{g}$ par dualité de Killing. Le stabilisateur $\mathrm{G}^{+}(f)$ est alors $T_{\mathbb{C}} \amalg \theta J T_{\mathbb{C}}, T_{\mathbb{C}}=\left(\mathbb{C}^{\times}\right)^{n}$ étant le tore exponentielle de $\mathfrak{h}$.

La forme bilinéaire alternée $B_{f}(X, Y)=f([X, Y])$ sur $\mathfrak{g}$ est non-dégénérée sur $\mathfrak{g} / \mathfrak{g}(f)$, où $\mathfrak{g}(f)=\operatorname{Lie} G^{+}(f)$. Soit $M$ le revêtement métaplectique de $\operatorname{Sp}(\mathfrak{g} / \mathfrak{g}(f))$. On obtient alors une extension centrale

$$
1 \longrightarrow\{1, \varepsilon\} \longrightarrow \widetilde{\mathrm{G}}(f) \longrightarrow \mathrm{G}^{+}(f) \longrightarrow 1
$$

([Bou, p. 47]; Bouaziz note différemment $\widetilde{\mathrm{G}}(f)$ ). L'application de chacun des facteurs $\mathbb{C}_{u}^{\times}=\{w:|w|=1\} \subset \mathbb{C}^{\times}$de $T_{\mathbb{C}}$ dans $\operatorname{Sp}(\mathfrak{g} / \mathfrak{g}(f))$ est quadratique (i.e., passe au quotient par $\left.\left(\mathbb{C}_{u}^{\times}\right) / \pm 1\right)$. On en déduit que ce revêtement est scindé au-dessus de $T_{\mathbb{C}}$, puis au-dessus de $T_{\mathbb{C}} \amalg \theta \cdot J \cdot T_{\mathbb{C}}$. Il existe donc un caractère unitaire $\tau$ de $\widetilde{\mathrm{G}}(f)$ tel que

$$
\begin{aligned}
& \tau(\varepsilon)=-1, \\
& \tau(z)=\chi(z) \quad\left(z \in T_{\mathbb{C}}\right)
\end{aligned}
$$

où $\chi$ est le produit des $\chi_{i}=z^{p_{i}}(\bar{z})^{-p_{i}}$.

Soit $s=s\left(\alpha_{1}, \ldots, \alpha_{n}\right) \in \theta \mathrm{G}, s=\theta \cdot J R_{\alpha}, R_{\alpha}$ étant la matrice de rotation cidessus. Soit $W$ le groupe de Weyl de $\left(\mathrm{G}, T_{\mathbb{C}}\right)$ : il s'identifie à $\mathfrak{S}_{n} \times\{ \pm 1\}^{n}:=W_{H}$. Par ailleurs la donnée de $\tau$ définit une extension $\pi^{+}$de $\pi$ à $\mathrm{G}^{+}$. Sa construction, due à Duflo, est difficile. Elle est rappelée par Bouaziz dans [Bou, §5.4]. Alors, d'après Bou, Prop. 6.1.2],

$$
\Theta_{\pi^{+}}\left(\theta J R_{\alpha}\right)=\Theta_{\pi, \theta}\left(J R_{\alpha}\right)=\sum_{w \in W_{H}} \frac{\varepsilon(w)^{w} \tau(s) \rho_{\mathrm{l}}(s)}{D(s)} .
$$

La valeur de $\rho_{\mathfrak{l}}(s)$ et de $D(s)$ dépend du choix d'un sous-espace lagrangien $\mathfrak{l} \subset$ $\mathfrak{g} \otimes \mathbb{C}\left(\right.$ pour $\left.B_{f}\right)$ stable par $s ;$ soit $\mathfrak{g}=\mathfrak{g}^{s} \oplus \mathfrak{q}$ la décomposition de $\mathfrak{g}$ selon les espaces 
propres de $s$. Alors

$$
D(s)=\left.\operatorname{det}(1-s)\right|_{\mathfrak{r} \mathfrak{q}} .
$$

Il est clair que $D(s)$ est invariant par conjugaison stable (pour des choix compatibles de $\mathfrak{l})$. Par transport de structure on le calcule sur un élément de $\mathrm{GL}(2 n, \mathbb{C})$ de la forme $\theta x$ où

$$
x=\left(\begin{array}{cccccc}
x_{1} & & & & & \\
& \ddots & & & & \\
& & x_{n} & & & \\
& & & x_{n}^{-1} & & \\
& & & & \ddots & \\
& & & & & x_{1}^{-1}
\end{array}\right) .
$$

On prend $\mathfrak{l}$ égale au radical unipotent de l'algèbre de Borel standard de $\mathrm{M}_{2 n}(\mathbb{C})$ : on calcule les valeurs propres sur $\mathfrak{l}$ de l'endomorphisme

$$
X \longmapsto-J \operatorname{Ad}(x)^{t} X J .
$$

Un calcul simple donne alors

$$
D(x)=\prod_{i=1}^{n}\left(1+x_{i}^{2}\right) \prod_{i<j}\left(1-x_{i}^{2} / x_{j}^{2}\right) \prod_{i<j}\left(1-x_{i}^{2} x_{j}^{2}\right)
$$

qui n'est autre que le dénominateur de Weyl $\prod\left(1-e^{\alpha}\right)$ (le produit portant sur les racines positives de $H)$ évalué en $\left(-x_{i}^{2}\right)=\mathcal{N} x$. Le terme $\rho_{\mathfrak{l}}(s)^{-1}$ le transforme en $\prod\left(e^{-\alpha / 2}-e^{\alpha / 2}\right):=D_{H}$. Par ailleurs,

$$
\tau(s)=\tau(\theta J) \tau\left(R_{\alpha}\right)=\tau(\theta J) \prod e^{2 \sqrt{-1} \alpha_{i} p_{i}}=\varepsilon \chi_{H}\left(\mathcal{N}\left(J R_{\alpha}\right)\right)
$$

où $\chi_{H}$ est le caractère de $T_{H}$ paramétré par $\left(p_{1}, \ldots, p_{n}\right)$ et $\mathcal{N}\left(J R_{\alpha}\right)=\left(-e^{2 \sqrt{-1} \alpha_{i}}\right)$. (Noter que $p$ paramètre le caractère infinitésimal de $\pi_{H}$; donc $p=m+\rho_{H}$ où $m$ est le plus haut poids. L'indétermination due au fait que $p$ n'est pas un caractère disparait puisqu'on évalue en des carrés.) Enfin, on vérifie que $\varepsilon(w)$ coïncide, au signe près, avec le déterminant sur $W_{H}$ (voir [Bou, (5.5.1) et p. 46]). L'expression (2.1) donne alors

$$
\Theta_{\pi, \theta}(x)=\varepsilon \sum_{w} \frac{\varepsilon(w) \mathcal{N}(x)^{w \chi_{H}}}{D(\mathcal{N}(x))}, \quad \varepsilon= \pm 1
$$

d'où, d'après la formule de Weyl, le Théorème.

2.7. Pseudo-coefficients et intégrales orbitales tordues. La représentation $\pi$ de $G(\mathbb{R})$ reste fixée (pour simplifier on note simplement $G$ le groupe $G(\mathbb{R})$ ). On vérifie aisément que $\pi$ est $\theta$-discrète, i.e., isolée parmi les représentations tempérées, $\theta$-invariantes de G. D'après le théorème de Paley-Wiener de Mezo [M], il existe une fonction $f_{\pi} \in \mathcal{C}_{c}^{\infty}(\mathrm{G}), K_{\infty}$-finie (pour un sous-groupe compact maximal $K_{\infty}$ de $\mathrm{G}(\mathbb{R})$ ) telle que

$$
\operatorname{trace}\left(\pi\left(f_{\pi}\right) A\right)=1
$$

et

$$
\operatorname{trace}\left(\rho\left(f_{\pi}\right) A_{\rho}\right)=0
$$


pour toute représentation tempérée, irréductible, $\theta$-invariante $\rho \neq \pi$ de $\mathrm{G}$; on note $A$ l'opérateur d'entrelacement entre $\pi$ et $\pi \circ \theta$, normalisé par le Théorème 2.6. et $A_{\rho}$ un opérateur d'entrelacement (non nul) entre $\rho$ et $\rho \circ \theta$.

Soit $\gamma \in \mathrm{G}$ un élément $\theta$-semisimple. Son centralisateur tordu $I$, égal par définition à la composante neutre de $I^{\prime}=\left\{g \in \mathrm{G}: g^{-1} \gamma g^{\theta}=\gamma\right\}$ est alors réductif. On considère l'intégrale orbitale tordue (pour des mesures de Haar $d g$ et $d i$ arbitraires)

$$
\mathrm{TO}_{\gamma}(f)=\int_{I \backslash \mathrm{G}} f\left(g^{-1} \gamma g^{\theta}\right) \frac{d g}{d i}
$$

pour $f \in C_{c}^{\infty}(\mathrm{G})$. Si $\gamma$ est fortement régulier, $I=I^{\prime}$ est un tore.

Soit $P=M N \subset \mathrm{G}$ un parabolique $\theta$-stable et soit $\gamma \in M$ un élément fortement $\theta$-régulier. Alors $\gamma$ a la même propriété relativement à $M$, et son centralisateur tordu est un tore de $M$. Si $f \in C_{c}^{\infty}(\mathrm{G})$, soit $\bar{f}(x)=\int_{K_{\infty}} f\left(k^{-1} x k^{\theta}\right) d k$ (pour la mesure de Haar normalisée). Alors

$$
\mathrm{TO}_{\gamma}(f)=\int_{I \backslash M N} \bar{f}\left(n^{-1} m^{-1} \gamma m^{\theta} n^{\theta}\right) \frac{d m d n}{d i} .
$$

Si $h \in \mathcal{C}_{c}^{\infty}(P)$ et $m \in M$,

$$
\int_{N} h\left(n^{-1} m n^{\theta} m^{-1}\right) d n=D(m)^{-1} \int_{N} h(n) d n
$$

où $D(m)=|\operatorname{det}(1-\operatorname{Ad}(m) \circ \theta)|_{\mathfrak{n}}$ et $\mathfrak{n}=\operatorname{Lie}(N) ; D(m)$ est non nul si $m$ est $\theta$-régulier. Ainsi

$$
\operatorname{TO}_{\gamma}(f)=D(\gamma)^{-1} \int_{I \backslash M} \bar{f}^{(P)}\left(m^{-1} \gamma m^{\theta}\right) \frac{d m}{d i}=D(\gamma)^{-1} \operatorname{TO}_{\gamma}^{M}\left(\bar{f}^{(P)}\right)
$$

où l'intégrale orbitale tordue est prise dans $M$ et $f^{(P)}$ est définie selon HarishChandra par

$$
f^{(P)}(m)=\int_{N} f(n m) d n .
$$

Lemme 2.8. Soit $\gamma$ un élément fortement $\theta$-régulier non-elliptique de G. Alors $\gamma$ est $\theta$-conjugué à un élément (fortement $\theta$-régulier) de la composante de Levi $M$ d'un parabolique $\theta$-stable propre de $\mathrm{G}$.

Preuve. Soit en effet $\delta=\gamma^{\theta} \gamma$. C'est un élément régulier de GL(2n, R $)$ dont l'ensemble des valeurs propres (distinctes et complexes) est autodual et dont au moins une valeur propre $\lambda \in \mathbb{C}^{*}$ n'est pas de module 1 . Posons $i=1$ ou 2 selon que $\lambda$ est réel ou non et considérons le parabolique supérieur standard de type $(i, 2 n-2 i, i)$ de $\mathrm{G}$; il est $\theta$-stable. À conjugaison près sur $\delta$ (et donc à $\theta$-conjugaison près sur $\gamma$ ), on peut supposer que $\delta$ est un élément du sous-groupe de Levi standard $M$ de ce parabolique :

$$
\delta=\left(\begin{array}{ccc}
\lambda & & \\
& * & \\
& & \lambda^{-1}
\end{array}\right)
$$

(si $i=2$ on choisit un plongement de $\mathbb{C}$ dans $\mathrm{M}_{2}(\mathbb{R})$ ). Comme $\delta^{\theta} \gamma=\gamma \delta$ et $\delta$ est régulier, cela impose que $\gamma \in M$. 
Lemme 2.9. Si $\gamma$ est fortement $\theta$-régulier et non-elliptique,

$$
\mathrm{TO}_{\gamma}\left(f_{\pi}\right)=0 .
$$

Preuve. D'après (2.4) cette intégrale orbitale se calcule dans $M$, où $P=M N$ est un parabolique propre, $\theta$-stable et $\gamma \in M$. D'après $\mathrm{KR}$ (et grâce au théorème de Paley-Wiener de Mezo déjà cité) une fonction $h$ sur $M$ a des intégrales orbitales tordues nulles si trace $\left(\pi_{M}(h) A\right)=0$ pour toute représentation $\theta$-stable tempérée $\pi_{M}$ de $M, A \neq 0$ étant un opérateur entrelaçant $\pi_{M}$ et $\pi_{M} \circ \theta$. Pour $h=\bar{f}^{(P)}$, un lemme bien connu d'Harish-Chandra (qui s'étend formellement au cas tordu) donne

$$
\operatorname{trace}\left(\pi_{M}(h) A\right)=\operatorname{trace}\left(\pi_{G}(f) A_{G}\right)
$$

où $\pi_{G}$ est induite de $\pi_{M}$ et $A_{G}$ est l'opérateur d'entrelacement induit. Mais l'expression de droite s'annule d'après (2.3).

Soit $\gamma \in \mathrm{G}$ un élément $\theta$-semisimple. On dit que $\gamma$ est $\theta$-elliptique si la composante déployée du centre de son centralisateur tordu est réduite à l'élément neutre. L'élément $\delta=\gamma^{\theta} \gamma$ de $\operatorname{GL}(2 n, \mathbb{R})$ est conjugué à un élément diagonal de $\operatorname{GL}(2 n, \mathbb{C})$ de la forme $\left(x_{1}, \ldots, x_{n}, x_{n}^{-1}, \ldots, x_{1}^{-1}\right)$. Il définit donc une classe de conjugaison $\mathcal{N} \gamma$ dans $\mathrm{SO}(2 n+1, \mathbb{C})$, par son spectre

$$
\Lambda(\mathcal{N} \gamma)=\left\{-x_{1}, \ldots,-x_{1}^{-1}\right\} \cup\{1\} .
$$

On vérifie que $\mathcal{N} \gamma$ (qui est toujours conjugué à un élément de $\mathrm{SO}^{*}(2 n+1, \mathbb{R})$ ) est conjugué à un élément, unique à conjugaison près de $\mathrm{SO}(2 n+1, \mathbb{R})$ si, et seulement si, $\gamma$ est $\theta$-elliptique 12

Lemme 2.10. Si $\gamma \in \mathrm{G}$ est $\theta$-semisimple mais non $\theta$-elliptique, $\mathrm{TO}_{\gamma}\left(f_{\pi}\right)=0$.

Preuve. Soit en effet $I$ le centralisateur tordu de $\gamma$. Une fonction $f \in C_{c}^{\infty}(\mathrm{G})$ étant donnée, on peut trouver une fonction $h \in \mathcal{C}_{c}^{\infty}(I)$ telle que, pour $x \in I$, voisin de 1 ,

$$
\mathrm{TO}_{x \gamma}(f)=\mathrm{O}_{x}^{I}(h)
$$

le membre de droite étant une intégrale orbitale ordinaire dans $I$ (voir la preuve de la Proposition 3.11 pour l'argument). Si $x \gamma$ est fortement régulier, $x$ est régulier dans $I$; pour $x$ assez proche de 1 , les composantes neutres du centralisateur de $x$ et du centralisateur tordu de $x \gamma$ coïncident ([La1, Cor. 3.1.5]). Puisque la composante déployée du centre de $I$ est non-triviale, les intégrales orbitales $\mathrm{O}_{x}^{I}(h)$ sont donc nulles (au voisinage de 1 ) en les éléments réguliers et donc $h(1)=\mathrm{TO}_{\gamma}(f)$ (pour des normalisations convenables des mesures) s'annule.

Si $\gamma \in \mathrm{G}$ est $\theta$-semisimple, l'intégrale orbitale tordue stable de $f$ associée à $\gamma$ est définie par Labesse [La1, §2.7]. On la note

$$
\operatorname{STO}_{\gamma}(f) \text {. }
$$

\footnotetext{
${ }^{12}$ Posons $\delta=\gamma^{\theta} \gamma$. La conjugaison par $\theta \gamma$ induit une involution sur $\mathrm{G}_{\delta}$ (un sous-groupe de Levi de $\mathrm{GL}_{2 n}$ ) dont le sous-groupe des points fixes est le centralisateur tordu $\mathrm{G}_{\gamma}$ de $\gamma$. La structure des anti-involutions des algèbres semisimples complexes montre que le centre $Z$ de $\mathrm{G}_{\gamma}$ coïncide avec le sous-groupe des $\theta \gamma$-invariants du centre $Z^{\prime}$ de $\mathrm{G}_{\delta}$. Or $Z^{\prime}=\mathbb{G}_{m} D$ où $D$ désigne l'adhérence Zariski du sous-groupe engendré par $\delta$ (central, fixé par $\theta \gamma$ ). Ainsi $Z=Z^{\prime \gamma \theta}=\mathbb{G}_{m}^{\theta \gamma} D=\{ \pm 1\} D$, ce qui conclut.
} 
2.11. Intégrales orbitales tordues en les éléments elliptiques. Nous pouvons maintenant démontrer le résultat principal de ce chapitre.

Théorème 2.12. Soit $\gamma$ un élément $\theta$-semisimple de $\mathrm{G}(\mathbb{R})$.

(i) Si $\gamma$ n'est pas $\theta$-elliptique, $\mathrm{TO}_{\gamma}\left(f_{\pi}\right)=\operatorname{STO}_{\gamma}\left(f_{\pi}\right)=0$.

(ii) Soit $\gamma$ un élément $\theta$-elliptique, et $I=I_{\gamma}$. Pour un choix convenable de mesures positives sur $\mathrm{G}$ et $I_{\gamma}$,

$$
\mathrm{TO}_{\gamma}\left(f_{\pi}\right)=e(\gamma) \Theta_{\pi_{H}}(\mathcal{N} \gamma)
$$

$\pi_{H}$ étant la représentation (de dimension finie) de $\mathrm{SO}(2 n+1, \mathbb{R})$ associée à $\pi$, et $e(\gamma)= \pm 1$ un signe indépendant de $\pi$. En particulier, ces intégrales orbitales sont stables.

Nous ne décrirons pas la normalisation des mesures, qui résulte de [La1, §A.1]. L'important est que, $\gamma$ étant fixé, celle-ci ne dépend pas de $\pi$.

La partie (i) a déjà été démontrée. Considérons d'abord le cas où $\pi_{0}$ est l'unique représentation tempérée de $\mathrm{G}(\mathbb{R})$ ayant de la cohomologie à coefficients triviaux : c'est celle définie par $p\left(\pi_{0}\right)=\left(\frac{2 n-1}{2}, \frac{2 n-3}{2}, \ldots, \frac{1-2 n}{2}\right)$ (\$2.1). Dans ce cas Labesse [La1] a donné une construction de $f_{\pi_{0}}$ par voie cohomologique, qui permet d'en calculer les intégrales orbitales tordues La1, Thm. A.1.1, ce qui démontre le théorème dans ce cas, $\pi_{0, H}$ étant la représentation triviale 13 Nul doute que l'on pourrait l'étendre au cas général : comme nous avons démontré des résultats plus puissants, nous donnons une démonstration différente.

Pour $\pi$ donnée, soit $(\rho, V)$ la représentation algébrique $(\theta$-stable) telle que la cohomologie de $\pi$ à coefficients dans $V$ soit non nulle; on définit de même $\left(\rho_{H}, V_{H}\right)$, $\rho_{H}$ s'identifiant dans ce cas à $\pi_{H}$. On peut réaliser $V$, à l'aide du théorème de BorelWeil, dans la cohomologie de $\mathrm{G}(\mathbb{C}) / B(\mathbb{C})$ à coefficients dans le fibré en droites $\mathcal{L}_{m}$ où $m=m(\pi)$. Noter que $m$ est invariant par $\theta$; on en déduit naturellement une représentation de $\mathrm{G}^{+}(\mathbb{C})$. Pour $g \in \mathrm{G}(\mathbb{C}), \theta$-régulier, la trace $(g \times \theta \mid V)$ se calcule à l'aide du théorème d'Atiyah-Bott; un calcul simple montre que les points fixes sont paramétrés par le centralisateur de $\theta$ dans $W(\mathrm{G}(\mathbb{C})) \cong \mathfrak{S}_{2 n}$, isomorphe à $W_{H}$. On obtient alors

$$
\text { trace }(g \times \theta \mid V)=\operatorname{trace}\left(\mathcal{N} g \mid V_{H}\right) \text {. }
$$

Soit alors $\Theta_{\rho, \theta}$ le caractère tordu de $\rho$ (pour ce choix d'opérateur d'entrelacement), et soit

$$
g_{\pi}=\Theta_{\rho, \theta} f_{0},
$$

où $f_{0}=f_{\pi_{0}}$. Alors $g_{\pi}$ a les propriétés du Théorème 2.12 relativement à $\pi$. Pour montrer que $g_{\pi}$ a les mêmes intégrales orbitales que $f_{\pi}$, il suffit de montrer que pour toute représentation tempérée $\theta$-stable $\tau$ (et opérateur d'entrelacement $A_{\theta}$ associé) :

$$
\operatorname{trace}\left(\tau\left(f_{\pi}\right) A_{\theta}\right)=\operatorname{trace}\left(\tau\left(g_{\pi}\right) A_{\theta}\right)
$$

(le fait que ceci implique l'égalité des intégrales orbitales tordues est le théorème de densité de Kottwitz-Rogawski [KR]).

On dit que $\tau$ est $\theta$-discrète si elle n'est pas induite d'une représentation $\theta$-stable d'un parabolique $\theta$-stable propre. Dans ce cas le caractère tordu est à support

\footnotetext{
${ }^{13}$ Dans cet article notre $I$ est remplacé par le « $\theta$-centralisateur stable $»$; cf. [La1 p. 52]. La description des centralisateurs tordus par Waldspurger [W1 §I] montre qu'ils coïncident pour $\mathrm{GL}(2 n)$ avec nos centralisateurs connexes.
} 
dans les éléments non $\theta$-elliptiques; donc (2.6) est évidente puisque les intégrales orbitales tordues correspondantes sont nulles. Les représentations $\theta$-discrètes sont, comme on le voit aisément, de la forme

$$
\tau=\operatorname{Ind}\left(\delta_{1}, \ldots, \delta_{n}\right),
$$

où $\delta_{i}$ est une représentation de $\mathrm{GL}(2, \mathbb{R})$ de la série discrète associée à la représentation de $\mathrm{W}_{\mathbb{R}}$ induite d'un caractère $z \mapsto z^{p_{i}}(\bar{z})^{-p_{i}}$ de $\mathrm{W}_{\mathbb{C}}=\mathbb{C}^{*}$, avec $p_{i} \in \frac{1}{2} \mathbb{Z}$, les $p_{i}$ étant distincts, ou bien de la forme

$$
\tau=\operatorname{Ind}\left(\delta_{1}, \ldots, \delta_{n-1}, 1, \varepsilon\right),
$$

les $\delta_{i}$ étant comme auparavant, et $\varepsilon$ étant le caractère d'ordre 2 de $\mathbb{R}^{*}$. Si les $p_{i}$ appartiennent à $\frac{1}{2}+\mathbb{Z}$ (et donc $\tau$ est cohomologique), on a d'une part

$$
\operatorname{trace}\left(\tau\left(f_{\pi}\right) A_{\theta}^{\tau}\right)=\delta(\tau, \pi)
$$

où $\delta$ est le symbole de Kronecker et $f_{\pi}$ est normalisée par $A_{\theta}^{\pi}$; par ailleurs,

$$
\operatorname{trace}\left(\tau\left(g_{\pi}\right) A_{\theta}^{\tau}\right)=\int_{\mathrm{G}} \Theta_{\tau, \theta}(g) \Theta_{\rho, \theta}(g) f_{0}(g) d g .
$$

Mais les intégrales orbitales tordues de $f_{0}$ s'annulent pour $g$ non $\theta$-elliptique; si $g$ est $\theta$-elliptique régulier (donc de centralisateur tordu $\mathrm{U}(1)^{n}=T$ ) et si la mesure sur $T$ est normalisée, on trouve ([La1, Thm. A.1.1])

$$
\mathrm{TO}_{g}\left(f_{0}\right)=1
$$

( $f_{0}$ étant bien sûr le pseudo-coefficient associé à une mesure $d g$ qui est celle définissant l'intégrale obitale). Donc (2.9) s'écrit

$$
\int_{\mathrm{G}} \Theta_{\tau, \theta}(g) \Theta_{\rho, \theta}(g) f_{0}(g) d g=\frac{1}{|W|} \int_{T}\left\{\sum_{\mathcal{N} \delta=\gamma} \Theta_{\tau, \theta}(\delta) \Theta_{\rho, \theta}(\delta) \Delta(\gamma)\right\} d \gamma,
$$

où $\Delta(\gamma)$ est un dénominateur de Weyl (pour la formule d'intégration de Weyl relative à la conjugaison tordue), qu'on vérifie être égal à un facteur $2^{n}$ près (le nombre de $\delta$ de norme $\gamma$ ) au dénominateur de Weyl pour $\mathrm{SO}(2 n+1)$. Le Théorème 2.6 et la relation (2.5) impliquent alors que trace $\left.\left(\tau\left(g_{\pi}\right) A_{\theta}^{\tau}\right)\right)=\delta(\tau, \pi)$, d'après les relations d'orthogonalité sur $\mathrm{SO}(2 n+1)$. Considérons enfin les autres représentations $\tau$ de type (2.7) ou (2.8). On a

$$
\begin{aligned}
\operatorname{trace}\left(\tau\left(g_{\pi}\right) A_{\theta}^{\tau}\right) & =\operatorname{trace}\left(\int_{\mathrm{G}} \tau(x) g_{\pi}(x) A_{\theta}^{\tau} d x\right) \\
& =\operatorname{trace}\left(\int_{\mathrm{G}} \tau(x) f_{0}(x) \operatorname{trace}\left(\rho(x) A_{\theta}^{\rho}\right) A_{\theta}^{\tau} d x\right) \\
& =\operatorname{trace}\left(\int_{\mathrm{G}} f_{0}(x)(\tau(x) \otimes \rho(x)) A_{\theta}^{\tau} \otimes A_{\theta}^{\rho} d x\right) .
\end{aligned}
$$

Si $\tau$ est du type indiqué et non cohomologique, son caractère infinitésimal $\lambda$ (la somme des $p_{i}$ et des $-p_{i}$, avec $p=0$ pour les caractères $\left.1, \varepsilon\right)$ n'appartient pas à $\left\{\frac{1}{2}+\mathbb{Z}\right\}^{2 n}$. On sait que les caractères infinitésimaux des sous-quotients de $\tau \otimes \rho$ sont de la forme $\lambda+\mu$ où $\mu$ est un poids (entier) de $\rho$. Il a donc la même propriété; la trace de $f_{0}$ dans $\tau \otimes \rho$ est donc nulle, ce qui termine la démonstration.

Si $T \subset \mathrm{G}$ est le centralisateur tordu d'un élément $\theta$-semisimple fortement régulier et si $\mathcal{D}(T, \mathrm{G}, \mathbb{R})$ classifie la conjugaison stable (modulo conjugaison) pour les éléments de $T$, on a $\mathcal{D}(T, \mathrm{G}, \mathbb{R})=H^{1}(\mathbb{R}, T)$ puisque $G$ est cohomologiquement trivial. Avec les notations de Labesse [La1, pp. 122-123], $H^{1}(\mathbb{R}, T)=\mathcal{E}(T, G, \mathbb{R}) ;$ si $\kappa$ est un élément du dual de $\mathcal{E}$, on en déduit : 
Corollaire 2.13. Si $\kappa \neq 1$,

$$
\mathrm{TO}_{\delta, \kappa}\left(f_{\pi}\right)=\sum_{d \in \mathcal{D}(T, \mathrm{G}, \mathbb{R})}\langle\kappa, d\rangle \mathrm{TO}_{d \delta}\left(f_{\pi}\right)=0 .
$$

Un argument de descente (ibid.) montre que le même résultat d'annulation s'étend à tous les éléments $\delta$ de norme elliptique. La fonction $f_{\pi}$ est donc stabilisante au sens de Labesse.

\section{Asymptotique de la formule des traces tordues de GL $(2 n)$}

Dans ce chapitre, nous démontrons le Théorème B énoncé dans l'introduction en admettant le Théorème E.

3.1. Énoncé du Théorème. Soient $\ell$ et $p$ deux nombres premiers distincts, $n \geq 1$ un entier. On considère à nouveau le groupe algébrique $G:=\mathrm{GL}_{2 n}$ sur $\mathbb{Q}$ muni de son $\mathbb{Q}$-automorphisme d'ordre 2

$$
\theta(g)=J^{t} g^{-1} J^{-1},
$$

où $J=J^{-1} \in \mathrm{GL}_{2 n}(\mathbb{Q})$ est la matrice antidiagonale définie au 2.1 On définit encore $G^{+}$comme étant le $\mathbb{Q}$-groupe produit semi-direct de $\mathbb{Z} / 2 \mathbb{Z}=\langle\theta\rangle$ par $G$ défini par $\theta$.

À la place $p$, fixons une représentation irréductible supercuspidale $\omega$ de $\mathrm{GL}_{n}\left(\mathbb{Q}_{p}\right)$ dont la contragrédiente $\check{\omega}$ n'est isomorphe à aucune tordue non ramifiée de $\omega$. Soient $P$ le parabolique triangulaire supérieur de $G$ de type $(n, n)$ et $M=\mathrm{GL}_{n} \times \mathrm{GL}_{n}$ son sous-groupe de Levi diagonal. Si $\chi$ est un caractère non ramifié de $\mathrm{GL}_{n}\left(\mathbb{Q}_{p}\right)$, on notera $I(\chi)$ l'induite parabolique normalisée de $P$ à $G$ de la représentation

$$
(\omega \otimes \chi) \times\left(\check{\omega} \otimes \chi^{-1}\right)
$$

de $M\left(\mathbb{Q}_{p}\right)$. Ces représentations seront étudiées en détail dans un paragraphe ultérieur (4.1). Disons simplement ici que les $I(\chi)$ sont irréductibles et autoduales, de sorte qu'elles se prolongent (en fait de manière naturelle car les données ci-dessus sont $\theta$-stables) à $G^{+}\left(\mathbb{Q}_{p}\right)$.

L'objectif principal de ce chapitre est de démontrer le résultat suivant.

Théorème 3.2. Il existe une représentation automorphe cuspidale irréductible $\Pi$ de $\mathrm{GL}_{2 n}(\mathbb{A})$ ayant les propriétés suivantes :

i) $\check{\Pi} \simeq \Pi$,

ii) $\Pi_{\infty}|\cdot|^{(2 n-1) / 2}$ est algébrique régulière,

iii) П est non ramifiée à toutes les places finies différentes de $\ell$ et $p$,

iv) $\Pi_{p} \simeq I(\chi)$ pour un certain $\chi$,

v) $\Pi_{\ell}$ est la représentation de Steinberg.

Afin d'utiliser la formule des traces d'Arthur, nous aurons besoin de certaines propriétés des intégrales orbitales tordues des pseudo-coefficients nécessaires en $\infty, \ell$ et $p$. Le travail archimédien a déjà été fait dans le chapitre précédent. Une première sous-section 3.4 sera consacrée à l'étude des pseudo-coefficients $\theta$-tordus de la représentation de Steinberg, ce qui fournira les renseignements nécessaires en $\ell$. En ce qui concerne la place $p$, nous admettrons au 3.15 le résultat suivant dont la preuve fera l'objet du Chapitre 4. 
Considérons l'elément $\theta$-semisimple elliptique

$$
\gamma_{0}:=\left(\begin{array}{cc}
1_{n} & 0 \\
0 & -1_{n}
\end{array}\right) \in G(\mathbb{Q}) .
$$

Théorème 3.3. Il existe une fonction $f_{p}: G\left(\mathbb{Q}_{p}\right) \longrightarrow \mathbb{C}$ localement constante à support compact ayant les propriétés suivantes:

(i) Si $\pi$ est une représentation lisse irréductible autoduale de $G\left(\mathbb{Q}_{p}\right)$ et si $A$ : $\pi \stackrel{\sim}{\rightarrow} \pi \circ \theta$ est un $G\left(\mathbb{Q}_{p}\right)$-isomorphisme, alors trace $(A \pi(f))=0$ si $\pi$ n'est pas de la forme $I(\chi)$,

(ii) L'intégrale orbitale tordue

$$
\mathrm{TO}_{\gamma_{0}}\left(f_{p}\right)=\int_{I_{\gamma_{0}}\left(\mathbb{Q}_{p}\right) \backslash G\left(\mathbb{Q}_{p}\right)} f_{p}\left(g^{-1} \gamma_{0} \theta(g)\right) \mu
$$

est non nulle 14

3.4. Fonctions d'Euler-Poincaré et représentation de Steinberg dans le cas tordu. Dans cette partie, nous rappelons ou établissons certaines propriétés des fonctions d'Euler-Poincaré associées à un automorphisme d'un groupe réductif connexe. Les énoncés étant ici tout à fait généraux, nous nous plaçons dans tout ce $\$ 3.4$ dans le cadre suivant.

3.4.1. On fixe $F$ un corps local non archimédien de caractéristique nulle, $G$ un groupe réductif connexe sur $F$ et $\theta$ un automorphisme $F$-rationnel de $G$ disons d'ordre fini $h$. On note $G^{+}$le produit semi-direct de $\mathbb{Z} / h \mathbb{Z}$ par $G$ défini par $\theta, S$ le $F$-tore maximal central déployé de $G$ et $X^{*}(S)=\operatorname{Hom}\left(S, \mathbb{G}_{m}\right)$ le groupe abélien libre des caractères rationnels de $S$. Le groupe quotient $G^{+} / G=\langle\theta\rangle$ agit par conjugaison sur $S$; on peut donc considérer le plus grand sous-tore $S^{\theta}$ de $S$ fixé par $\theta$ ainsi que son polynôme caractéristique réciproque

$$
P(z):=\operatorname{det}\left(1-z \theta_{\mid X^{*}(S)}\right) \in \mathbb{Z}[z] .
$$

On définit de plus $q(G) \geq 0$ comme étant le rang d'un $F$-tore déployé maximal du groupe $G / S$.

Nous noterons 1 la représentation triviale. Soit $B$ un parabolique minimal de $G$ défini sur $F$. Notons $I_{B}$ l'espace des fonctions complexes lisses sur $B(F) \backslash G(F)$. C'est une représentation de $G(F)$ par translations à droite dont l'unique quotient irréductible est la représentation de Steinberg de $G(F)$, notée St. Par exemple, St $=1$ si $G$ est un tore. De plus, St se factorise par $G(F) / S(F)=(G / S)(F)$ (via Hilbert 90) : c'est la représentation de Steinberg de $G / S$. La représentation St est de carré intégrable si $S=1$.

Les paraboliques minimaux de $G$ définis sur $F$ étant tous conjugués et égaux à leurs normalisateurs, le $G(F)$-ensemble $B(F) \backslash G(F)$ s'identifie à celui de ces paraboliques minimaux muni de l'action par conjugaison de $G(F)$. Cette dernière s'étend donc canoniquement en une action de $\operatorname{Aut}(G)(F)$, en particulier de $G^{+}(F)$, ce qui fait de $I_{B}$ et St des représentations de $G^{+}(F)$ de manière naturelle (et indépendantes du choix de $B$ ). Il sera commode pour la suite de choisir un élément $g \in G(F)$ tel que $\theta(B)=g^{-1} B g$; alors l'élément

$$
\theta_{B}:=g \theta \in G(F) \theta
$$

\footnotetext{
${ }^{14} \mu$ est ici une mesure $G\left(\mathbb{Q}_{p}\right)$-invariante non nulle quelconque sur $I_{\gamma_{0}}\left(\mathbb{Q}_{p}\right) \backslash G\left(\mathbb{Q}_{p}\right), I_{\gamma_{0}} \simeq \mathrm{Sp}_{2 n}$ étant le centralisateur de $\gamma_{0}$.
} 
satisfait $\theta_{B}(B)=B$. Notons que si $V$ est une représentation de $G^{+}(F)$ dont la restriction à $G(F)$ est isomorphe à St, alors $V \stackrel{\sim}{\rightarrow}$ St $\otimes \chi$ pour un unique caractère $\chi: G^{+}(F) / G(F) \rightarrow \mathbb{C}^{*}$.

Enfin, les $F$-tores déployés maximaux de $B$ étant conjugués sous $B(F)$, on peut supposer quitte à remplacer $g$ par un élément de la forme $b g$ où $b \in B(F)$ que $\theta_{B}$ stabilise un tel tore $S^{\prime}$ de $B$, auquel cas il préserve aussi le centralisateur de $S^{\prime}$ dans $B$ qui est un sous-groupe de Levi de $B$. L'automorphisme $\theta_{B}$ agit sur $\Lambda^{q(G)}\left(X^{*}\left(S^{\prime} / S\right)\right)$ par un signe que l'on note $\epsilon(\theta)$ (il découlera par exemple de la proposition suivante que ce signe ne dépend d'aucun choix).

Exemple 3.5. Dans le cas qui nous intéresse $F=\mathbb{Q}_{l}, G=\mathrm{GL}_{2 n}$ et $\theta$ est comme au 3.1. On a $S=\mathbb{G}_{m}$ et $\theta$ y agit par l'inversion; donc $S^{\theta}=1$ et $P(1)=2$. De plus, $\theta$ préserve le sous-groupe de Borel triangulaire supérieur $B$, son tore diagonal et agit sur ce dernier par $\left(x_{1}, x_{2}, \ldots, x_{2 n}\right) \mapsto\left(x_{2 n}^{-1}, \ldots, x_{2}^{-1}, x_{1}^{-1}\right)$; donc $\varepsilon(\theta)=(-1)^{n-1}$ (et $q(G)=2 n-1)$.

3.5.1. Nombre de Lefschetz d'un automorphisme. Suivant Borel-Wallach BW, X.5], notons $H_{e}^{i}(G(F),-)$ les foncteurs dérivés du foncteur des $G(F)$-invariants de la catégorie des représentations complexes lisses de $G(F)$ dans celle des espaces vectoriels complexes. Il est démontré loc. cit. que si $V$ est une représentation lisse admissible de $G(F)$, alors les $H_{e}^{i}(G(F), V)$ sont de dimension finie, nuls pour $i>q(G)+\operatorname{dim}_{F}(S)$. Si $V$ est de plus la restriction à $G(F)$ d'une représentation de $G^{+}(F)$, ces espaces sont munis d'une action naturelle de $G^{+}(F) / G(F)=\langle\theta\rangle$, ce qui définit le « nombre de Lefschetz » de $\theta$ :

$$
\operatorname{Lef}_{G}(\theta, V)=\sum_{i \geq 0}(-1)^{i} \operatorname{trace}\left(\theta, H_{e}^{i}(G(F), V)\right) .
$$

Ces nombres ont été notamment étudiés par Borel-Labesse-Schwermer BLS, §9] 15 La proposition suivante repose essentiellement sur des résultats de Casselman et Borel-Wallach.

Proposition 3.6. Lef $(\theta, \cdot)$ est non identiquement nul si, et seulement si, $S^{\theta}=\{1\}$, ou ce qui est équivalent si $P(1) \neq 0$. Supposons donc que $P(1) \neq 0$ et fixons $V$ une représentation irréductible unitaire de $G^{+}(F)$.

(i) $\operatorname{Lef}_{G}(\theta, 1)=P(1)$ et $\operatorname{Lef}_{G}(\theta, \mathrm{St})=P(1)(-1)^{q(G)} \varepsilon(\theta)$.

(ii) Si $V$ est essentiellement tempérée, $\operatorname{Lef}_{G}(\theta, V) \neq 0$ si, et seulement si, $V_{\mid G(F)}=$ St.

(iii) Si $G / S$ est quasi-simple, $\operatorname{Lef}_{G}(\theta, V) \neq 0$ si, et seulement si, $V_{\mid G(F)} \in$ $\{1, \mathrm{St}\}$.

Preuve. Soit $V$ une représentation complexe lisse de $G(F)$. D'après Hitta [Hi], on dispose pour la paire $S(F) \subset G(F)$ d'une suite spectrale de type Hochschild-Serre

$$
E_{2}^{p, q}=H_{e}^{p}\left(G(F) / S(F), H_{e}^{q}(S(F), V)\right) \Longrightarrow H_{e}^{p+q}(G(F), V) .
$$

Si $V$ est la restriction à $G(F)$ d'une représentation de $G^{+}(F)$, cette suite spectrale est par construction naturellement munie d'une action de $G^{+}(F) / G(F)$ compatible

\footnotetext{
${ }^{15}$ Notons que la Proposition $8.2(2)$ de $\left[\right.$ BLS] est incorrecte : si $S^{\theta} \neq 1$, alors $\operatorname{Lef}_{G}(\theta,-)$ est identiquement nul, ce qu'ils démontrent d'ailleurs au passage au cours de la preuve de leur Proposition 8.4. L'erreur se trouve dans l'appel à [BW, X.4.7], qui nécessite $G$ simplement connexe.
} 
à l'action rappelée plus haut sur son aboutissement. Si de plus $S(F)$ agit trivialement sur $V, E_{2}^{p, q}$ s'écrit alors $H_{e}^{q}(S(F), 1) \otimes_{\mathbb{C}} H_{e}^{p}(G(F) / S(F), V)$. Dans le cas où $V$ est de surcroît admissible on obtient donc l'identité

$$
\operatorname{Lef}_{G}(\theta, V)=\operatorname{Lef}_{S}(\theta, 1) \operatorname{Lef}_{G / S}(\theta, V) .
$$

Supposons maintenant que $W$ est une représentation lisse irréductible de $G(F)$ telle que $H_{e}^{i}(G(F), W) \neq 0$ pour un certain entier $i \geq 0$. D'après un résultat de Borel-Casselman, $W$ est un constituant irréductible de $I_{B}$ ([BW, X.2.4, X.4.3]). En particulier, $W$ est de caractère central trivial et elle se factorise donc en une représentation de $G(F) / S(F)=(G / S)(F)$. Ainsi, d'après l'identité (3.1), il suffit de démontrer la proposition dans les cas $S=\{1\}$ et $G=S$ (et dans ce cas pour $V=1)$.

Supposons d'abord $G=S$. La valuation $F^{*} \longrightarrow \mathbb{Z}$ induit un isomorphisme canonique $X^{*}(S) \otimes_{\mathbb{Z}} \mathbb{C} \stackrel{\sim}{\rightarrow} \operatorname{Hom}_{\mathbb{Z}}\left(S(F) / S(F)^{0}, \mathbb{C}\right)$, où $S(F)^{0}$ est le sous-groupe compact maximal de $S(F)$. Le calcul standard de la cohomologie des tores nous fournit alors des isomorphismes canoniques ([BW, X.2.6])

$$
H_{e}^{i}(S(F), 1) \stackrel{\sim}{\rightarrow} \Lambda^{i}\left(X^{*}(S) \otimes_{\mathbb{Z}} \mathbb{C}\right), \quad i \geq 0,
$$

d'où l'on tire $\operatorname{Lef}_{S}(\theta, 1)=P(1)$.

Supposons enfin $S=\{1\}$ (i.e., $G(F)$ de centre compact) et fixons $V$ comme dans l'énoncé. D'après Borel-Wallach [BW, XI.3.8], si $V$ est tempérée et si $H_{e}^{i}(G(F), V) \neq$ 0 , alors $i=q$ et $V_{\mid \mathrm{G}(F)}=$ St. De plus, si $H_{e}^{i}(G(F), 1) \neq 0$, alors $i=0$. Enfin, si $G$ est quasi-simple et si $H_{e}^{i}(G(F), V) \neq 0$, alors $V_{\mid G(F)} \in\{1$,St $\}$ d'après [BW, XI.3.9]. Il ne reste donc qu'à verifier que $\theta$ agit sur $H_{e}^{d(G)}(G(F)$, St) par la multiplication par le signe $\epsilon(\theta)$ défini au 3.4.1. Comme les $H_{e}^{i}(G(F), \cdot)$ s'annulent si $i>q(G)$, on dispose d'une surjection

$$
H_{e}^{q(G)}\left(G(F), I_{B}\right) \longrightarrow H_{e}^{q(G)}(G(F), \mathrm{St})
$$

et il suffit de voir que l'espace de gauche est de dimension 1 et de montrer que l'action de $\theta$, ou ce qui est équivalent de $\theta_{B}$, y agit par $\varepsilon(\theta)$. Par le lemme de Shapiro ([BW, X.4.2]) appliqué à la représentation induite $I_{B}$, on dispose d'une identification $\theta_{B}$-équivariante $H_{e}^{q(G)}\left(G(F), I_{B}\right) \stackrel{\sim}{\rightarrow} H_{e}^{q(G)}(M(F), 1)$ où $M$ est le Levi de $B$ centralisant $S^{\prime}$. Comme $M(F) / S^{\prime}(F)=\left(M / S^{\prime}\right)(F)$ est compact, la suite spectrale de Horschild-Serre rappelée plus haut fournit pour tout $i \geq 0$ un isomorphisme canonique $\theta_{B}$-équivariant $H_{e}^{i}\left(S^{\prime}(F), 1\right) \stackrel{\sim}{\rightarrow} H_{e}^{i}(M(F), 1)$, et on conclut alors par l'identité (3.2) appliquée à $G=S^{\prime}(F)$ et $i=q(G)$.

Remarque 3.7. Soient $P$ un sous-groupe parabolique de $G$ et $M$ un sous-groupe de Levi de $P$ qui sont tous deux définis sur $F$ et normalisés par un élément $\theta_{M} \in$ $G(F) \theta$. Par le lemme de Shapiro lisse, pour toute représentation admissible $W$ de $M(F) \rtimes\left\langle\theta_{M}\right\rangle$, on a

$$
\operatorname{Lef}_{G}\left(\theta, \operatorname{Ind}_{P}^{G} W\right)=\operatorname{Lef}_{M}\left(\theta_{M}, W \delta_{P}^{1 / 2}\right)
$$

(l'induite de $P(F)$ à $G(F)$ étant lisse et normalisée). En particulier, ce nombre est nul si $W$ est unitaire et $P \neq G$, car les constituants irréductibles de $W \delta_{P}^{1 / 2}$ sont de caractère central non trivial (et même non unitaire). 
3.7.1. Fonctions d'Euler-Poincaré d'un automorphisme. Fixons une mesure de Haar $\mu$ sur $G^{+}(F)$.

Proposition 3.8. Supposons $P(1) \neq 0$. Il existe une function $f_{E P}: G^{+}(F) \longrightarrow \mathbb{C}$ localement constante et à support compact dans $\theta G(F)$ telle que pour toute representation admissible $(\pi, V)$ de $G^{+}(F)$ on ait $\operatorname{trace}\left(\pi\left(f_{E P}\right)\right)=\operatorname{Lef}_{G}(\theta, V)$. Elle jouit de plus des propriétés suivantes:

(i) $f_{E P}^{\prime}:=\frac{(-1)^{q(G)} \epsilon(\theta)}{P(1)} f_{E P}$ est un pseudo-coefficient de $\mathrm{St}: \operatorname{trace}\left(\operatorname{St}\left(f_{E P}^{\prime}\right)\right)=1$ et si $\pi$ est irréductible essentiellement tempérée telle que $\pi_{\mid G(F)} \neq \mathrm{St}$, alors $\operatorname{trace}\left(\pi\left(f_{E P}^{\prime}\right)\right)=0$.

(ii) Soient $\gamma \in \theta G(F)$ un élément semisimple, $I_{\gamma}$ la composante neutre du centralisateur de $\gamma$ dans $G$; on munit $I_{\gamma}(F) \backslash G(F)$ d'une mesure $G(F)$-invariante $\bar{\mu}$. Alors l'intégrale orbitale "tordue»

$$
O_{\gamma}\left(f_{E P}\right):=\int_{I_{\gamma}(F) \backslash G(F)} f_{E P}\left(g^{-1} \gamma g\right) \bar{\mu}
$$

est non nulle si et seulement si $I_{\gamma}(F)$ est de centre compact.

Dans le cas où $G / S$ est quasi-simple, (i) admet la variante plus forte

(i) Si $G / S$ est quasi-simple et $V_{\mid G(F)} \neq \mathrm{St}, 1$ est irréductible et unitaire, alors $\operatorname{trace}\left(\pi\left(f_{E P}\right)\right)=0$.

Remarque 3.9. Ces fonctions $f_{E P}$ ont été introduites par Kottwitz dans [K1, §2] sous le nom de fonctions d'Euler-Poincaré dans le cas où $S$ et $\theta$ sont triviaux; il y démontre la proposition dans ce cadre. Certaines de leurs propriétés ont été étendues par Borel-Labesse-Schwermer dans [BLS] dans la généralité adoptée ici (voir cependant les notes de bas de page 17 et 18). Les arguments de cette section ne sont que des adaptations essentiellement triviales de ces résultats. Notons que l'existence d'une fonction $f_{E P}$ satisfaisant (i) pourrait se déduire du théorème de Paley-Wiener, mais la démonstration de Kottwitz a l'avantage de fournir une fonction $f_{E P}$ explicite en terme de l'immeuble de Bruhat-Tits de $G$ et de fournir la propriété (ii) à peu de frais.

Le reste de cette partie sera consacré à la preuve de la proposition.

3.9.1. L'immeuble de Bruhat-Tits de $G$ et définition de $f_{E P}$. Soit $\mathcal{B}$ l'immeuble de Bruhat-Tits de $G$ ([Ti]). C'est un complexe polysimplicial ([BT, §1.1]) muni d'une action simpliciale de $G(F)$, et même de $G^{+}(F)$. Le centre de $G(F)$ n'étant pas nécessairement compact, il convient de préciser notre convention concernant la partie torique de $\mathcal{B}$ (ce n'est pas celle de [Ti, §1]; en revanche, c'est celle adoptée dans BW, Chap. X, §2.3]).

Tout d'abord, si le groupe $G$ est semisimple, l'immeuble $\mathcal{B}$ est celui décrit dans Ti, §2]. Il est muni d'une structure polysimpliciale canonique, et d'une action naturelle de $\operatorname{Aut}(G)(F)([\mathrm{Ti}, \S 2.5])$. Si à l'inverse $G$ est un tore, et si $G(F)^{0} \subset G(F)$ désigne l'unique sous-groupe compact maximal, alors $G(F) / G(F)^{0}$ est un groupe abélien libre de type fini et nous posons

$$
\mathcal{B}=\left(G(F) / G(F)^{0}\right) \otimes_{\mathbb{Z}} \mathbb{R} .
$$

L'action par translations de $G(F)$ sur $G(F) / G(F)^{0}$ s'étend à $G^{+}(F)$ si l'on pose $\theta\left(g G(F)^{0}\right)=\theta(g) G(F)^{0}$, ce qui munit $\mathcal{B}$ d'une action affine de $G^{+}(F)$. On choisit 
alors une décomposition simpliciale de $\mathcal{B}$ invariante 16 par $G^{+}(F)$. Enfin, quand $G$ est réductif général, on pose

$$
\mathcal{B}=\mathcal{B}_{1} \times \mathcal{B}_{2}
$$

où $\mathcal{B}_{1}$ est l'immeuble du groupe adjoint $G_{\text {ad }}$ et $\mathcal{B}_{2}$ celui du cocentre $G / G_{\text {der }}$, où l'on fixe sur $\mathcal{B}_{2}$ une structure simpliciale invariante par $\left(G^{+} / G_{\text {der }}\right)(F)$. Le groupe $G^{+}(F)$ agit alors sur $\mathcal{B}_{1}$ et $\mathcal{B}_{2}$ via les morphismes naturels $G^{+}(F) \rightarrow \operatorname{Aut}\left(G_{\text {ad }}\right)(F)$ et $G^{+}(F) \rightarrow\left(G^{+} / G_{\text {der }}\right)(F)$, puis sur $\mathcal{B}$ par l'action diagonale.

Rappelons qu'un sommet de $\mathcal{B}$ est un polysimplexe de dimension 0 et qu'une chambre de $\mathcal{B}$ est l'intérieur d'un polysimplexe de dimension maximale, en l'occurence $\operatorname{dim}(S)+q(G)$. L'immeuble $\mathcal{B}$ est contractile et a la propriété que le stabilisateur dans $G(F)$ de chaque partie compacte de $\mathcal{B}$ est un sous-groupe compact ouvert, de sorte que le complexe de ses chaines polysimpliciales orientées permet de calculer la cohomologie des représentations lisses de $G(F)$ (Casselman-Wigner, Borel-Wallach [BW, X.2]) de la manière suivante.

Rappelons qu'un polysimplexe orienté est la donnée d'un couple $(p, \epsilon)$ où $p$ est un polysimplexe et $\epsilon$ une orientation de $p$ (égale à 1 par convention si $p$ est de dimension 0 ). Pour $i \geq 0$ notons $\mathcal{P}_{i, \pm}$ l'ensemble des polysimplexes orientés de $\mathcal{B}$ de dimension $i$, il est muni d'une action naturelle de $G^{+}(F)$. Si $V$ est une représentation lisse de $G(F)$ et $i \geq 0$, définissons $C_{i}(V)$ comme étant l'espace des fonctions $G(F)$-équivariantes $\varphi: \mathcal{B}_{i, \pm} \longrightarrow V$ telles que $\varphi((p,-\varepsilon))=-\varphi(p, \varepsilon)$ pour tout polysimplexe orienté $(p, \varepsilon) \in \mathcal{B}_{i, \pm}$ de dimension $>0$. Les $C:=\left(C_{i}\right)_{i \geq 0}$ forment alors un complexe de cochaînes dont la cohomologie est $H_{e}^{i}(G(F), V)$. On suppose dorénavant que $V$ est une représentation admissible de $G^{+}(F), G^{+}(F) / G(F)$ agit alors naturellement sur $C$ par la formule $\theta(f)(x)=\theta\left(f\left(\theta^{-1}(x)\right)\right), \forall f \in C_{i}(V)$.

D'après Bruhat-Tits, l'action de $G(F)$ sur les polysimplexes n'a qu'un ensemble fini $\Sigma$ d'orbites. Si $s$ est une telle orbite, on note $\operatorname{dim}(s)$ la dimension commune de ses polysimplexes, et on note $\Sigma_{i} \subset \Sigma$ le sous-ensemble des orbites de dimension $i$. Il vient que l'on a une décomposition $G(F)$-équivariante $C_{i}(V)=\bigoplus_{s \in \Sigma_{i}} C_{i}(V)_{s}$, où $C_{i}(V)_{s}$ est le sous-espace des fonctions à support dans l'orbite $s$.

Si $p$ est un polysimplexe, notons $G_{p}$ (resp. $G_{p}^{+}$) le sous-groupe de $G(F)$ (resp. $G^{+}(F)$ ) laissant $p$ globalement invariant : c'est un sous-groupe compact ouvert. On dispose alors d'un caractère d'orientation 17 continu $\operatorname{sign}_{p}: G_{p}^{+} \rightarrow\{ \pm 1\}$ et on note $V_{p}$ le plus grand sous-espace de $V$ sur lequel $G_{p}$ agit par $\operatorname{sign}_{p}$. Si $p \in s, \phi \mapsto \phi(p)$ induit alors un isomorphisme $C_{i}(V)_{s} \stackrel{\sim}{\rightarrow} V_{p}$ (qui est donc de dimension finie). Il y a deux cas :

- Si $\theta(s) \neq s$, alors trace $\left(\theta, \sum_{n \in \mathbb{Z}} C_{i}(V)_{\theta^{n}(s)}\right)=0$.

- Si $\theta(s)=s$, il existe $\theta_{s} \in G(F) \theta$ tel que $\theta_{s}(p)=p$. La trace de $\theta$ sur $C_{i}(V)_{s}$ ne dépendant que de l'image de $\theta$ dans $G^{+}(F) / G(F)$, on peut la calculer pour $\theta_{s}$ par évaluation en $p$, et l'on trouve $\operatorname{sign}_{p}\left(\theta_{s}\right) \operatorname{trace}\left(\theta_{s}, V_{p}\right)$ (noter que $G_{p}^{+}=\left\langle G_{p}, \theta_{s}\right\rangle$ et $\theta_{s}$ normalise $\left.G_{p}\right)$.

\footnotetext{
${ }^{16}$ Par exemple, choisir un produit scalaire $G^{+}(F)$-invariant sur $\mathcal{B}$ et considérer la subdivision barycentrique d'un domaine fondamental de Dirichlet pour l'action de $G^{+}(F)$.

${ }^{17} \mathrm{Si} p$ est un simplexe et $g \in G_{p}^{+}, \operatorname{sign}_{p}(g)$ est aussi la signature de la permutation des sommets de $p$ induite par $g$. Contrairement à ce qui semble sous-entendu dans [K1, §2] et dans la preuve de [BLS, Prop. 8.4], précisons que cela ne vaut plus pour un polysimplexe général : considérer une réflexion d'un carré d'axe passant par le milieu d'un coté (cela se produit par exemple si $\left.G=G^{+}=P G L_{2} \times P G L_{2}\right)$.
} 
Pour chaque $s \in \Sigma$ fixé par $\theta$, on choisit alors un $p_{s} \in s$ et on définit $f_{s}$ comme étant la fonction sur $G^{+}(F)$ qui vaut $\operatorname{sign}_{p}$ sur $G_{p}^{+} \cap \theta G(F)$ et qui est nulle ailleurs. Si l'on pose 18

$$
f_{E P}:=\sum_{s \in \Sigma, \theta(s)=s}(-1)^{\operatorname{dim}(s)} \mu\left(G_{p_{s}}\right)^{-1} f_{s},
$$

on a montré que trace $\left(\pi\left(f_{E P}\right), V\right)=\operatorname{Lef}_{G}(\theta, V)$, i.e. que $f_{E P}$ est une fonction d'Euler-Poincaré pour $\theta$. Le point (i) de la proposition découle alors de la Proposition 3.8 . Notons que par construction, la mesure $\mu f_{E P}$ est indépendante du choix de $\mu$, et que les intégrales orbitales des $f_{s}$, et donc de $f_{E P}$, ne dépendent pas du choix des $p_{s}$.

3.9.2. Intégrales orbitales de $f_{E P}$ en les éléments semisimples de $G(F) \theta$. Il ne reste qu'à démontrer (ii). La démonstration de Kottwitz [K1, Theorem 2.2] s'étend essentiellement verbatim. Faisons agir $G(F)$ sur $G(F)^{+}$par la conjugaison « à droite » $g \cdot \gamma:=g^{-1} \gamma g$ et fixons $\gamma \in G(F) \theta$ un élément de $G(F)^{+}$comme dans l'énoncé. Comme $\gamma$ est semisimple, l'orbite $\omega(\gamma):=G(F) \cdot \gamma$ est alors fermée dans $G(F) \theta$ et l'intégrale orbitale de l'énoncé est trivialement convergente.

Le sous-espace $\mathcal{B}^{\gamma} \subset \mathcal{B}$ des points fixes de $\gamma$ est muni d'une structure de complexe polysimplicial dont les polysimplexes sont les $p \cap \mathcal{B}^{\gamma}$ non vides, i.e. tels que $p$ est dans l'ensemble $\mathcal{F}(\gamma)$ des polysimplexes de $\mathcal{B}$ stables globalement par $\gamma$. Supposons que $\mathcal{B}^{\gamma}$ est non vide, ce qui est toujours le cas si le centre de $I_{\gamma}(F)$ est compact 19 C'est alors un ensemble clos au sens de Bruhat-Tits, il est en particulier contractile. Il hérite de $(\mathcal{B}, G(F))$ les propriétés suivantes : $I_{\gamma}(F)$ agit de manière cellulaire sur $\mathcal{B}^{\gamma}$, tout point de $\mathcal{B}^{\gamma}$ a un stabilisateur compact ouvert, et tout sous-groupe compact de $I_{\gamma}$ fixe un point de $\mathcal{B}^{\gamma}$. Enfin, si $p$ est un polysimplexe de $\mathcal{B}$ et $g \in G(F)$,

$$
g . \gamma \in G_{p}^{+} \Leftrightarrow g p \in \mathcal{F}(\gamma),
$$

ce qui ne dépend que de $I_{\gamma}(F) g G_{p}$. En particulier, $I_{\gamma}(F)$ n'a qu'un nombre fini d'orbites sur $G(F) p \cap \mathcal{F}(\gamma)$, et donc sur $\mathcal{F}(\gamma)$, car $\omega(\gamma) \cap G_{p}^{+}$est compact. D'après Serre [S1, §3.3], toutes les conditions sont donc satisfaites pour que le complexe cellulaire $B^{\gamma}$ permette de calculer la mesure d'Euler-Poincaré de $I_{\gamma}$ : si $\mu_{I}$ est la mesure de Haar sur $I_{\gamma}$ telle que $\bar{\mu}=d \mu / d \mu_{I}$, c'est la mesure signée canonique

$$
\mu_{E P, I_{\gamma}}:=\sum_{p \in I_{\gamma}(F) \backslash \mathcal{F}(\gamma)}(-1)^{\operatorname{dim}(p)} \frac{\mu_{I}}{\mu_{I}\left(I_{\gamma}(F) \cap G_{p}\right)} .
$$

D'après [S1, Prop. 28], cette mesure est non nulle si, et seulement si, $I_{\gamma}(F)$ est de centre compact; son signe est alors $(-1)^{q\left(I_{\gamma}\right)}$.

Il ne reste qu'à vérifier que $O_{\gamma}\left(f_{E P}\right) \mu_{I}=\mu_{E P, I_{\gamma}}$ si $\mathcal{B}^{\gamma} \neq \emptyset$ et qu'elle est nulle sinon. Notons d'abord que si $\theta(s) \neq s, s \cap \mathcal{F}(\gamma)=\emptyset$. Soit $s=G(F) p_{s} \in \Sigma^{\theta}$; alors

\footnotetext{
${ }^{18}$ Noter que strictement, l'expression de $f_{E P}$ donnée par Borel-Labesse-Schwermer dans la preuve de BLS Prop. 8.4] n'est valable que quand $G$ est semisimple et simplement connexe, qui est l'hypothèse de validité de [BW X.2.5] auquel ils renvoient, et auquel cas le complexe $C$ se simplifie. C'est une des raisons pour laquelle nous avons redonné l'argument complet.

${ }^{19} \mathrm{En}$ effet, une puissance finie de $\gamma$ est dans le centre de $I_{\gamma}(F)$; elle admet donc un point fixe $x \in B^{\gamma}$ quand ce centre est compact. L'ensemble fini $\left\{\gamma^{n} x, n \in \mathbb{Z}\right\} \subset \mathcal{B}$ est alors stable par $\gamma$ et son enveloppe convexe contient un élément de $\mathcal{B}^{\gamma}$ d'après [BT] Prop. 3.2.4].
} 
par définition de $f_{s}$ et (3.4) on a

$$
\mathrm{O}_{\gamma}\left(f_{s}\right)=\sum_{p \in I_{\gamma}(F) \backslash(s \cap \mathcal{F}(\gamma))} \operatorname{sign}_{p}(\gamma) \mu_{I}\left(I_{\gamma}(F) \cap G_{p}\right)^{-1} .
$$

On conclut en notant que 20 si $p \in \mathcal{F}(\gamma), \operatorname{sign}_{p}(\gamma)=(-1)^{\operatorname{dim}(p)-\operatorname{dim}\left(p \cap \mathcal{B}^{\gamma}\right)}$.

Terminons par un corollaire important de cette démonstration, précisant la Proposition 3.8 (ii).

Corollaire 3.10. Supposons que $\gamma \in \theta G(F)$ est semisimple et que $I_{\gamma}(F)$ est de centre compact. Si l'on munit $I_{\gamma}(F)$ de sa mesure d'Euler-Poincaré di, et si $\bar{\mu}=\frac{d \mu}{d i}$, alors

$$
O_{\gamma}\left(f_{E P}\right)=1
$$

3.10.1. Intégrales orbitales non semisimples. Un élément semisimple $\gamma \in \theta G(F)$ est dit elliptique si $I_{\gamma}(F)$ est de centre compact.

Proposition 3.11. Soit $f \in \mathcal{C}_{c}^{\infty}(\theta G(F))$ telle que $\mathrm{O}_{\gamma}(f)=0$ pour tout élément $\gamma \in$ $\theta G(F)$ semisimple non elliptique, et que $\mathrm{O}_{\gamma}(f)=1$ si $\gamma \in \theta G(F)$ est semisimple, elliptique et régulier, la mesure sur son centralisateur étant la mesure d'EulerPoincaré. Alors $\mathrm{O}_{\gamma}(f)=0$ pour tout élément $\gamma \in \theta G(F)$ non semisimple.

Preuve. En effet, écrivons

$$
\gamma=\nu \sigma=\sigma \nu
$$

la décomposition de Jordan de $\gamma$, où $\nu \in G(F)$ est unipotent non trivial et $\sigma \in$ $\theta G(F)$ semisimple. Si $I=I_{\gamma}$ et $M=I_{\sigma}$ désignent les centralisateurs connexes respectifs de $\gamma$ et $\sigma$ dans $G$, alors $M$ est réductif, $I$ est unimodulaire et $I \subset M$. De plus, $\nu \in M(F)$. Si $f \in \mathcal{C}_{c}^{\infty}(\theta G(F))$, on peut écrire

$$
\begin{gathered}
\mathrm{O}_{\gamma}(f)=\int_{I(F) \backslash G(F)} f\left(g^{-1} \gamma g\right) \frac{d g}{d i} \\
=\int_{M(F) \backslash G(F)} \int_{I(F) \backslash M(F)} f\left(g^{-1} m^{-1} \nu \sigma m g\right) \frac{d m}{d i} \frac{d g}{d m} \\
=\int_{M(F) \backslash G(F)} \int_{I(F) \backslash M(F)} f\left(g^{-1} m^{-1} \nu m \sigma g\right) \frac{d m}{d i} \frac{d g}{d m},
\end{gathered}
$$

la seconde intégrale étant l'intégrale orbitale ordinaire dans le groupe réductif connexe $M$ de la fonction

$$
f_{g}^{M}(m):=f\left(g^{-1} m \sigma g\right)
$$

en $\nu \in M(F)$ (remarquer que le centralisateur de $\nu$ dans $M$ est exactement $I$ ).

D'après le lemme de compacité usuel, étendu par Arthur [A3, Lemme 2.1] au cas tordu, il existe un voisinage ouvert $M(F)$-invariant $\mathcal{U}$ de 1 dans $M(F)$ tel que, pour tout sous-ensemble compact $\Omega$ de $\theta G(F)$, il existe un sous-ensemble compact $\omega$ de $M(F) \backslash G(F)$ ayant la propriété suivante : si $g \in G(F)$ et $g^{-1} \mathcal{U} \sigma g \cap \Omega \neq \emptyset$, alors $M(F) g \in \omega$. Soit $M^{+}$le centralisateur de $\sigma$ dans $G$.

Lemme 3.12. Il existe un voisinage ouvert compact $\mathcal{V}$ de 1 dans $M(F)$ ayant la propriété suivante : pour tous $u \in \mathcal{V}$ et $g \in G(F)$ tels que $g^{-1} u \sigma g \in \sigma \mathcal{V}$, alors $g \in M^{+}(F)$.

\footnotetext{
${ }^{20}$ Se ramener à vérifier cette relation pour le déterminant d'une isométrie d'un espace affine euclidien ayant un point fixe.
} 
Preuve. Il s'agit d'une variante de [La1, Lemme 3.1.4]. D'après [La1, Lemme 3.1.1] 21 il existe une sous-variété analytique $Y=Y^{-1} \subset G(F)$ et un voisinage ouvert $\mathcal{V}$ de 1 dans $M(F)$ tels que l'application

$$
Y \times \mathcal{V} \longrightarrow \sigma G(F), \quad(y, u) \mapsto y^{-1} u \sigma y,
$$

soit un difféomorphisme sur son image $\Omega$, un voisinage ouvert compact de $\sigma$ dans $\sigma G(F)$. De plus, si $(y, u) \in Y \times M(F)$ est tel que $y^{-1} u \sigma y \in \sigma \mathcal{V}$, alors $u \in \mathcal{V}$ et $y=1$. En particulier,

$$
\forall(g, u) \in\left(M^{+}(F) Y\right) \times \mathcal{V}, g^{-1} u \sigma g \in \sigma \mathcal{V} \Longrightarrow g \in M^{+}(F) .
$$

Supposons maintenant par l'absurde que l'énoncé ne tient pas : il existe une suite d'éléments $\left(g_{n}, u_{n}, v_{n}\right)$ de $\left(G(F) \backslash M^{+}(F)\right) \times \mathcal{V} \times \mathcal{V}$ telle que $\left(u_{n}, v_{n}\right) \rightarrow(1,1)$ et $g_{n}^{-1} u_{n} \sigma g_{n}=v_{n} \sigma$. D'après (3.5), $g_{n} \notin M^{+}(F) Y$. D'après le lemme de compacité, on peut trouver un compact $\omega \subset G(F)$ tel que $\omega \cap M^{+}(F)=\emptyset$ et tel que pour tout $n$ assez grand, $g_{n}=m_{n} w_{n}$ avec $w_{n} \in \omega$ et $m_{n} \in M^{+}(F)$. On peut donc supposer que $w_{n} \rightarrow w \in \omega$, auquel cas $m_{n}^{-1} u_{n} m_{n}$ converge, disons vers $u^{*} \in M(F)$, qui est nécessairement unipotent car $u_{n} \rightarrow 1$. De plus,

$$
w^{-1} u^{*} \sigma w=\sigma
$$

de sorte que $u^{*}=1$ par unicité de la décomposition de Jordan de $\sigma$, puis $w \in$ $M^{+}(F)$, ce qui est absurde.

Si $u \in M(F)$, considérons le centralisateur connexe $I_{u \sigma}$ de $u \sigma$ dans $G$. D'après le Lemme 3.12 on peut trouver un voisinage ouvert $\mathcal{V}$ de 1 dans $M(F)$ tel que pour tout $u \in \mathcal{V}, I_{u \sigma} \subset M$. Quitte à remplacer $\mathcal{V}$ par $\left(\bigcup_{m \in M(F)} m^{-1} \mathcal{V} m\right) \cap \mathcal{U}$ on peut supposer que $\mathcal{V}$ est $M(F)$-invariant et inclus dans $\mathcal{U}$. Noter que $\mathcal{V}$ (tout comme $\mathcal{U}$ ) contient alors tous les éléments unipotents de $M(F)$.

Soit $\Omega$ le support de $f$, le lemme de compacité lui associe un $\omega$ que l'on peut supposer ouvert compact. Soient $\chi \in \mathcal{C}_{c}^{\infty}(M(F) \backslash G(F))$ la fonction caractéristique de $\omega, \alpha \in \mathcal{C}_{c}^{\infty}(G(F))$ telle que $\int_{M(F)} \alpha(m g) d m=\chi(M(F) g)$, et soit

$$
h(m)=\int_{G(F)} \alpha(g) f_{g}^{M}(m) d g .
$$

Il est clair que $h$ est localement constante sur $M(F)$; son support est compact car si $h(m) \neq 0, m \sigma \in \operatorname{Supp}(\alpha) \operatorname{Supp}(f) \operatorname{Supp}(\alpha)^{-1}$. Si $u \in \mathcal{V}$, alors $I_{u \sigma} \subset M$ et on montre comme plus haut que

$$
\mathrm{O}_{u \sigma}(f)=\int_{M(F) \backslash G(F)} \mathrm{O}_{u}^{M}\left(f_{g}^{M}\right) \frac{d g}{d m}=\int_{M(F) \backslash G(F)} \chi(g M) \mathrm{O}_{u}^{M}\left(f_{g}^{M}\right) \frac{d g}{d m},
$$

cette dernière égalité venant de ce que $\mathcal{V} \subset \mathcal{U}$, puis

$$
\mathrm{O}_{u \sigma}(f)=\int_{G(F)} \alpha(g) \mathrm{O}_{u}^{M}\left(f_{g}^{M}\right) d g=\mathrm{O}_{u}^{M}(h) .
$$

Par conséquent, pour $u \in \mathcal{V}, \mathrm{O}_{u \sigma}(f)$ est une intégrale orbitale ordinaire sur le groupe $M$.

Si $M$ contient un tore maximal non compact $T$, alors

$$
\mathrm{O}_{u}^{M}(h)=\mathrm{O}_{u \sigma}(f)=0
$$

\footnotetext{
${ }^{21}$ Bien que le cadre adopté loc. cit. soit celui du changement de base, la démonstration de Labesse s'applique verbatim au cas général.
} 
pour tout élément régulier $u \sigma$ dans $(T(F) \cap \mathcal{V}) \sigma$ d'après (3.6) et par hypothèse (ces $u \sigma$ sont semisimples non elliptiques). Si au contraire $T$ est compact, $\mathrm{O}_{u}^{M}(h)$ est constante pour un élément régulier $u \in T(F) \cap \mathcal{V}$. L'argument de Kottwitz [K1, p. 636] montre alors que les intégrales orbitales de $h$ en les éléments unipotents non triviaux s'annulent. En particulier,

$$
\mathrm{O}_{\nu}^{M}(h)=\mathrm{O}_{\gamma}(f)=0 .
$$

Remarque 3.13. Ce résultat est supposé « bien connu », mais n'est apparemment démontré nulle part.

La proposition 3.8 (ii) admet le corollaire suivant :

Corollaire 3.14. Soit $\gamma \in \theta G(F)$ non semisimple elliptique; alors $\mathrm{O}_{\gamma}\left(f_{E P}\right)=0$.

3.15. Preuve du Théorème 3.2, Replaçons-nous sous les hypothèses du $\$ 3.1$

3.15.1. Une version simplifiée de la formule des traces d'Arthur. Soit $A=\mathbb{R}_{+}^{*}$ la composante neutre topologique du centre de $\mathrm{GL}_{2 n}(\mathbb{R})$, équipons l'espace homogène $A \mathrm{GL}_{2 n}(\mathbb{Q}) \backslash \mathrm{GL}_{2 n}(\mathbb{A})$ d'une mesure (finie) $G(\mathbb{A})$-invariante à droite. La représentation unitaire $R$ de $G(\mathbb{A})$ par translations à droite sur l'espace des fonctions cuspidales

$$
L_{\text {cusp }}^{2}\left(A \mathrm{GL}_{2 n}(\mathbb{Q}) \backslash \mathrm{GL}_{2 n}(\mathbb{A})\right)
$$

s'étend en une représentation unitaire de $G^{+}(\mathbb{A})$ si l'on fait agir $\theta$ par l'opérateur $I_{\theta}(\varphi)(x)=\varphi(\theta(x))$. Cette représentation est discrète. Si $f=f_{\infty} \otimes f^{\infty}$ est dans $\mathcal{C}_{c}^{\infty}(G(\mathbb{A}))$, avec $f_{\infty}$ disons $\mathrm{SO}_{2 \mathrm{n}}(\mathbb{R})$-finie, alors $R(f) I_{\theta}$ est traçable et

$$
\operatorname{trace}\left(R(f) I_{\theta}\right)=\sum_{\Pi} \operatorname{trace}\left(R(f) I_{\theta}, \Pi\right),
$$

la somme ci-dessus portant sur les représentations automorphes cuspidales irréductibles et autoduales de $G(\mathbb{A})$. Ces traces dépendent toutes d'un choix de mesure de Haar adélique $d g_{\mathbb{A}}$ sur $G(\mathbb{A})$ que nous fixons une fois pour toutes.

Pour un choix de fonctions tests $f$ convenables les résultats d'Arthur donnent une expression géométrique simple de trace $\left(R(f) I_{\theta}\right)$. Écrivons pour cela $f=f_{\infty} \otimes$ $f_{\ell} \otimes f_{p} \otimes f^{\infty, \ell, p}$ où :

- $f_{\infty}$ est un pseudo-coefficient tordu d'une série $\theta$-discrète cohomologique (cf. $\$ 2.192 .7$.

- $f_{\ell}$ est une fonction d'Euler-Poincaré fixée pour l'automorphisme $\theta$ de $G_{\mathbb{Q}_{l}}$ donnée par la Proposition 3.8 (cf. Exemple 3.5).

- $f_{p}$ est une fonction donnée par le Théorème 3.3 .

- $f^{\infty, \ell, p}$ est la fonction caractéristique de $\prod_{v \notin\{\infty, \ell, p\}} \mathrm{GL}_{2 n}\left(\mathbb{Z}_{v}\right)$.

Rappelons qu'un élément $\theta$-semisimple $\gamma \in \mathrm{G}(\mathbb{Q})$ est dit elliptique si la composante déployée du centre de son centralisateur tordu est triviale. Notons $\{G(\mathbb{Q})\}_{\text {ell }}$ l'ensemble des classes de $\theta$-conjugaison d'éléments $\theta$-semisimples elliptiques. Pour $\gamma \in G(\mathbb{Q})$ un tel élément, on choisit une mesure de Haar adélique $d i_{\mathbb{A}}$ sur $I_{\gamma}(\mathbb{A})$ et on pose $v_{\gamma}=\mu\left(I_{\gamma}(\mathbb{Q}) \backslash I_{\gamma}(\mathbb{A})\right)>0$ et

$$
\mathrm{TO}_{\gamma}(f):=\int_{I_{\gamma}(\mathbb{A}) \backslash G(\mathbb{A})} f\left(g^{-1} \gamma \theta(g)\right) d i_{\mathbb{A}} \backslash d g_{\mathbb{A}} .
$$

On considérera aussi les version locales évidentes de ces intégrales orbitales tordues. 
Proposition 3.16. Pour toute fonction $f$ comme plus haut,

$$
\operatorname{trace}\left(R(f) I_{\theta}\right)=\sum_{\gamma \in\{G(\mathbb{Q})\}_{\mathrm{ell}}} v_{\gamma} \mathrm{TO}_{\gamma}(f)
$$

La somme porte sur un sous-ensemble fini de classes qui ne dépend que d'un compact de $G(\mathbb{A})$ contenant le support de $f$.

Preuve. Nous allons appliquer les résultats d'Arthur [A2] à la composante connexe G0. Ainsi que l'explique Arthur ([A1, p. 330], [A2, p. 528]), la validité de ces résultats dans ce cadre dépend de la vérification d'un argument de cohomologie galoisienne et de la validité du théorème de Paley-Wiener pour $G(\mathbb{R}) \theta$. Le premier a en fait été vérifié depuis en toute généralité par Kottwitz et Rogawski [KR], ainsi que le second dans notre cadre par Mezo $\underline{\mathrm{M}}$.

Les propriétés de pseudo-coefficients des fonctions $f_{\infty}$ et $f_{\ell}$ (2.7, Rem. 3.7) impliquent que la fonction $f$ est cuspidale au sens d'Arthur [A2, $\$ 7$ p. 538] en les deux places $\infty$ et $\ell$. Mieux, en la place $\ell$, les intégrales orbitales de $f_{\ell}$ en les éléments non semisimples $\mathbb{Q}_{l}$-elliptiques s'annulent par le Corollaire 3.14, de sorte que A2, Cor. 7.4] s'applique. Ce corollaire identifie le terme de droite de l'énoncé à la trace de $R(f) I_{\theta}$ dans la représentation de $G^{+}(\mathbb{A})$ sur

$$
L_{\text {disc }}^{2}\left(A_{G} \mathrm{GL}_{2 n}(\mathbb{Q}) \backslash \mathrm{GL}_{2 n}(\mathbb{A})\right) \text {. }
$$

Notons qu'Arthur considère loc. cit. une sommation précise pour cette trace partitionnée par les normes $t$ possibles des caractères infinitésimaux des $\Pi_{\infty}$. Comme $f_{\infty}$ ne trace que dans des représentations ayant même caractère infinitésimal, un seul de ces $t$ intervient. Il ne reste qu'à voir que si une $G^{+}(\mathbb{A})$-représentation automorphe irréductible discrète $\Pi$ n'est pas cuspidale, alors trace $\left(\Pi(f) I_{\theta}\right)=0$. Si cette trace est non nulle, alors $\Pi_{p}$ est de la forme $I(\chi)$ par le Théorème 3.3 (i). Mais le théorème de Moeglin-Walspurger exclut22 la classe inertielle de $(M, \omega \times \check{\omega})_{G}$ comme composante locale possible d'une représentation résiduelle de $\mathrm{GL}_{2 n}$ car $\omega$ n'est isomorphe à aucune torsion non ramifiée de $\check{\omega}$.

3.16.1. Preuve du théorème. Rappelons que la fonction $f_{\infty}$ dépend notamment du choix d'une série $\theta$-discrète cohomologique de $G(\mathbb{R})$, qui sont indexées comme on l'a vu au $\$ 2.1$ par les représentations irréductibles du groupe compact $\mathrm{SO}_{2 n+1}(\mathbb{R})$. Pour fixer les idées, on choisit $T$ un tore maximal de ce dernier et on note $V_{\lambda}$ la représentation irréductible de poids extrémal $\lambda \in X^{*}(T)$. Pour chaque tel $\lambda$, on fixe un pseudo-coefficient $f_{\infty}=f_{\lambda}$ de la série $\theta$-discrète $\pi_{\lambda}$ associée de sorte que le support de tous ces $f_{\infty}, \lambda$ variant, soit contenu dans un même compact de $G(\mathbb{R})$, ce qui est loisible, et on applique la Proposition 3.16 aux fonctions

$$
f^{\lambda}:=f_{\lambda} \otimes f_{\ell} \otimes f_{p} \otimes f^{\infty, \ell, p} .
$$

Supposons que la trace de $f^{\lambda}$ dans une représentation cuspidale $\rho$ est non nulle. Alors $\rho_{\infty}$ est générique, a le même caractère infinitésimal que $\pi_{\lambda}$, et donc lui est isomorphe. D'après les propriétés de pseudo-coefficients de $f_{\ell}$ (Prop. 3.8 (i)', noter que la triviale n'est jamais composante locale d'une cuspidale de $G$ ) et $f_{p}$ (Théorème 3.3 (i)), il suffit donc de montrer que l'on peut choisir $\lambda$ de sorte que trace $\left(R\left(f^{\lambda}\right) I_{\theta}\right) \neq 0$,

\footnotetext{
${ }^{22}$ On aurait aussi pu arguer en $\ell$, ou même en l'infini en supposant de plus que le caractère infinitésimal de la série $\theta$-discrète attachée à $f_{\infty}$ est suffisamment régulier.
} 
soit encore que le terme géométrique correspondant de la formule de trace de la Proposition 3.16 est non nul.

La somme du côté géométrique est à support inclus dans un ensemble fini

$$
\Sigma \subset\{G(\mathbb{Q})\}_{\text {ell }}
$$

indépendant de $\lambda$. On va voir qu'asymptotiquement en $\lambda$ la classe de $\gamma_{0}$ porte le terme principal elliptique de la formule des traces. Les propriétés essentielles de $\gamma_{0}$ sont résumées dans le lemme suivant.

Lemme 3.17. L'élément $\gamma_{0}$ est à $\theta$-conjugaison près l'unique élément $\theta$-semisimple elliptique de $G(\mathbb{Q})$ tel que $\gamma_{0} \theta\left(\gamma_{0}\right)=-1$. Son centralisateur tordu est le sous-groupe symplectique de $G$ de matrice $\gamma_{0} J$. Sa classe de $\theta$-conjugaison stable coïncide avec sa classe de $\theta$-conjugaison.

Preuve. On a $\gamma_{0} \theta\left(\gamma_{0}\right)=-1$ et le centralisateur tordu de $\gamma_{0}$ est le groupe $\{g \in$ $\left.\mathrm{GL}_{2 n}, g \gamma_{0} J^{t} g=\gamma_{0} J\right\}$ i.e. le groupe symplectique usuel $\mathrm{Sp}_{2 n}$ de matrice $\gamma_{0} J: \gamma_{0}$ est donc bien $\theta$-semisimple elliptique.

Les matrices antisymétriques inversibles étant toutes congrues à $\gamma_{0} J$ dans $\mathrm{GL}_{2 n}(\mathbb{Q})$, la classe de $\theta$-conjugaison de $\gamma_{0}$ coïncide exactement avec l'ensemble des éléments $\gamma$ tels que $\gamma \theta(\gamma)=-1$. Pour la même raison, la classe de $\theta$-conjugaison stable de $\gamma_{0}$ est réduite à sa classe de $\theta$-conjugaison (ou directement, $H^{1}\left(\mathbb{Q}, \mathrm{Sp}_{2 n}\right)=$ $0)$.

Lemme 3.18. $\mathrm{TO}_{\gamma_{0}}\left(f^{\lambda, \infty}\right)$ est une constante non nulle.

Preuve. En effet, c'est le produit

$$
\mathrm{TO}_{\gamma_{0}}\left(f_{\ell}\right) \cdot \mathrm{TO}_{\gamma_{0}}\left(f_{p}\right) \cdot \mathrm{TO}_{\gamma_{0}}\left(f^{\infty, \ell, p}\right)
$$

Le terme $\mathrm{TO}_{\gamma_{0}}\left(f_{\ell}\right)$ (resp. $\mathrm{TO}_{\gamma_{0}}\left(f_{p}\right)$ ) est non nul d'après la Proposition 3.8 (ii) car $\gamma_{0}$ est $\theta$-semisimple elliptique (resp. d'après le Théorème 3.3 (ii)). Le terme $\mathrm{TO}_{\gamma_{0}}\left(f^{\infty, \ell, p}\right)$ est un réel strictement positif car $\gamma_{0} \in \prod_{v \neq \infty, \ell, p} G\left(\mathbb{Z}_{v}\right)$.

Si $\gamma \in G(\mathbb{Q})$ est un élément $\theta$-semisimple, voyons-le comme un élément $\theta$ semisimple de $G(\mathbb{R})$ et considérons sa norme $\mathcal{N} \gamma \in \mathrm{SO}_{2 n+1}(\mathbb{R})$ définie à la fin du 2.7. Par définition, $\mathcal{N} \gamma$ est un élément dont la classe de conjugaison sous $\mathrm{SO}_{2 n+1}(\mathbb{R})$ ne dépend que de la classe de $\theta$-conjugaison de $\gamma$ sous $G(\mathbb{R})$. D'après le Théorème 2.12, on sait que pour une normalisation convenable des mesures on a pour tout $\lambda$,

$$
\mathrm{TO}_{\gamma}\left(f_{\lambda}\right)= \pm \operatorname{trace}\left(\mathcal{N} \gamma, V_{\lambda}\right)
$$

Notons que la classe de $\gamma_{0}$ est l'unique classe de norme centrale (i.e., triviale) dans $\mathrm{SO}_{2 n+1}(\mathbb{R})$. En effet, par définition, $\mathcal{N} \gamma=1$ si et seulement si $\gamma \theta(\gamma)=-1$, et on conclut par le Lemme 3.17. De plus,

$$
\left|\mathrm{TO}_{\gamma_{0}}\left(f^{\lambda}\right)\right|=c \cdot \operatorname{dim}\left(V_{\lambda}\right) \neq 0
$$

pour une certaine constante $c>0$ d'après (3.7) et le Lemme 3.18, Le Corollaire 1.12 montre alors que lorsque $\lambda$ tends vers l'infini dans $X^{*}(T) \otimes \mathbb{R}$ en s'éloignant des murs,

$$
\frac{\mathrm{TO}_{\gamma}\left(f^{\lambda}\right)}{\operatorname{dim}\left(V_{\lambda}\right)} \longrightarrow 0, \quad \forall \gamma \in \Sigma \backslash\left\{\gamma_{0}\right\}
$$


puis que

ce qui conclut.

$$
\left|\operatorname{trace}\left(R\left(f^{\lambda}\right) I_{\theta}\right)\right| \sim v_{\gamma_{0}} \cdot c \cdot \operatorname{dim}\left(V_{\lambda}\right),
$$

Remarque 3.19. On démontrera au $\$ 4.17$ qu'en normalisant correctement les $f^{\lambda}$ le signe \pm intervenant dans la formule (3.7) est en fait +1 si $\gamma=\gamma_{0}$ (indépendamment de $\lambda$ ). L'argument ci-dessus prouvera alors la formule (0.2) annoncée dans l'introduction.

\section{Non-Évanouissement D'Une intéGrale orbitale tordue}

Le but de ce chapitre est de démontrer les propriétés de la fonction $f_{p}$ utilisée dans le Chapitre 3. Bien que le problème soit local, la démonstration utilise la formule des traces tordue pour $\mathrm{GL}(2 n)$. Elle s'est révélée assez difficile; en revanche sa portée est assez grande : nous esquissons dans le 4.18 les conséquences de la méthode. Celle-ci suggère des propriétés intéressantes de la formule de Plancherel tordue (associée à $\mathrm{GL}(2 n) / \mathbb{Q}_{p}$ et à l'automorphisme $\theta$ ), ainsi que des intégrales orbitales tordues de coefficients de supercuspidales $\theta$-stables selon la parité (i.e., la nature symplectique ou orthogonale) de la représentation galoisienne associée.

La preuve de la non-nullité de $\mathrm{TO}_{\gamma_{0}}\left(f_{p}\right)$ en l'élément « principal » $\gamma_{0}$ du Chapitre 3 repose sur le modèle de Whittaker et un argument simple mais nouveau de positivité (4.13). Il nous a imposé de préciser le Théorème 2.12, en éliminant le signe implicite dans celui-ci grâce à la normalisation « de Whittaker » pour l'entrelacement $A$ entre $\pi$ et $\pi \circ \theta$.

Ceci nous impose hélas de remplacer l'automorphisme $\theta$ « de Waldspurger » (2.1) par l'automorphisme $\theta_{0}$ respectant le modèle de Whittaker. Les traductions nécessaires (pour aboutir au résultat utilisé dans le Chapitre 3) sont faites dans le 4.7

4.1. Pseudo-coefficients. Rappelons qu'on a fixé au 3.1 une représentation supercuspidale $\omega$ de $\operatorname{GL}\left(n, \mathbb{Q}_{p}\right)$. Pour simplifier nous supposons $\omega$ non autoduale, même modulo twist non-ramifié :

$$
\widetilde{\omega} ¥ \omega \otimes \chi, \quad \forall \chi \in X_{\mathrm{nr}}\left(\mathbb{Q}_{p}^{\times}\right)
$$

où $X_{\text {nr }}\left(\mathbb{Q}_{p}^{\times}\right) \cong \mathbb{C}^{\times}$est le tore des caractères non-ramifiés. (Le lecteur se convaincra aisément que cette hypothèse n'est pas fondamentale.) On écrira simplement $\omega \chi$ pour $\omega \otimes \chi$.

Rappelons que $\theta(g)=J_{2 n}{ }^{t} g^{-1} J_{2 n}$, où nous notons maintenant $J_{2 n}$ la matrice $J$ du Chapitre 2. De même,

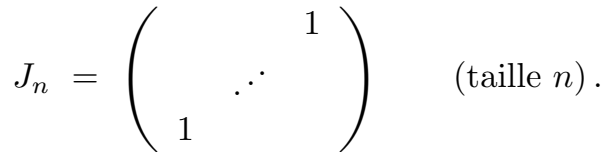

On désignera aussi par $\theta$ l'automorphisme $g \longmapsto J_{n}{ }^{t} g^{-1} J_{n}$ de GL(n).

Nous nous intéressons à la représentation induite

$$
I(\chi)=\operatorname{ind}_{P}^{G}\left(\omega \chi \otimes \widetilde{\omega} \chi^{-1}\right)
$$

de $\mathrm{G}=\mathrm{GL}\left(2 n, \mathbb{Q}_{p}\right)$. L'induction est unitaire; $P$ est le parabolique (triangulaire supérieur) de type $(n, n) ; \chi \in X_{\mathrm{nr}}$. Vu notre hypothèse sur $\omega, I(\chi)$ est irréductible 
pour tout $\chi$; elle est unitaire si, et seulement si, $\chi$ est unitaire. (En effet si $I(\chi)$ est unitaire, donc hermitienne, on doit avoir

$$
\overline{I(\chi)}=\operatorname{ind}\left(\overline{\omega \chi} \otimes \overline{\widetilde{\omega}} \bar{\chi}^{-1}\right) \cong \operatorname{ind}\left(\widetilde{\omega} \chi^{-1} \otimes \omega \chi\right)=\widetilde{I(\chi)} .
$$

Or $\bar{\omega} \cong \widetilde{\omega} ;$ vu notre hypothèse sur $\omega$, ceci implique $\omega \bar{\chi}^{-1} \cong \omega \chi$ soit $\omega \cong \omega(\chi \bar{\chi})$; alors $\chi \bar{\chi}$, donc $\chi$, est unitaire.)

Il est clair que $I(\chi)$ est isomorphe à sa duale et donc à $I(\chi)^{\theta}$. Pour obtenir un entrelacement explicite, remarquons que $\widetilde{\omega} \chi^{-1}=\widetilde{\omega \chi}$ est isomorphe à $(\omega \chi) \circ \theta=$ $(\omega \chi)^{\theta}$. Nous réalisons donc $I(\chi)$ comme l'induite, isomorphe à (4.1) et désignée par la même notation :

$$
I(\chi)=\operatorname{ind}_{P}^{G}\left(\omega \chi \otimes(\omega \chi)^{\theta}\right) .
$$

Si $V$ est l'espace de $\omega$, l'espace $\mathcal{L}(\chi)$ de $I(\chi)$ est donc formé des fonctions $f$ : $G \longrightarrow V \otimes V$ vérifiant, pour $p=\operatorname{diag}\left(m_{1}, m_{2}\right) n \in P=(\operatorname{GL}(n) \times \operatorname{GL}(n)) N$ :

$$
f(p g)=\delta_{P}(p)^{1 / 2}\left((\omega \chi)\left(m_{1}\right) \otimes(\omega \chi)^{\theta} m_{2}\right) f(g) .
$$

Soit $A_{M}: V \otimes V \longrightarrow V \otimes V$ l'opérateur

$$
v \otimes w \longmapsto w \otimes v,
$$

entrelaçant $\omega \chi \otimes(\omega \chi)^{\theta}$ et $(\omega \chi)^{\theta} \otimes \omega \chi$. On définit

$$
A_{\theta}: \mathcal{L}(\chi) \longrightarrow \mathcal{L}(\chi)
$$

par

$$
A_{\theta} f(g)=A_{M} f(\theta g) .
$$

On vérifie aussitôt que $A_{\theta}$ préserve $\mathcal{L}(\chi)$; il entrelace évidemment les représentations $I(\chi)$ et $I(\chi) \circ \theta$; il est involutif. Noter que, dans la réalisation compacte de $I(\chi)$, $A_{\theta}$ est indépendant de $\chi$.

Proposition 4.2. Il existe une fonction $f=f_{p}$ sur $\mathrm{G}=\mathrm{G}\left(\mathbb{Q}_{p}\right)$ ayant les propriétés suivantes:

(i) $f \in \mathcal{C}_{c}^{\infty}(\mathrm{G})$,

(ii) trace $\left(A_{\theta} I(\chi)(f)\right)$ est une fonction algébrique sur $X_{n r},>0$ sur les caractères unitaires,

(iii) si $\pi$ est une représentation $\theta$-stable de $\mathrm{G}$ et $A: \pi \cong \pi \circ \theta$ est un opérateur d'entrelacement,

$$
\operatorname{trace}(A \pi(f)))=0
$$

si $\pi$ n'est pas une induite $I(\chi)$.

On appellera parfois une telle fonction $f$ un pseudo-coefficient positif. Nous donnons une démonstration simple de ce résultat; une autre démonstration utiliserait le théorème de Paley-Wiener tordu de Rogawski [Ro] ; cf. \$4.13.

Preuve. Le groupe $X_{n r}$ des caractères non-ramifiés de $\mathbb{Q}_{p}^{\times}$est un tore complexe; soit $\Gamma \subset X_{n r}$ le sous-groupe (fini) des caractères $\chi$ tels que $\omega \chi \cong \chi$ et soit $T=X_{n r} / \Gamma \cong$ $\mathbb{C}^{\times}$. L'orbite de Bernstein $\mathrm{Be}$ contenant les représentations $I(\chi)$ s'identifie à $T \times T$, par

$$
\left(\chi_{1}, \chi_{2}\right) \longmapsto \operatorname{ind}_{P}^{G}\left(\omega \chi_{1} \otimes \widetilde{\omega} \widetilde{\chi}_{2}\right)=I\left(\chi_{1}, \chi_{2}\right),
$$

et l'ensemble des $I(\chi)$ au sous-tore diagonal. Parmi les $I\left(\chi_{1}, \chi_{2}\right)$ seules les $I(\chi)$ sont autoduales. D'après des arguments bien connus, il existe $f \in C_{c}^{\infty}(G)$ telle que $\operatorname{trace}\left(A_{\theta} I(1)(f)\right) \neq 0$. D'après Bernstein, on peut alors remplacer $f$ par une fonction 
vérifiant, de surcroît, (iii). La fonction $F(\chi)=\operatorname{trace}\left(A_{\theta} I(\chi)(f)\right)$ est algébrique, non nulle, sur $T$; vu les propriétés de $\omega$, l'image du centre de Bernstein $\mathcal{Z}$ dans $\mathbb{C}[T \times T]$ est formée de toutes les fonctions algébriques. Soit $z$ le paramètre sur $T \cong \mathbb{C}^{\times}$, et

$$
F(\chi)=F(z)=\sum a_{n} z^{n}
$$

une série de Laurent finie. Si $h \in \mathcal{Z}$ est d'image

$$
H(\chi)=H(z)=\sum \bar{a}_{n} z^{-n}
$$

et si $g=h \star f$, on a alors :

$$
\operatorname{trace}\left(A_{\theta} I(\chi)(g)\right)=H(z) F(z),
$$

fonction positive sur les caractères unitaires, d'où (ii), la positivité stricte résultant aussitôt d'un argument de compacité (prendre une somme de telles fonctions).

Rappelons (Lemme 3.17) que l'élément

$$
\gamma_{0}=\left(\begin{array}{cc}
1_{n} & \\
& -1_{n}
\end{array}\right) \in G
$$

donne le «terme principal» de la formule des traces tordue (\$3.16.1). On a $\mathcal{N} \gamma_{0}=$ $1 \in \mathrm{SO}(2 n+1)$. Si $f \in \mathcal{C}_{c}^{\infty}\left(G\left(\mathbb{Q}_{p}\right)\right)$, l'intégrale orbitale tordue de $f$ en $\gamma_{0}$ est

$$
\mathrm{TO}_{\gamma_{0}}(f)=\int_{\mathrm{G}\left(\mathbb{Q}_{p}\right) / I\left(\mathbb{Q}_{p}\right)} f\left(g \gamma_{0} g^{-\theta}\right) \frac{d g}{d i} .
$$

Elle est stable (Lemme 3.17).

Théorème 4.3. Si $f$ est un pseudo-coefficient positif (Prop. 4.2), $\mathrm{TO}_{\gamma_{0}}(f) \neq 0$.

4.4. Stabilité d'un caractère tordu. Considérons la distribution sur $G=G\left(\mathbb{Q}_{p}\right)$ :

$$
f \longmapsto \operatorname{trace}\left(A_{\theta} I(\chi)(f)\right) \text {. }
$$

Comme dans le Chapitre 2, 2.2 , on dispose sur $\mathrm{G}\left(\mathbb{Q}_{p}\right)$ de la notion d'éléments $\theta$-semisimples, $\theta$-réguliers : c'est le cadre original de Waldspurger [W1]. D'après des résultats généraux [Clo1, Thm. 1] on sait que la distribution (4.3), que l'on notera $\Theta_{\chi, \theta}$, est une fonction localement intégrable sur $\mathrm{G}, \mathcal{C}^{\infty}$ sur les élément $\theta$-réguliers, invariante par $\theta$-conjugaison.

Proposition 4.5. Le caractère tordu $\Theta_{\chi, \theta}$ est invariant par $\theta$-conjugaison stable (sur les éléments fortement $\theta$-réguliers).

Preuve. En effet, toute formule pour le caractère tordu de la représentation induite (par exemple, le théorème d'Atiyah-Bott [Clo1, Prop. 6]) montre que le support de $\Theta_{\chi, \theta}$ est contenu dans l'ensemble des éléments de $\mathrm{G}$ qui sont $\theta$-conjugués à un élément de $M$. Si $\gamma \in \mathrm{G}$ est un élément fortement $\theta$-régulier, son centralisateur tordu $I$ est un tore (\$2.2), de dimension $n$. L'ensemble des classes de $\theta$-conjugaison dans la classe de conjugaison stable de $\gamma$ s'identifie à

$$
H^{1}\left(\mathbb{Q}_{p}, I\right)=\operatorname{Ker}\left[H^{1}\left(\mathbb{Q}_{p}, I\right) \longrightarrow H^{1}\left(\mathbb{Q}_{p}, \mathrm{G}\right)\right]
$$

Or le centralisateur tordu dans $M$ d'un élément fortement $\theta$-régulier $g$ de $M$ s'identifie à un tore maximal de $\mathrm{GL}(n)$. En effet, si $\theta$ désigne l'automorphisme $m \longmapsto$ $J_{n}{ }^{t} m^{-1} J_{n}$ de GL( $\left.n\right)$, l'automorphisme $\theta$ de GL(2n) restreint à $M$ est $\left(m_{1}, m_{2}\right) \longmapsto$ $\left(\theta m_{2}, \theta m_{1}\right)$. Par l'isomorphisme $\left(m_{1}, m_{2}\right) \longmapsto\left(m_{1},{ }^{\theta} m_{2}\right)$ il est conjugué à

$$
\left(m_{1}, m_{2}\right) \longmapsto\left(m_{2}, m_{1}\right) \text {. }
$$


On est alors ramené au cas, facile, de la $\sigma$-conjugaison pour le changement de base en une place décomposée [AC, Ch. I.5]. Au vu des dimensions, on en déduit que les centralisateurs tordus $I$ de $g$ dans $M$ et $\mathrm{G}$ coïncident. Donc $I$ est cohomologiquement trivial, d'où la proposition.

Corollaire 4.6. Si $f \in \mathcal{C}_{c}^{\infty}(G)$ est un pseudo-coefficient positif, les intégrales orbitales tordues stables de $f$, en les éléments fortement $\theta$-réguliers, ne sont pas identiquement nulles.

Preuve. Il suffit d'appliquer la formule d'intégration de Weyl sur $\theta \mathrm{G}$ à la fonction $f \cdot \Theta_{\chi, \theta}$.

4.7. De Waldspurger à Whittaker. Nous avons jusqu'ici utilisé l'automorphisme $\theta$ de $\mathrm{G}$ en suivant Waldspurger; mais les arguments qui suivent vont reposer sur le modèle de Whittaker, auquel il n'est pas adapté. Soit donc

$$
\begin{aligned}
& D=\operatorname{diag}(1,-1,1, \ldots, 1,-1),
\end{aligned}
$$

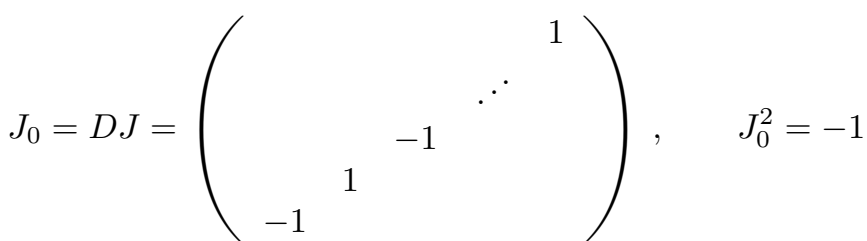

et $\theta_{0}: g \longmapsto J_{0}{ }^{t} g^{-1} J_{0}^{-1}(g \in \mathrm{GL}(2 n))$.

Adaptons rapidement les résultats précédents, en remplaçant $\theta$ par $\theta_{0} 23$ Rappelons tout d'abord que $\theta_{0}$ fixe un épinglage pour le couple $(B, T)$ formé du groupe de Borel triangulaire supérieur et du tore diagonal. Si $\alpha$ est un caractère additif non-trivial (d'un corps local $F=\mathbb{R}$ ou $\mathbb{Q}_{p}$, ou bien de $\mathbb{A}$ ) et si $\psi$ est le caractère du sous-groupe unipotent $N$ de $B$ donné par

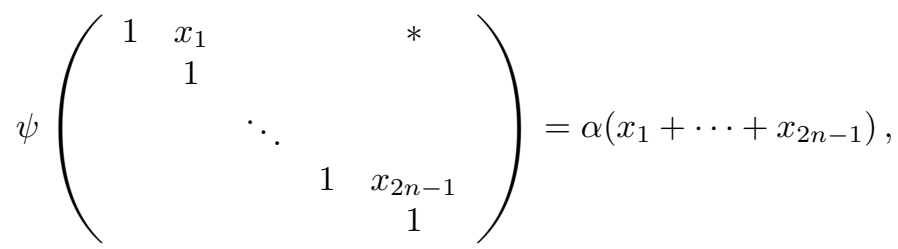

on a $\psi\left(\theta_{0} n\right)=\psi(n)(n \in N)$. Soit $\pi$ une représentation irréductible générique autoduale (de $G(F)$ ou $G(\mathbb{A})$ ). Il existe alors un unique opérateur d'entrelacement involutif $A_{\theta_{0}}^{\mathrm{W}}$ entre $\pi$ et $\pi \circ \theta$ tel que $A_{\theta_{0}}^{\mathrm{W}}(\lambda)=\lambda, \lambda$ étant l'unique fonctionnelle de Whittaker associée à $\psi$ (unique à un scalaire près) et $A_{\theta_{0}}$ opérant par l'action duale. On dira que $A_{\theta_{0}}^{\mathrm{W}}$ est la normalisation de Whittaker pour l'entrelacement involutif (a priori défini modulo \pm 1 ).

Si on néglige le signe, on peut construire, en $p$, un opérateur d'entrelacement $A_{\theta_{0}}$ sur $\pi=I(\chi)$ en posant

$$
A_{\theta_{0}}=\pi(D) A_{\theta}
$$

\footnotetext{
${ }^{23}$ Le lecteur choqué par notre inconstance méthodologique refera la construction de la norme (Ch. 2) en suivant Kottwitz et Shelstad [KS].
} 
On a alors

$$
\begin{aligned}
A_{\theta_{0}}^{2} & =\pi\left(D J^{t} D^{-1} J\right) A_{\theta}^{2} \\
& =\pi\left(J_{0}^{2}\right) A_{\theta}^{2} \\
& =\pi(-1)=1
\end{aligned}
$$

puisque $\pi$ est de caractère central trivial. Par ailleurs, notons, pour $\delta \in \mathrm{G}, \mathrm{T}_{0} \mathrm{O}_{\delta}(f)$ l'intégrale orbitale tordue déduite de $\theta_{0}$. Si $h(g)=f(g D)$, on vérifie aussitôt que

$$
\mathrm{T}_{0} \mathrm{O}_{\delta}(h)=\mathrm{TO}_{\gamma}(f)
$$

pour

$$
\delta=\gamma D .
$$

Par ailleurs trace $\left(A_{\theta_{0}} \pi(h)\right)=\operatorname{trace}\left(A_{\theta} \pi(f)\right)$. La fonction $h$ vérifie donc les propriétés de la Proposition 4.2 relativement à $\theta_{0}$. Le Théorème 4.3 est équivalent à

$$
\mathrm{T}_{0} \mathrm{O}_{\delta_{0}}(h) \neq 0,
$$

où

$$
\delta_{0}=\gamma_{0} D .
$$

Des considérations analogues s'appliquent à la place réelle, en remplaçant la norme du Chapitre 2 par $\mathcal{N}_{0}(\delta)=\mathcal{N}(\delta D)$. En particulier $\delta_{0}$ est de norme 1, l'analogue du Théorème 2.12 est vérifié, et $\delta_{0}$ est, localement ou globalement, l'unique élément dans sa classe de conjugaison tordue stable. Si $\pi$ est une représentation cohomologique de $G(\mathbb{R})$ comme dans le Chapitre 2, on définit $A_{\theta_{0}}$ comme dans le cas $p$-adique; alors, en posant encore $h_{\pi}(g)=f_{\pi}(g D)$ :

$$
\mathrm{T}_{0} \mathrm{O}_{\delta_{0}}\left(h_{\pi}\right)=\varepsilon(\pi) \operatorname{dim}\left(\pi_{H}\right),
$$

où $\varepsilon$ est un signe. La démonstration va nous amener à préciser 4.5 ainsi que le Théorème 4.3 Pour simplifier les notations, $\mathrm{TO}, \mathcal{N} \ldots$ désignent dorénavant les variantes relatives à $\theta_{0}$.

Proposition 4.8. Si l'opérateur $A_{\theta_{0}}=A_{\theta_{0}}^{\mathrm{W}}$ est normalisé pour le modèle de Whittaker, alors

(i) $\mathrm{TO}_{\delta_{0}}\left(h_{p}\right)>0$,

(ii) $\mathrm{TO}_{\delta_{0}}\left(h_{\pi}\right)=\operatorname{dim}\left(\pi_{H}\right)$ pour toute représentation cohomologique $\pi$ de $G(\mathbb{R})$ (pour une normalisation fixe des mesures sur $G(\mathbb{R})$ et le centralisateur tordu de $\left.\delta_{0}\right)$.

Nous reportons au $\$ 4.13$ et $\$ 4.17$ la démonstration de cette proposition. Nous aurons enfin besoin du

Lemme 4.9. Soit $\pi$ une représentation autoduale de la série principale : $\pi=$ $\operatorname{ind}\left(\chi_{1}, \ldots \chi_{n}, \chi_{n}^{-1}, \ldots, \chi_{1}^{-1}\right)$, les $\chi_{i}$ étant non ramifiés. Alors l'opérateur $A_{\theta_{0}}^{\mathrm{W}}$ donné par $f(g) \longmapsto f\left(\theta_{0}(g)\right)$ entrelace $\pi$ et $\pi \circ \theta_{0}$; il est involutif et opère par +1 sur l'espace de Whittaker; il opère trivialement sur le vecteur non-ramifié.

C'est évident (rappelons que la fonctionnelle de Whittaker est donnée par

$$
f \longmapsto \int_{N} f\left(w_{0} n\right) \psi(n) d n
$$

si $f$ est à support dans $B w_{0} N, w_{0}$ étant un représentant dans $G(F)$, que l'on peut choisir invariant par $\theta_{0}$, de l'élément de plus grande longueur du groupe de Weyl). 
4.10. Formule des traces (stable). Soit $f$ une fonction $\mathcal{C}^{\infty}$ à support compact sur $G(\mathbb{A})$, décomposée, donc $f=\bigotimes_{v}^{\prime} f_{v}$. À la place réelle, on choisit une représentation cohomologique $\pi_{\infty}$ autoduale de $G(\mathbb{R})$; on notera parfois $\mu$ le paramètre

$$
\left(m_{1}, \ldots, m_{n}\right) \in \mathbb{N}^{n}, m_{1} \geq \cdots \geq m_{n} \geq 0
$$

de $\pi=: \pi_{\mu} ;$ cf. \$2.1. C'est donc le plus haut poids de la représentation de $\mathrm{SO}(2 n+1)$ associée à $\pi$. Soit $\theta_{\mu}$ le caractère de celle-ci. Alors $f_{\pi}=f_{\mu}$ est le pseudo-coefficient de $\pi$ pour la normalisation de Whittaker de l'entrelacement $A_{\theta_{0}}$ (fonction notée $h_{\pi}$ dans le paragraphe précédent).

En la place $\ell, f$ est un pseudo-coefficient tordu pour la représentation de Steinberg (3.4). En $p$, c'est un pseudo-coefficient positif pour $I(\chi)$ (4.1). On utilise de nouveau $A_{\theta_{0}}$, avec la normalisation de Whittaker. En $v \neq \infty, \ell, p, f_{v}$ est pour l'instant arbitraire.

Soit $I_{\theta_{0}}: \varphi(x) \longmapsto \varphi\left(\theta_{0} x\right)$ l'opérateur d'entrelacement de $L^{2}(A \mathrm{G}(\mathbb{Q}) \backslash \mathrm{G}(\mathbb{A}))$ donné par $\theta_{0}$. Grâce aux propriétés particulières des fonctions $f_{\infty}$ et $f_{\ell}$, on a tout d'abord :

$$
\sum_{\delta \in\{\mathrm{G}(\mathbb{Q})\}_{\mathrm{ell}}} \operatorname{vol}\left(I_{\delta}\right) \mathrm{TO}_{\delta}(f)=\sum_{\pi} \operatorname{trace}\left(I_{\theta_{0}} \pi(f)\right) .
$$

On a fixé une mesure de Haar $d g_{\mathbb{A}}$ sur $G(\mathbb{A})$, qui définit la trace dans le membre de droite. Dans le membre de gauche, $\{\mathrm{G}(\mathbb{Q})\}_{\text {ell }}$ est l'ensemble des classes de conjugaison tordue d'éléments $\theta$-elliptiques [A2, p. 508] de $\mathrm{G}(\mathbb{Q}) ; \mathrm{TO}_{\delta}$ est l'intégrale orbitale tordue (adélique) définie par $d g_{\mathbb{A}}$ et une mesure de Haar $d i_{\mathbb{A}}$ sur le centralisateur tordu connexe $I_{\delta} \operatorname{de} \delta ; \operatorname{vol}\left(I_{\delta}\right)$ est la mesure de $I_{\delta}(\mathbb{Q}) \backslash I_{\delta}(\mathbb{A})$. La somme est finie. Dans le membre de droite, $\pi$ parcourt les représentations cuspidales $\theta_{0}$-stables (三 autoduales) de $\mathrm{G}(\mathbb{A})$.

L'égalité (4.6) entre le côté géométrique et le côté spectral est démontrée par Arthur [A2, §7]. L'expression du côté géométrique est [A2, Cor. 7.4], étant données les propriétés de $f_{\infty}$ et $f_{\ell}$. De même, le côté spectral est [A2, Cor. 7.2]. Noter que le membre de droite de [A2, Cor. 7.2] est à priori plus compliqué : il contient les représentations du spectre résiduel, ainsi que certaines induites $\theta$-discrètes : ces représentations sont éliminées par $f_{p}$ (cf. Prop. 3.16). Il contient aussi une sommation sur $t$ (norme du caractère infinitésimal de $\pi_{\infty}$ ) mais $t$ est fixé par le choix de $f_{\infty}$. En particulier, $f_{\text {fin }}=\bigotimes_{v \neq \infty}^{\prime} f_{v}$ étant fixée, et donc aussi la ramification, le nombre de termes du membre de droite est fini.

Notons $\mathrm{T}(f)$ l'expression (4.6). Si $\delta \in G(\mathbb{Q})$ est un élément $\theta$-semisimple elliptique, $\mathrm{TO}_{\delta}(f)$ est un produit

$$
\prod_{v} \mathrm{TO}_{\delta}\left(f_{v}\right)
$$

d'intégrales orbitales locales. Si $\delta$ est $\theta$-semisimple et fortement régulier, posons

$$
\operatorname{STO}_{\delta}(f)=\prod_{v} \operatorname{STO}_{\delta}\left(f_{v}\right)
$$

où

$$
\operatorname{STO}_{\delta}\left(f_{v}\right)=\sum_{\delta^{\prime}} \operatorname{TO}_{\delta}\left(f_{v}\right)
$$

la somme portant sur les éléments $\delta^{\prime}$ stablement $\theta$ conjugués à $\delta$. Il résulte alors de la stabilisation du terme régulier de la formule des tracespar Kottwitz-Shelstad 
KS que :

Lemme 4.11. Soit $q$ un nombre premier différent de $p$, $\ell$ et soit $f=\bigotimes_{v}^{\prime} f_{v}$ une fonction vérifiant les conditions précédentes, $f_{q}$ étant de plus de support $\theta$-fortement régulier. Alors

$$
\mathrm{T}(f)=\alpha(\mathrm{G}) \sum_{\delta} \operatorname{STO}_{\delta}(f)
$$

la somme portant sur les mêmes éléments qu'en (4.6) mais modulo $\theta$-conjugaison stable dans $\mathrm{G}$.

Ceci résulte de [KS, Ch. 7] (cf. en particulier (7.4.3); $\alpha(\mathrm{G})$ est une constante $>0$ ) et des propriétés de $f_{\infty}$ : c'est une fonction stabilisante ([La1, §3.8] et notre Corollaire 2.13). On en déduit :

Proposition 4.12. Il existe une représentation cuspidale, $\theta$-stable $\pi$ de $\mathrm{G}(\mathbb{A})$ telle que :

(i) $\pi_{\infty} \simeq \pi_{\mu}$,

(ii) $\pi_{\ell} \simeq \mathrm{St}_{\ell}$,

(iii) $\pi_{p} \simeq I(\chi)$ pour un caractère non-ramifié $\chi$,

Preuve. Soit en effet $f_{q}$ la fonction caractéristique d'un ouvert compact $\omega_{q}$ de $\mathrm{G}\left(\mathbb{Q}_{q}\right)$ constitué d'éléments $\theta$-semisimples fortement réguliers. Alors

$$
\mathrm{T}(f)=\alpha(G) \sum_{\delta} \mathrm{STO}_{\delta}(f),
$$

la somme étant uniformément finie quand le support de $f$ est fixé [KS, p. 106]. L'application $\mathcal{N}_{0}$ donne une bijection entre classes de $\theta_{0}$-conjugaison semisimples $\delta$ stable et classes de conjugaison stable semisimples dans $\mathrm{SO}(2 n+1)^{*}$ (quasi-déployé) [KS] ; celles-ci sont données par les polynômes réciproques

$$
P(X) \in \mathbb{Q}[X], \quad P(X)=X^{2 n} P\left(X^{-1}\right)
$$

de degré $2 n$. On écrira $P=P_{\delta}$. En $v=\infty, \ell, p$, choisissons un élément $\theta$-semisimple fortement régulier $\delta_{v} \in \mathrm{GL}_{2 n}\left(\mathbb{Q}_{v}\right)$ de sorte que

$$
\mathrm{STO}_{\delta_{v}}\left(f_{v}\right) \neq 0
$$

ce qui est loisible d'après le Théorème 2.12, la Proposition 3.8 et le Corollaire 4.6. (Noter que le Corollaire 3.10 implique que les intégrales orbitales de $f_{\ell}$ sont non nulles et de signe constant sur les conjugués stables d'un élément $\theta$-elliptique fortement $\mathbb{Q}_{\ell}$-régulier.) Cette non-annulation persiste alors dans un voisinage ouvert compact assez petit $\omega_{\infty} \times \omega_{\ell} \times \omega_{p}$ de $\left(\delta_{\infty}, \delta_{\ell}, \delta_{p}\right)$ dans $\mathrm{GL}\left(2 n, \mathbb{A}_{\infty \ell}\right)$.

Par approximation faible on peut trouver $\delta^{\prime} \in \mathrm{GL}(2 n, \mathbb{Q})$ tel que (pour le plongement diagonal) $\delta^{\prime}$ appartient à $\prod_{v \in S} \omega_{v}$, où $S=\{\infty, \ell, p, q\}$. Soient $\omega^{S}=\prod_{v \notin S} \omega_{v}$ un voisinage ouvert compact décomposé fixe de $\delta^{\prime}$ dans $\mathrm{GL}_{2 n}\left(\mathbb{A}^{S}\right)$, et $f^{S}$ la fonction caractéristique de $\omega^{S}$. Si

$$
\operatorname{STO}_{\delta}\left(f_{S} \otimes f^{S}\right) \neq 0 \quad(\delta \in \mathrm{G}(\mathbb{Q}))
$$

le polynôme $P_{\delta}(X)$ a les propriétés suivantes. Ses coefficients sont des $S^{\prime}$-entiers où $S^{\prime}=S \cup\left\{v, \omega_{v} \neq \mathrm{GL}_{2 n}\left(\mathbb{Z}_{v}\right)\right\}$. En les places $v \in S^{\prime}$ ils sont bornés par la donnée de $\prod_{v \in S^{\prime}} \omega_{v}$ : il n'y a qu'un nombre fini de tels polynômes.

Choisissons $\omega_{q}$ contenant $\delta_{q}^{\prime}$ assez petit. $\operatorname{Si~}_{\mathrm{STO}_{\delta}}(f) \neq 0$, alors les coefficients de $P_{\delta}$ sont uniquement déterminés; donc $P_{\delta}=P_{\delta^{\prime}}: \delta$ est stablement conjugué à $\delta^{\prime}$. 
La somme (4.8) ne porte que sur la classe de conjugaison stable de $\delta^{\prime}$. Comme $\delta^{\prime}$ est fortement régulier, $\mathrm{STO}_{\delta^{\prime}}\left(f_{q} \otimes f^{S}\right)>0$, d'où la proposition.

4.13. Démonstration du Théorème 4.3. Dans ce paragraphe nous démontrons le théorème, en supposant pour l'instant la partie archimédienne (ii) de la Proposition 4.8. Noter que d'après le Chapitre 2, celle-ci est équivalente à

Proposition 4.14. Pour $\delta \in G(\mathbb{R})$ de norme elliptique,

$$
\mathrm{TO}_{\delta}\left(h_{\pi}\right)=\varepsilon(\delta) \operatorname{trace} \pi_{H}\left(\mathcal{N}_{0} \gamma\right)
$$

où le signe $\varepsilon(\delta)$ ne dépend pas de $\mu$; de plus $\varepsilon\left(\delta_{0}\right)=1$.

Comme dans le Chapitre 2, cette assertion n'est vraie que pour des choix convenables des mesures (positives) sur les centralisateurs tordus, choix que nous ne préciserons pas.

Nous utiliserons le résultat élémentaire suivant :

Proposition 4.15. Soit $G$ un groupe de Lie compact, $\gamma_{0}=1, \gamma_{1}, \ldots, \gamma_{N}$ des classes de conjugaison distinctes dans $G$, et $\lambda_{0}, \ldots, \lambda_{N}$ des nombres complexes. Supposons que, pour tout caractère irréductible $\rho$ de $G$,

$$
\sum_{i=0}^{N} \lambda_{i} \text { trace } \rho\left(\gamma_{i}\right)
$$

est un nombre réel $\geq 0$, et que cette somme soit strictement positive pour un caractère $\rho_{0}$. Alors $\lambda_{0}$ est un réel $>0$.

Preuve. Soit en effet $\mathcal{O}_{i}$ la classe de conjugaison de $\gamma_{i}$ et $\mu_{i}$ la mesure invariante (normalisée) sur $\mathcal{O}_{i}$, vue comme une distribution sur $G$. Si $\mu=\sum \lambda_{i} \mu_{i}$,

$$
\text { trace }(\rho(\mu)) \geq 0 \quad(\rho \text { irréductible }) \text {. }
$$

D'après l'extension à $\mathrm{G}$ de la transformation de Fourier des distributions, la distribution $\mu$ s'écrit

$$
\mu=\sum_{\rho} a_{\rho} \bar{\Theta}_{\rho}
$$

où $\Theta_{\rho}$ est le caractère de $\rho$ et $a_{\rho}$ est une fonction à croissance lente sur $\widehat{G}$. Il résulte des relations d'orthogonalité et de (4.9) que

$$
a_{\rho} \geq 0, \quad a_{\rho_{0}}>0 .
$$

Soit $f$ une fonction $\mathcal{C}^{\infty}$ sur $G$ telles que le support de $f * f^{*}$ ne rencontre pas $\mathcal{O}_{i}$ pour $i>0, f^{*}(g)$ étant $\overline{f\left(g^{-1}\right)}$. Alors

$$
\mu\left(f * f^{*}\right)=\lambda_{0}\|f\|^{2}=\sum_{\rho} a_{\rho} \text { trace } \rho\left(f * f^{*}\right),
$$

d'où le résultat. 
Soit $\pi$ la représentation exhibée dans la Proposition 4.12. Soit $q$ un nombre premier tel que $\pi_{q}$ est ramifiée. La représentation $\pi_{q}$ est générique, $\theta_{0}$-stable et même tempérée d'après Harris et Taylor $[\mathrm{HT}$. Elle s'écrit donc :

$$
\pi_{q}=\operatorname{ind}_{P}^{G} \quad\left(\delta_{1} \otimes \cdots \otimes \delta_{r}\right)
$$

où $P$ est un parabolique de type $\left(n_{1}, \ldots, n_{r}\right), \delta_{i}$ est unitaire et de carré intégrable pour GL $\left(n_{i}\right)$, et où l'on peut supposer :

(i) pour $i \leq t, n_{r+1-i}=n_{i}$ et $\delta_{i}=\delta_{r+1-i} \circ \theta_{0}$,

(ii) pour $t<i<r+1-t, \delta_{i} \cong \delta_{i} \circ \theta_{0}$.

(On a désigné par $\theta_{0}$ l'automorphisme du $\$ 4.7$ pour GL $\left(n_{i}\right)$, peut-être en remplaçant $D$ par $-D$.)

S'il n'y a que des blocs de type (i), on construit comme dans le 4.1 un entrelacement $A_{\theta_{0}}$, Whittaker-normalisé, pour $\pi=\pi_{q}$, ainsi que pour toutes les induites des $\delta_{i}$ tordues par des caractères non-ramifiés. En général, un tel $A_{\theta_{0}}$ existe : composer avec un opérateur d'entrelacement normalisé pour les blocs de type (ii); on obtient un opérateur opérant à priori par $\{ \pm 1\}$ sur la fonctionnelle de Whittaker. L'opérateur d'entrelacement normalisé étant holomorphe, le signe est constant.

Il résulte alors du théorème de Paley-Wiener de Rogawski [Ro] que :

Lemme 4.16. Il existe une fonction $f_{q} \in C_{c}^{\infty}\left(G\left(\mathbb{Q}_{q}\right)\right)$ telle que

(i) parmi les représentations $\theta$-stables génériques $\pi$ de $G\left(\mathbb{Q}_{q}\right)$, les $I\left(\delta_{i} \otimes \chi_{i}\right)$ (pour des $\chi_{i}$ non-ramifiés tels que l'induite est $\theta$-stable) sont les seules telles que

$$
\operatorname{trace}\left(A_{\theta_{0}} \pi\left(f_{q}\right)\right) \neq 0,
$$

(ii) trace $\left(A_{\theta_{0}}^{\mathrm{W}} I\left(\delta_{i} \otimes \chi_{i}\right)\left(f_{q}\right)\right)>0$

( $\chi_{i}$ unitaires non-ramifiés, I supposée $\theta$-stable).

Nous fixons ainsi $f_{q}$ pour tout nombre premier $q \neq \ell, p$ en lequel $\pi_{q}$ est ramifiée; $f_{\ell}$ et $f_{p}$ l'ont été, et nous faisons varier $f_{\infty}=f_{\mu}$ avec le poids $\mu$. Nous allons appliquer la formule des traces (4.6), $f_{\ell^{\prime}}$ (pour les autres nombres premiers) étant l'unité de l'algèbre de Hecke sphérique. Notons $S$ la réunion de $\{\infty\}$ et de l'ensemble des nombres premiers $q$ tels que $\pi_{q}$ est ramifiée.

Sur l'espace des formes paraboliques sur $G(\mathbb{A})$, on dispose d'une fonctionnelle de Whittaker globale, $\theta_{0}$-invariante :

$$
\varphi \longmapsto \int_{N(\mathbb{Q}) \backslash N(\mathbb{A})} \psi^{-1}(n) \varphi(n) d n=\lambda(\varphi) .
$$

Soit $\rho \subset L^{2}(A G(\mathbb{Q}) \backslash G(\mathbb{A}))$ une représentation cuspidale $\theta$-stable, où $A=\mathbb{R}_{+}^{\times} \subset$ $\mathrm{G}(\mathbb{R})$, et soit $A_{\theta_{0}}=\left.I_{\theta_{0}}\right|_{\rho}$. Le Lemme 4.9 implique que $A_{\theta_{0}}$ s'écrit

$$
\left(\bigotimes_{v \in S} A_{\theta_{0, v}}\right) \otimes A_{\theta_{0}}^{S},
$$

les $A_{\theta_{0, v}}$ sont normalisés et $A_{\theta_{0}}^{S}$ est le produit tensoriel (bien défini) des opérateurs non-ramifiés.

Nous utilisons la formule des traces (4.6). Le membre de droite s'écrit

$$
\sum_{\rho} \prod_{v \in S} \operatorname{trace}\left(A_{\theta_{0}} \rho_{v}\left(f_{v}\right)\right) \text {. }
$$


Pour chaque $\rho$ (cuspidale, $\theta$-stable), chaque terme du produit est $\geq 0$; la somme est en fait finie car $\rho_{\infty}$ est cohomologique et le niveau de $\rho$ est fixé. Le membre de gauche comporte un nombre fini, fixe de termes, même quand $\mu$ (et $f_{\mu}$ ) varie. Il s'écrit

$$
a\left(\delta_{0}\right) \operatorname{deg}\left(\theta_{\mu}\right)+\sum_{\delta \neq \delta_{0}} a(\delta) \theta_{\mu}(\mathcal{N} \delta)
$$

où

$$
a(\delta)=\varepsilon(\delta) \operatorname{vol}\left(I_{\delta}\right) \prod_{v \neq \infty} \mathrm{TO}_{\delta}\left(f_{v}\right)
$$

pour tout $\delta$, et $\varepsilon\left(\delta_{0}\right)=1$. Le seul élément de norme 1 dans $\mathrm{SO}(2 n+1)$ est $\delta_{0}$; on peut réécrire (4.10) en regroupant les $\delta \neq \delta_{0}$ selon la conjugaison (tordue) stable, de sorte que (4.10) est de la forme

$$
a\left(\delta_{0}\right) \operatorname{deg}\left(\theta_{\mu}\right)+\sum_{\gamma^{\prime}} a\left(\gamma^{\prime}\right) \theta_{\mu}\left(\gamma^{\prime}\right)
$$

et les $\gamma^{\prime} \in \mathrm{SO}(2 n+1)$ sont des classes de conjugaison distinctes.

On obtient alors une somme de valeurs de caractères de $\mathrm{SO}(2 n+1)(\mathbb{R})$ vérifiant les hypothèses de la Proposition 4.15, la positivité stricte résultant de la Proposition 4.12 Donc

$$
\prod_{v \neq \infty} \mathrm{TO}_{\delta_{0}}\left(f_{v}\right)>0
$$

d'où le Théorème 4.3 .

4.17. Démonstration de la Proposition 4.8. Considérons d'abord une extension quadratique réelle $F$ de $\mathbb{Q}$. Soit $\infty, \infty^{\prime}$ les deux places archimédiennes, et soit $f=\bigotimes_{v}^{\prime} f_{v} \in \mathcal{C}_{c}^{\infty}\left(\mathrm{G}\left(\mathbb{A}_{F}\right)\right)$ une fonction égale à la fonction unité sphérique aux places finies, alors que

$$
f_{\infty}=f_{\mu}, \quad f_{\infty^{\prime}}=f_{\mu^{\prime}},
$$

$\mu, \mu^{\prime}$ étant deux poids dominants pour $\mathrm{SO}(2 n+1)$. Faisons pour simplifier l'hypothèse suivante précisant le Théorème 2.12 :

(H) Si $\gamma \in \mathrm{GL}(2 n, \mathbb{R}) \theta$ n'est pas semisimple, alors $\mathrm{TO}_{\gamma}\left(f_{\mu}\right)=0$ pour tout poids dominant $\mu$ pour $\mathrm{SO}(2 n+1)(\mathbb{R})$.

La formule des traces simplifiée d'Arthur, ainsi que les considérations du \$4.10, s'appliquent alors. L'argument du $\$ 4.13$ donne, pour tous $\mu, \mu^{\prime}$ :

$$
a\left(\delta_{0}\right) \operatorname{deg}\left(\theta_{\mu}\right) \operatorname{deg}\left(\theta_{\mu^{\prime}}\right)+\sum_{\gamma_{1} \neq 1} a\left(\gamma_{1}, \mu, \mu^{\prime}\right) \theta_{\mu}(\gamma) \theta_{\mu^{\prime}}\left(\gamma^{\prime}\right) \geq 0
$$

où

$$
a\left(\delta_{0}\right)=\varepsilon(\mu) \varepsilon\left(\mu^{\prime}\right) \operatorname{vol}\left(I_{\delta_{0}}\right) \prod_{v \nmid \infty} \mathrm{TO}_{\delta_{0}}\left(f_{v}\right),
$$

et où $\gamma_{1}=\left(\gamma, \gamma^{\prime}\right)$ parcourt un ensemble fini de classes de conjugaison du groupe $\mathrm{SO}(2 n+1)(\mathbb{R})^{2}$, et $a\left(\gamma_{1}, \mu, \mu^{\prime}\right)=a\left(\gamma_{1}\right) \varepsilon(\mu) \varepsilon\left(\mu^{\prime}\right)$. Noter que si $\delta \neq \delta_{0}$ et si $\gamma_{1}=\left(\gamma, \gamma^{\prime}\right)$ est associé à $\delta, \gamma$ et $\gamma^{\prime}$ sont $\neq 1$, la conjugaison stable (globale) étant impliquée par la conjugaison stable en une place. Les signes $\varepsilon(\mu)$, pour l'instant inconnus, sont donnés par l'égalité

$$
A_{\theta_{0}}^{\mathrm{W}}=\varepsilon(\mu) A_{\theta_{0}}
$$


où les deux opérateurs sont des entrelacements involutifs pour $\pi(\mu)$, celui de gauche étant Whittaker-normalisé, et celui de droite donnant, comme dans le Chapitre 2, l'identité correcte (sans signe) pour l'intégrale orbitale en $\delta_{0}$.

Fixons $\mu^{\prime}$ et faisons tendre $\mu$ vers l'infini « loin des murs ». Le terme dominant de (4.11) est celui relatif à $\delta_{0}$. Les intégrales orbitales tordues, aux places finies, étant $>0$ en $\delta_{0}$, on voit que $\varepsilon(\mu) \varepsilon\left(\mu^{\prime}\right)$ est $>0$ pour $\mu$ "assez loin des murs». Variant maintenant $\mu^{\prime}$, on en déduit que $\varepsilon\left(\mu^{\prime}\right)$ est constant. Appliquant enfin cet argument à un corps $F$ cubique, on voit que $\varepsilon(\mu)=1$. Un argument analogue démontre le résultat en $p$, ainsi d'ailleurs qu'en $\ell$ (le pseudo-coefficient de $\mathrm{St}_{\ell}$ étant Whittaker-normalisé).

L'hypothèse d'annulation $(H)$ est vraisemblable, car les propriétés de $f_{\mu}$ sont similaires à celles de la fonction d'Euler-Poincaré (\$3.4). Pour s'en passer, introduisons une place finie de $\mathbb{Q}$ décomposée en $\left(\ell, \ell^{\prime}\right)$ dans $F$ (resp. en $\left(\ell, \ell^{\prime}, \ell^{\prime \prime}\right)$ dans le cas cubique). On prend $f_{\ell}$ et $f_{\ell^{\prime}}$ des fonctions d'Euler-Poincaré, adaptées à l'entrelacement Whittaker-normalisé de la représentation de Steinberg. Alors $\mathrm{TO}_{\delta_{0}}\left(f_{\ell}\right) \mathrm{TO}_{\delta_{0}}\left(f_{\ell^{\prime}}\right)>0$, la formule simplifiée d'Arthur s'applique, et le reste de l'argument est identique.

4.18. Facteurs locaux des représentations cuspidales autoduales. Les arguments de cet article révèlent des propriétés remarquables des représentations autoduales, de GL $(2 n)$, tant globales (représentations automorphes) que locales, qui peuvent être comprises du point de vue de la fonctorialité entre motifs et représentations automorphes, ainsi qu'à l'aide des résultats conjecturaux d'Arthur.

Soit $\pi$ une représentation cuspidale autoduale de $\operatorname{GL}(2 n, \mathbb{A})$ où $\mathbb{A}=\mathbb{A}_{\mathbb{Q}}$. Conjecturalement, Langlands lui associe une représentation complexe, de degré $2 n$ :

$$
r: \mathcal{L}_{\mathbb{Q}} \longrightarrow \operatorname{GL}(2 n, \mathbb{C}),
$$

(cf. $[\mathrm{Lg}$ ) qui devrait être irréductible. Si $\pi$, donc $r$, est autoduale, il existe sur l'espace de $r$ une forme bilinéaire non-dégénérée unique, donc orthogonale ou symplectique, invariante par $r$. On dira que $r$ est symplectique ou orthogonale.

Si $\pi_{\infty}$ est cohomologique, la représentation $r_{\infty}$ de $\mathrm{W}_{\mathbb{R}}$ obtenue par restriction est symplectique et non orthogonale; donc $r$ doit être symplectique. Noter que ceci n'est pas vrai si on suppose que $\widetilde{\pi} \cong \pi \otimes \varepsilon$ où $\varepsilon$ est un caractère, même d'Artin : les formes de poids $k$ impair sur GL(2) donnent des représentations $\pi$ (normalisées, comme chez Langlands, de façon à être tempérées) telles que le paramètre de $\pi_{\infty}$ est sur $\mathbb{C}^{\times}$:

$$
z \longmapsto\left(\begin{array}{cc}
(z / \bar{z})^{\frac{k-1}{2}} & \\
& (\bar{z} / z)^{\frac{k-1}{2}}
\end{array}\right)
$$

et la représentation associée de $\mathrm{W}_{\mathbb{R}}$ est orthogonale.

S'il existe un nombre premier $p$ tel que $\pi_{p}$ appartient à la série (essentiellement) discrète, le paramètre de Langlands

$$
r_{p}: \mathrm{WD}_{\mathbb{Q}_{p}} \longrightarrow \mathrm{GL}(2 n, \mathbb{C}),
$$

bien défini d'après Harris et Taylor, est indécomposable, et en fait irréductible si on le considère comme une représentation

$$
r_{p}^{\prime}: \mathrm{W}_{\mathbb{Q}_{p}} \times \mathrm{SU}(2) \longrightarrow \mathrm{GL}(2 n, \mathbb{C}) .
$$

De nouveau, si $r_{p}^{\prime}$ est symplectique (on dira que $\pi_{p}$ est symplectique), $r$ doit être symplectique. 
Remarque 4.19. En dimension 2, une représentation irréductible autoduale est symplectique si, et seulement si, son déterminant est trivial, ce qui permet de donner un sens non conjectural aux prédictions ci-dessus et de les prouver. Soit $\pi$ une cuspidale autoduale de $\operatorname{GL}\left(2, \mathbb{A}_{F}\right)$ qui est discrète en une place $v$, et soit $\omega$ le caractère central de $\pi$ : alors $\omega=1$ si, et seulement si, $\omega_{v}=1$. En effet, $\omega^{2}=1$; donc si $\omega \neq 1$, c'est le signe $\omega_{E / F}$ d'une extension quadratique $E$ de $F$, et on a alors

$$
\check{\pi} \simeq \pi \otimes \omega_{E / F} \circ \operatorname{det}
$$

par multiplicité 1 . La théorie du changement de base quadratique montre que $\pi$ est l'induite automorphe d'un caractère de Hecke $\chi$ de $\mathbb{A}_{E}^{*}$ (non isomorphe à son conjugué). On peut alors considerer de manière non conjecturale le $L$-paramètre de $\pi$, à savoir $L(\pi)=\operatorname{Ind}_{\mathrm{W}_{E}}^{\mathrm{W}_{F}} \chi$, qui est compatible à toutes les places avec $\pi$. Comme $\pi_{v}$ est discrète, $L(\pi)_{\mid \mathrm{W}_{F_{v}}}$ est irréductible, en particulier $v$ n'est pas décomposé dans $E$, i.e. $\omega_{v}=\omega_{E_{v} / F_{v}} \neq 1$.

On peut aussi comprendre ces phénomènes du point de vue des résultats annoncés par Arthur [A4, Ch. 30]. D'après ceux-ci, une représentation $\theta$-stable (disons, cuspidale) de $\mathrm{GL}(2 n, \mathbb{A})$ proviendra par fonctorialité d'une représentation automorphe d'un groupe endoscopique $H$ où $H$ est :

$$
\begin{array}{lll}
\text { (a) } & \mathrm{SO}^{*}(2 n+1), & \widehat{H}=\mathrm{Sp}(2 n, \mathbb{C}), \\
\text { (b) } & \mathrm{SO}^{*}(2 n, \chi), & \widehat{H}=\mathrm{SO}(2 n, \mathbb{C})
\end{array}
$$

où les groupes $\mathrm{SO}^{*}$ sont quasi-déployés (donc déployés en dimension impaire) et où le groupe $\mathrm{SO}^{*}(2 n, \chi)$ est spécifié par un caractère d'Artin $\chi$ d'ordre 2 décrit par Arthur A4 (et déterminé par $\pi$ ). Des arguments analogues montrent alors que sous les hypothèses le type (symplectique ou orthogonal) de $\pi$ sera déterminé par celui de $\pi_{p}$; les résultats d'Arthur devraient rendre ceci inconditionnel.

Les méthodes de cet article, combinées aux résultats d'Harris et Taylor associant des représentations galoisiennes à $\pi$ ([HT], complétés par [TY] si $\pi_{v}$ est une représentation de Steinberg généralisée), permettent toutefois d'obtenir des résultats partiels intéressants. La démonstration des deux théorèmes suivants ne sera pas tout à fait complète (voir la Remarque 4.23 (ii)) car nous devrons admettre l'hypothèse locale $(H)$ introduite au $\S 4.17$, qui est l'analogue réel du Corollaire 3.14, Nous espérons revenir ultérieurement sur la vérification de cette condition.

Théorème 4.20 (Supposant $(H)$ ). Soient $F$ un corps totalement réel et $\pi$ une représentation automorphe cuspidale de $\mathrm{GL}_{2 n}\left(\mathbb{A}_{F}\right)$. On suppose que $\pi$ est autoduale, essentiellement de carré intégrable en au moins une place finie $w$, et cohomologique à toutes les places archimédiennes. Alors:

(i) Pour toute place $v$ de $F, \pi_{v}$ est symplectique.

(ii) Si $V_{\ell}$ est une représentation $\ell$-adique de $\operatorname{Gal}(\bar{F} / F)$ associée à $\pi|\cdot|^{(2 n-1) / 2}$, avec $w$ premier à $\ell$, alors il existe un accouplement symplectique non dégénéré et Galois-équivariant

$$
V_{\ell} \otimes V_{\ell} \longrightarrow \overline{\mathbb{Q}}_{\ell}(2 n-1)
$$

Remarque 4.21. L'hypothèse « $w$ premier à $\ell$ » dans (ii) ne serait pas nécessaire s'il on disposait, pour les représentations galoisiennes d'Harris et Taylor, d'un analogue du théorème de Saito identifiant en $w \mid \ell$ la représentation de Weil-Deligne donnée par la théorie de Fontaine. 
Si $v$ est finie, " $\pi_{v}$ symplectique » signifie que son paramètre de Langlands sous sa forme $\mathrm{SU}(2)$ préserve une forme bilinéaire alternée non-dégénérée. Du point de vue de la représentation de Weil-Deligne $(r, N)$ associée, il est équivalent de demander que $r$ (la représentation de $\mathrm{W}_{F_{v}}$ ) préserve une forme bilinéaire alternée non-dégénérée, et que $N$ est dans l'algèbre de Lie du groupe symplectique associé.

Dans le contexte de l'énoncé, comme $\pi_{v}$ est tempérée ([HT]), elle s'écrira alors

$$
\pi_{v}=\operatorname{ind}_{P}^{G}\left(\delta_{1}, \delta_{1}^{\theta}, \ldots \delta_{r}, \delta_{r}^{\theta}, \delta_{2 r+1}, \ldots, \delta_{s}\right)
$$

où les $\delta_{i}$ sont des représentations de la série (essentiellement) discrète de $\operatorname{GL}\left(n_{i}, F_{v}\right)$, et les représentations $\delta_{2 r+1}, \ldots, \delta_{s}$ sont autoduales, distinctes et symplectiques.

Démontrons maintenant le Théorème 4.20. D'après le \$2.1 le (i) vaut par hypothèse si $v$ est archimédienne.

Fixons $v$ finie, choisissons un $\ell$ premier à $w$ et $v$, puis considérons une représentation galoisienne $\ell$-adique $V_{\ell}$ attaché 24 à $\pi||^{(2 n-1) / 2}$. D'après [HT] et TY], $V_{\ell}$ est irréductible, compatible avec $\pi$ (en toutes les places finies premières à $\ell$ ) selon la correspondance de Langlands Frobenius semi-simplifiée; en particulier, cela nous fournit un accouplement non-dégénéré Galois-équivariant comme dans l'énoncé (unique à un scalaire près, et dont il faut montrer qu'il est symplectique). Ainsi 25 il suffit de prouver (i) quand $v=w$ pour l'avoir pour tout $v$, et (i) implique (ii). De plus, si $\pi_{w}$ est la représentation de Steinberg, alors (i) et (ii) sont satisfaits. Il suffit donc de démontrer le théorème suivant (en fait, (i) $\Rightarrow$ (ii) $\Rightarrow$ (v) suffit).

Théorème 4.22 (Supposant $(H)$ ). Soient $\pi_{w}$ une représentation autoduale (essentiellement) discrète de $\mathrm{GL}_{2 n}\left(F_{w}\right)$ et $f_{w}$ un pseudo-coefficient tordu de $\pi_{w}$. Les conditions suivantes sont équivalentes :

(i) $\pi_{w}$ est composante locale d'une représentation automorphe cuspidale $\pi$ de $\mathrm{GL}_{2 n}\left(\mathbb{A}_{F}\right)$ qui est autoduale, et cohomologique à toutes les places archimédiennes,

(ii) $\mathrm{TO}_{\gamma_{0}}\left(f_{w}\right) \neq 0$,

(iii) les intégrales orbitales tordues stables de $f_{w}$ ne sont pas identiquement nulles,

(iv) les intégrales orbitales tordues stables fortement régulières de $f_{w}$ ne sont pas identiquement nulles,

(v) $\pi_{w}$ est composante locale d'une représentation automorphe cuspidale $\pi$ comme en (i) et qui de plus est la représentation de Steinberg à une autre place finie.

Si l'une de ces propriétés est satisfaite, alors $\pi_{w}$ est symplectique.

Preuve. L'implication (i) $\Rightarrow$ (ii) découle du même raisonnement de positivité que dans le 4.13 . Quitte à faire un changement de base quadratique réel décomposé en $w$, on peut supposer que $F \neq \mathbb{Q}$. Pour toutes les places finies $v \neq w$ de $F$, choisissons un pseudo-coefficient positif $f_{v}$ de $\pi_{v}$ comme dans le Lemme 4.16. Enfin, pour chaque place archimédienne $v$ de $F$ on prend pour $f_{v}$ un pseudo-coefficient de séries théta-discrètes cohomologiques quelconque comme au §2.7; il dépend d'un

\footnotetext{
${ }^{24} \mathrm{~L}$ 'existence et les propriétés de compatibilité de $V_{\ell}$ se déduisent de manière standard de $\mathrm{HT}$ ] par changement de base à tous les $E F, E$ étant un corps quadratique imaginaire tel que $E F$ est décomposé au dessus de $w$ (voir $[\mathrm{T}$ Thm. 3.6] pour le cas $F=\mathbb{Q}$ ).

${ }^{25}$ On pourra remarquer que si une représentation de Weil-Deligne préserve un accouplement symplectique, alors sa Frobenius simplification (qui associe à chaque élément du groupe de Weil sa partie semisimple dans sa décomposition de Jordan) préserve le même accouplement.
} 
$[F: \mathbb{Q}]$-uple $\lambda$ de poids dominants de $\mathrm{SO}_{2 n+1}(\mathbb{R})$. En appliquant la formule des traces tordue d'Arthur à la fonction $\prod_{v}^{\prime} f_{v}$, qui est simplifiée grâce à l'hypothèse $(\mathrm{H})$, et en faisant varier $\lambda$, l'argument du $\$ 4.13$ montre que

$$
\forall v, \quad \mathrm{TO}_{\delta_{0}}\left(f_{v}\right) \neq 0
$$

donc (ii).

L'implication (ii) $\Rightarrow$ (v) est par exemple conséquence de la méthode du \$3.16.1 si l'on choisit la fonction test égale à $f_{w}$ en la place $w$ : l'appel au Théorème 3.3 est remplacé par (4.13).

(ii) $\Rightarrow$ (iii) est évident. Rappelons que les intégrales orbitales stables sont définies en tous les éléments $\theta$-semisimples, et sont limites faibles de telles intégrales pour des éléments fortement $\theta$-réguliers; donc (iii) $\Leftrightarrow$ (iv).

À partir de (iv), les arguments de la Proposition 4.12 démontrent (v).

Enfin, d'après les considérations précédentes, le Théorème 4.20 s'ensuit, ainsi donc que la dernière propriété.

Remarque 4.23. (i) En fait, ces propriétés sont sans doute équivalentes à « $\pi_{w}$ est symplectique ». C'est connu si $\pi_{w}$ est supercuspidale par les résultats de Henniart [He2] et Shahidi [Sh2, Prop. 5.1] rappelés dans l'introduction. Nous reviendrons sur ce point dans un travail ultérieur.

(ii) L'hypothèse $(H)$ nous a servi uniquement à justifier que nous disposons d'une formule des traces simplifiée dans la preuve de (i) $\Rightarrow$ (ii). Ainsi, les équivalences (ii) $\Leftrightarrow\left(\right.$ iii) $\Leftrightarrow$ (iv) du Théorème 4.22 , et le fait qu'elles entrainent $\pi_{w}$ symplectique, sont inconditionnelles; de même, le Théorème 4.20 est valable inconditionnellement si $\pi$ est la représentation de Steinberg à une place finie.

Notons enfin le phénomène purement local qui devrait résulter de nos méthodes. Considérons, sur $G\left(\mathbb{Q}_{p}\right)$, l'intégrale orbitale tordue $\mathrm{TO}_{\delta_{0}}$ (on utilise l'automorphisme $\theta_{0}$ Whittaker-normalisé). D'après des principes généraux, elle devrait s'écrire

$$
\mathrm{TO}_{\delta_{0}}(f)=\int_{\widehat{\mathrm{G}}_{\text {temp }}^{\theta_{0}}} \operatorname{trace}\left(A_{\theta_{0}} \pi(f)\right) d \mu(\pi)
$$

où $\widehat{G}_{\text {temp }}^{\theta_{0}}$ est la variété des représentations tempérées, $\theta_{0}$-stables de $G$, et $d \mu(\pi)$ une distribution (une mesure ?). La distribution $\mathrm{TO}_{\delta_{0}}(f)$ est stable; d'après une extension simple du Théorème 4.22, les intégrales orbitales tordues stables de pseudocoefficients (au sens du Lemme 4.16) d'une famille d'induites non symplectiques (i.e., ne vérifiant pas la condition suivant (4.12) ) s'annulent. On dispose de plus de l'argument de positivité du 4.13. Par conséquent, on s'attend au résultat suivant, sans doute accessible :

Conjecture 4.24. (i) Dans la formule (4.14), l'intégrale ne porte que sur les représentations symplectiques.

(ii) La distribution $d \mu(\pi)$ est une mesure positive.

Terminons en remarquant que certaines de ces propriétés feront sans doute partie des démonstrations complètes par Arthur des résultats annoncés dans A4]. 


\section{Application au groupe de Galois absolu de $\mathbb{Q}$}

Replaçons-nous dans le contexte de l'introduction. Soient $S$ un ensemble fini de nombres premiers, $\mathbb{Q}_{S}$ une extension algébrique maximale de $\mathbb{Q}$ non ramifiée hors de $S$ (et de l'infini) et $\mathrm{G}_{S}=\operatorname{Gal}\left(\mathbb{Q}_{S} / \mathbb{Q}\right)$ son groupe de Galois. Supposons $S \neq \emptyset$ et fixons $p \in S$ un nombre premier.

Théorème 5.1. Si $|S| \geq 2$, les applications naturelles $\operatorname{Gal}\left(\overline{\mathbb{Q}}_{p} / \mathbb{Q}_{p}\right) \longrightarrow \mathrm{G}_{S}$ sont injectives.

Preuve. On peut supposer que $S=\{\ell, p\}$ où $\ell$ est un nombre premier différent de $p$. Fixons un plongement $\mathbb{Q}_{S} \longrightarrow \overline{\mathbb{Q}}_{p}$, et notons

$$
E:=\mathbb{Q}_{p} \cdot \mathbb{Q}_{S}
$$

le compositum de $\mathbb{Q}_{p}$ et $\mathbb{Q}_{S}$ dans $\overline{\mathbb{Q}}_{p}$ : il s'agit de démontrer que $E=\overline{\mathbb{Q}}_{p}$. Comme $\operatorname{Gal}\left(\overline{\mathbb{Q}}_{p} / \mathbb{Q}_{p}\right)$ n'admet pas de sous-groupe fermé normal non-trivial rencontrant trivialement le sous-groupe d'inertie par [Ch, Lemme 4 (i)], il suffit de démontrer que $\mathbb{Q}_{p}^{\text {nr }} \cdot E=\overline{\mathbb{Q}}_{p}$, soit encore que $\mathbb{Q}_{p}^{\text {ab }} \cdot E=\overline{\mathbb{Q}}_{p}$ puisqu'il est évident que $\mathbb{Q}_{p}\left(\mu_{p^{\infty}}\right) \subset E$.

D'après [Ch, Lemme 4 (iii)], la clôture algébrique séparable d'un corps $k$ est le compositum de ses sous-extensions galoisiennes finies $K / k$ dont le groupe de Galois admet une representation linéaire complexe à la fois injective et irréductible. Fixons donc une telle extension $K$ de $k:=\mathbb{Q}_{p}$, il s'agit de montrer que $K \subset E . \mathbb{Q}_{p}^{\mathrm{ab}}$.

$\mathrm{Vu}$ le choix de $K$, il existe une représentation injective irréductible $\rho: \operatorname{Gal}\left(K / \mathbb{Q}_{p}\right)$ $\rightarrow \mathrm{GL}_{n}(\mathbb{C})$ pour un certain entier $n \geq 1$, que l'on voit par inflation comme une représentation continue irréductible

$$
\psi: \mathrm{W}_{\mathbb{Q}_{p}} \longrightarrow \mathrm{GL}_{n}(\mathbb{C})
$$

du groupe de Weil $W_{\mathbb{Q}_{p}}$ de $\mathbb{Q}_{p}$. Quitte à tordre $\psi$ par un caractère lisse $\eta$ bien choisi de $\mathrm{W}_{\mathbb{Q}_{p}}$, nous pouvons supposer que la représentation duale $\psi^{*}$ n'est isomorphe à aucune torsion de $\psi$ par un caractère non-ramifié. En effet, si $I \subset \mathrm{W}_{\mathbb{Q}_{p}}$ est le sousgroupe d'inertie, $I \cap \operatorname{Ker} \psi$ est un sous-groupe ouvert de $I$. On peut donc trouver un élément $g \in I \cap \operatorname{Ker} \psi$ agissant sur $\mathbb{Q}_{p}\left(\mu_{p^{\infty}}\right)$ par un automorphisme d'ordre infini. Il suffit alors de choisir un caractère $\eta: \mathrm{W}_{\mathbb{Q}_{p}} \rightarrow \operatorname{Gal}\left(\mathbb{Q}_{p}\left(\mu_{p}\right) / \mathbb{Q}_{p}\right) \rightarrow \mathbb{C}^{*}$ tel que $\eta(g)^{2} \neq 1$. Par construction et injectivité de $\rho$, notons que l'extension de $\mathbb{Q}_{p}^{\text {ab }}$ fixée par le noyau la restriction de $\psi$ à $\mathrm{W}_{\mathbb{Q}_{p}} \cap \operatorname{Gal}\left(\overline{\mathbb{Q}}_{p} / \mathbb{Q}_{p}^{\mathrm{ab}}\right)$ est $K . \mathbb{Q}_{p}^{\mathrm{ab}}$.

Soit $\omega$ la représentation supercuspidale de $\mathrm{GL}_{n}\left(\mathbb{Q}_{p}\right)$ de $L$-paramètre $\psi$ donnée par la correspondance de Langlands locale $([\mathrm{HT}])$. Par construction, sa contragrédiente $\check{\omega}$ n'est isomorphe à aucune tordue de $\omega$ par un caractère non-ramifié. D'après le Théorème 3.2 il existe une représentation automorphe cuspidale irréductible $\Pi$ de $\mathrm{GL}_{2 n}\left(\mathbb{A}_{\mathbb{Q}}\right)$ ayant les propriétés suivantes :

i) $\check{\Pi} \simeq \Pi$,

ii) $\Pi$ est non ramifiée aux places finies différentes de $p$ et $\ell$,

iii) $\Pi_{p} \simeq I(\chi)$ pour un certain caractère non-ramifié $\chi: \mathbb{Q}_{p}^{*} \longrightarrow \mathbb{C}^{*}($ cf. 3.1 4.1 )

iv) $\Pi_{\infty}|\cdot|^{(2 n-1) / 2}$ est algébrique régulière et $\Pi_{\ell}$ est la représentation de Steinberg.

Sous les conditions i) et iv), les travaux de Kottwitz, Clozel et Harris-Taylor montrent qu'un choix quelconque de plongements $\ell$-adiques et complexes de $\overline{\mathbb{Q}}$ étant fait, on peut associer à $\Pi|\cdot|^{(2 n-1) / 2}$ une représentation continue

$$
\rho_{\Pi, \ell}: \mathrm{G}_{S} \longrightarrow \mathrm{GL}_{2 n}\left(\overline{\mathbb{Q}}_{\ell}\right)
$$


compatible à toutes les places finies $\neq \ell$ à la correspondance de Langlands locale « Frobenius semi-simplifiée » ([HT, [T, Thm. 3.6]).

Que cette représentation se factorise par $\mathrm{G}_{S}$ découle alors bien sûr de la condition ii). Le $L$-paramètre de $\Pi_{p} \simeq I(\chi)$ est la somme directe $\psi \otimes \chi \oplus \psi^{*} \otimes \chi^{-1}$ (cf. \$4.1). La compatibilité de $\rho_{\Pi, \ell} \mid W_{\mathbb{Q}_{p}}$ à $\Pi_{p}|\cdot|^{(2 n-1) / 2}$ assure en particulier que si $\mathbb{Q}(\Pi) \subset \mathbb{Q}_{S}$ est le sous-corps de $\overline{\mathbb{Q}}$ fixé par $\operatorname{Ker}\left(\rho_{\Pi, \ell}\right)$, alors

$$
\mathbb{Q}(\Pi) \cdot \mathbb{Q}_{p}^{\mathrm{ab}}=K \cdot \mathbb{Q}_{p}^{\mathrm{ab}} \subset E \cdot \mathbb{Q}_{p}^{\mathrm{ab}},
$$

ce qui conclut.

Puisqu'il existe une surjection continue de $\operatorname{Gal}\left(\overline{\mathbb{Q}}_{p} / \mathbb{Q}_{p}\right)$ vers $\widehat{\mathbb{Z}}$, le théorème cidessus admet le corollaire suivant en direction d'une question de J. Milne.

Corollaire 5.2. Si $|S| \geq 2$, alors pour tout entier $n \geq 1$ il existe un corps de nombres de degré divisible par $n$ qui est non ramifié hors de $S$.

Notons que ce corollaire admet par exemple le corollaire immédiat suivant : si $F$ est un corps local et $d \geq 1$, il n'existe pas de représentation continue injective $\mathrm{G}_{S} \rightarrow \mathrm{GL}_{d}(F)$.

Remarque 5.3. (i) Les résultats de cette section admettent des généralisations immédiates, avec les mêmes preuves, dans le cas où le corps de base $\mathbb{Q}$ est remplacé par un corps totalement réel $F$ quelconque. L'énoncé est alors le suivant : soit $S$ un ensemble fini non-vide de places finies de $F, v \in S$, et supposons que $S \backslash\{v\}$ contient toutes les places divisant un nombre premier $\ell$ donné; alors les applications naturelles

$$
\operatorname{Gal}\left(\overline{F_{v}} / F_{v}\right) \longrightarrow \operatorname{Gal}\left(F_{S} / F\right)
$$

sont injectives.

(ii) Une conséquence du théorème est que l'image de $\mathbb{Q}_{p} \cdot \mathbb{Q}_{S}$ dans $\overline{\mathbb{Q}}_{p}$ contient l'extension maximale non-ramifiée de $\mathbb{Q}_{p}$. Il est remarquable que par la méthode employée ici ce simple fait ne semble pas pouvoir s'obtenir à beaucoup moins de frais que le résultat tout entier. Le point est que nous ne pouvons pas prescrire totalement $\Pi_{p}$ pour les $\Pi$ que nous construisons mais seulement sa classe inertielle, et c'est bien évident : nous ne pouvons pas deviner à priori les nombres de Weil attachés au $L$-paramètre de $\Pi_{p}$ (à savoir le $\chi$ tel que $\left.\Pi_{p}=I(\chi)\right)$. Du coup, nous ne contrôlons jamais vraiment la partie non-ramifiée des corps de nombres que nous contruisons, bien que nous maîtrisions parfaitement leurs groupes d'inertie. Celle-là n'est récupérée qu'à la fin à cause de la structure du groupe de Galois local.

(iii) La question de savoir si le théorème vaut si $S=\{p\}$ semble nettement plus délicate (voir [Ch, §4.2]). Il est cependant tentant de conjecturer que le théorème est aussi vrai dans ce cas.

\section{RÉFÉRENCES}

[A1] J. Arthur, The invariant trace formula I. Local theory, J. Amer. Math. Soc. 1 (1988), 323-383. MR928262 (89e:22029)

[A2] J. Arthur, The invariant trace formula II. Global theory, J. Amer. Math. Soc. 1 (1988), 501-554. MR939691 (89j:22039)

[A3] J. Arthur, The local behaviour of weighted orbital integrals, Duke Math. J. 56 (1988), 223-293. MR932848 (89h:22036) 
[A4] J. Arthur, An introduction to the trace formula, in Harmonic Analysis, the trace formula and Shimura varieties, A.M.S., Clay Math. Institute (2005). MR2192011 (2007d:11058)

[AC] J. Arthur et L. Clozel, Simple algebras, base change, and the advanced theory of the trace formula, Ann. of Math. Studies 120 (1989). MR1007299 (90m:22041)

[Be] J.-N. Bernstein (rédigé par P. Deligne), Le « centre » de Bernstein, in Bernstein, Deligne, Kazhdan, Vignéras, Représentations des groupes réductifs sur un corps local, Hermann, Paris (1984). MR771671 (86e:22028)

[BLS] A. Borel, J.-P. Labesse et J. Schwermer, On the cuspidal cohomology of S-arithmetic subgroups of reductive groups over number fields, Compositio Math. 102 (1996), 1-40. MR1394519 (97j:11026)

[BW] A. Borel et N. Wallach, Continuous cohomology, discrete subgroups, and representations of reductive groups, Annals of Mathematics Studies 94 (1980). MR.554917 (83c:22018)

[Bou] A. Bouaziz, Sur les caractères des groupes de Lie réductifs non connexes, J. Funct. Analysis 70 (1987), 1-79. MR870753 (89c:22020)

[BT] F. Bruhat et J. Tits, Groupes réductifs sur un corps local : I. Données radicielles valuées, Pub. Math. IHES 41 (1972), 5-251. MR0327923 (48:6265)

[Cas] W. Casselman, A new nonunitarity argument for p-adic representations, J. Fac. Sci. Univ. Tokyo 28 (1981), 907-928. MR656064 (84e:22018)

[Ch] G. Chenevier, On number fields with given ramification, Compositio Math. 143 (2007), 1359-1373. MR 2371372

[Clo1] L. Clozel, Théorème d'Atiyah-Bott pour les variétés $\mathfrak{p}$-adiques et caractères des groupes réductifs, Harmonic analysis on Lie groups and symmetric spaces (Kleebach, 1983), Mém. Soc. Math. France 15 (1984), 39-64. MR789080 (87b:22034)

[Clo2] L. Clozel, Motifs et formes automorphes : Applications du principe de fonctorialité, in Automorphic forms, Shimura varieties, and L-functions, Clozel, Milne eds. vol. I, Perspectives in Math. 10, Academic Press (1990), 77-159. MR1044819 (91k:11042)

[CloD] L. Clozel et P. Delorme, Le théorème de Paley-Wiener invariant pour les groupes de Lie réductifs II, Ann. Sci. École Norm. Sup. 23 (1990), 193-228. MR1046496 (91g:22013)

[HT] M. Harris et R. Taylor, The geometry and cohomology of some simple Shimura varieties, Annals of Math. Studies 151 (2001). MR.1876802 (2002m:11050)

[He1] G. Henniart, La conjecture de Langlands locale pour GL(3), Mém. Soc. Math. France, no. 11-12 (1984). MR743063 (86g:11070)

[He2] G. Henniart, Correspondance de Langlands et fonctions $L$ des carrés extérieur et symétrique. Prépublications Mathématiques de l'IHES (2003).

[Hi] A. Hitta, On the continuous (co)homology of locally profinite groups and the Künneth theorem, J. Algebra 163 (1994), 481-494. MR1262715 (94m:22004)

[K1] R. Kottwitz, Tamagawa numbers, Ann. of Math. (2) 127 (1988), 629-646. MR942522 (90e:11075)

[K2] R. Kottwitz, Rational conjugacy classes in reductive groups, Duke Math. J. 49 (1982), 785-806. MR683003 (84k:20020)

[KR] R. Kottwitz et J. Rogawski, The distributions in the invariant trace formula are supported on characters, Canad. J. Math. 52 (2000), 804-814. MR.1767403 (2001e:11117)

[KS] R. Kottwitz et D. Shelstad, Foundations of twisted endoscopy, Astérisque 255 (1999). MR.1687096 (2000k:22024)

[La1] J.-P. Labesse, Cohomologie, stabilisation et changement de base, Astérisque 257 (1999). MR.1695940(2001k:22040)

[La2] J.-P. Labesse, Pseudo-coefficients très cuspidaux et K-théorie, Math. Ann. 291 (1991), 607-616. MR.1135534 (93b:22026)

[Lg] R. P. Langlands, Automorphic representations, Shimura varieties, and motives. Ein Märchen, in Automorphic Forms, Representations, and L-Functions, Proc. Sympos. Pure Math. 33 vol. II, A.M.S., Providence (1977), 205-246. MR546619 (83f:12010)

[M] P. Mezo, Twisted trace Paley-Wiener theorems for special and general linear groups, Compositio Math. 140 (2004), 205-227. MR2004130(2004e:22010) 
[NSW] J. Neukirch, A. Schmidt et K. Wingberg, Cohomology of Number Fields, Springer-Verlag, Grundlehren 323 (2000). MR:1737196 (2000j:11168)

[PR] D. Prasad et D. Ramakrishnan, On self-dual representations of division algebras over local fields. Prépublication.

[Ro] J. Rogawski, Trace Paley-Wiener theorem in the twisted case, Trans. A.M.S. 309 (1988), 215-229. MR957068 (89k:22035)

[S1] J.-P. Serre, Cohomologie des groupes discrets, Prospects in Mathematics, Ann. of Math. Studies 70 (1971), 77-169. MR0385006 (52:5876)

[S2] J.-P. Serre, Répartition asymptotique des valeurs propres de l'opérateur de Hecke $T_{p}$, J. Amer. Math. Soc. 10 (1997), 75-102. MR1396897(97h:11048)

[Sh1] F. Shahidi, A proof of Langlands' conjecture on Plancherel measures; complementary series for p-adic groups, Ann. of Math. (2) 132 (1990), 273-330. MR.1070599(91m:11095)

[Sh2] F. Shahidi, Twisted endoscopy and reducibility of induced representations for $p$-adic groups, Duke Math. J. 66 (1992), 1-41. MR1159430 (93b:22034)

[She] D. Shelstad, Characters and inner forms of a quasi-split group over $\mathbb{R}$, Compositio Math. 39 (1979), 11-45. MR539000 (80m:22023)

[T] R. Taylor, Galois representations, Annales de la Faculté des Sciences de Toulouse 13 (2004), 73-119. MR2060030 (2005a:11071)

[TY] R. Taylor et T. Yoshida, Compatibility of local and global Langlands correspondences, J. Amer. Math. Soc. 20 (2007), 467-493. MR2276777 (2007k:11193)

[Ti] J. Tits, Reductive groups over local fields, in Automorphic forms, representations and Lfunctions, Proc. Sympos. Pure Math. 33 vol. I, A.M.S., Corvallis (1977), 29-69. MR546588 (80h:20064)

[V] D. Vogan, Unitarizability of certain series of representations, Ann. of Math. (2) 120 (1984), 141-187. MR750719 (86h:22028)

[W1] J.-L. Waldspurger, Le groupe GL(n) tordu, sur un corps p-adique, partie I, Duke Math. J. 137 (2007), 185-234. MR2309147

[W2] J.-L. Waldspurger, Le groupe $\mathrm{GL}(n)$ tordu, sur un corps p-adique, partie II, Duke Math. J. 137 (2007), 235-336. MR.2309148

Laboratoire Analyse, Géométrie et Applications, UMR 7539, Institut Galilée, Université Paris 13, 99 Av. J-B. Clément, 93430 Villetaneuse, France

Centre d’Orsay Mathematique, Université Paris Xi, Batiment 425, 91405 Orsay Cedex FRANCE 